Utah State University

DigitalCommons@USU

\title{
$5-2013$
}

\section{The Psychological and Physiological Effects of Using a Therapy Dog in Mindfulness Training}

Courtney L. Henry

Utah State University

Follow this and additional works at: https://digitalcommons.usu.edu/etd

Part of the Clinical Psychology Commons

\section{Recommended Citation}

Henry, Courtney L., "The Psychological and Physiological Effects of Using a Therapy Dog in Mindfulness Training" (2013). All Graduate Theses and Dissertations. 1957.

https://digitalcommons.usu.edu/etd/1957

This Dissertation is brought to you for free and open access by the Graduate Studies at DigitalCommons@USU. It has been accepted for inclusion in All Graduate Theses and Dissertations by an authorized administrator of DigitalCommons@USU. For more information, please contact digitalcommons@usu.edu.

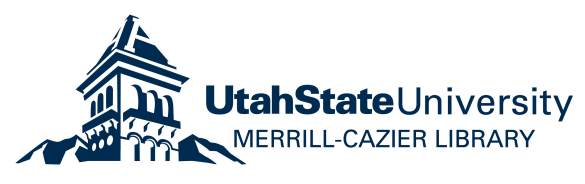




\title{
THE PSYCHOLOGICAL AND PHYSIOLOGICAL EFFECTS OF USING A THERAPY DOG IN MINDFULNESS TRAINING
}

\author{
by \\ Courtney L. Henry \\ A dissertation submitted in partial fulfillment \\ of the requirements for the degree \\ of \\ DOCTOR OF PHILOSOPHY \\ in \\ Psychology
}

Approved:

Susan Crowley, Ph.D.

Major Professor

Michael Twohig, Ph.D.

Committee Member

Christopher Johnson, Ph.D.

Committee Member
Scott DeBerard, Ph.D.

Committee Member

Edward Heath, Ph.D.

Committee Member

UTAH STATE UNIVERSITY

Logan, Utah

Mark McLellan, Ph.D.

Vice President for Research and

Dean of the School of Graduate Studies 
Copyright (C) Courtney Henry 2013

All Rights Reserved 


\author{
ABSTRACT \\ The Psychological and Physiological Effects of Using a Therapy Dog \\ in Mindfulness Training \\ by \\ Courtney L. Henry, Doctor of Philosophy \\ Utah State University, 2013
}

Major Professor: Dr. Susan L. Crowley

Department: Psychology

Research from various fields has demonstrated the benefits of human-animal interaction for physical and mental health. Recently, animal-assisted therapy (AAT) has become increasingly popular in a variety of healthcare settings - including inpatient mental health care facilities. However, there is limited research investigating the efficacy of AAT in outpatient sites. In addition, the impact of animals as an adjunct to psychotherapy treatment remains mostly uninvestigated. Therefore, it is necessary to empirically explore what therapy animals may contribute to specific treatment interventions with specific populations.

The present study was a randomized control trial examining the psychological and physiological effects of adding AAT to a modified mindfulness-based stress reduction program (MBSR) for clients experiencing psychological distress, including anxious and depressive symptoms. It was hypothesized that AAT would be particularly complementary to mindfulness-based interventions because the therapy dog would 
provide a focus for attention to the current experience and exemplify acceptance and "being," enabling the understanding and practice of the main aspects of mindfulness. Subjects $(N=21)$ were randomly assigned to the MBSR or MBSR + AAT group and then completed an intervention consisting of six 50-minute individual therapy sessions. Each session included didactic and experiential components modified for delivery with or without a certified therapy dog.

State and trait mindfulness, state and trait anxiety, psychological distress, blood pressure, and heart rate were assessed at each session. Results indicate that all participants experienced fewer anxiety and depressive symptoms, decreased psychological distress, and increased mindfulness skills from pre- to posttreatment. Additionally, state anxiety, blood pressure, and heart rate decreased within sessions. No significant difference was found between the control and experimental groups, indicating that interaction with a therapy dog had no impact on symptom reduction, skill acquisition, or client satisfaction in the current study. Future studies need to increase methodological rigor by including multiple therapist/dog teams and increasing sample size. Moreover, researchers must examine more thoroughly the role the dog might have in altering the social environment, such as reducing stigma surrounding mental health services and enhancing the therapeutic alliance. 
PUBLIC ABSTRACT

The Psychological and Physiological Effects of Using a Therapy Dog

in Mindfulness Training

by

Courtney L. Henry, Doctor of Philosophy

Utah State University, 2013

This study examined the psychological and physiological effects of adding animal-assisted therapy to a modified mindfulness-based stress reduction program (MBSR) for clients experiencing psychological distress, including anxious and depressive symptoms. State and trait mindfulness, state and trait anxiety, psychological distress, blood pressure, and heart rate were collected during each session of the intervention from college students recruited at Utah State University. Results support that participants experienced less anxiety and depressive symptoms and increased mindfulness skills from pre- to posttreatment. No significant difference was found between the control and experimental groups, indicating that that interaction with a therapy dog had no impact on symptom reduction, skill acquisition, or client satisfaction. Treatment occurred at the USU Psychology Community Clinic. The six 50-minute session intervention was provided by a graduate student therapist and certified therapy dog under the supervision of a licensed psychologist. Participants who completed the intervention were each given a $\$ 20$ electronic gift card to Amazon.com or iTunes. No costs to the participants were anticipated beyond the time spent participating. Benefits of the study include adding to the existing knowledge base regarding the effects of animal-assisted therapy on psychological and physiological functioning in humans. 


\section{ACKNOWLEDGMENTS}

I would like to thank my committee members, Drs. Susan Crowley, Scott DeBerard, Michael Twohig, Christopher Johnson, and Edward Heath, for their assistance throughout my research process. I would especially like to thank Dr. Crowley for her ongoing mentorship and guidance. I also thank the staff at Utah State University's Counseling and Psychological Services for their caring encouragement and help in recruiting.

I give thanks to my mother, who has endlessly supported me throughout my graduate studies and beyond; my father and his wife, who could not be more proud of me; and my grandmothers, who continue to inspire me. I also thank my friends and colleagues, especially Christine and Mac, for providing both motivation and distraction. I thank the Calhoun family for being my home away from home. I would like to thank Francisco for teaching me more about love and life than a classroom ever could. Finally, I would like to thank my animals, Moxie, Lucky, Kismet, and Karma, for being my furry therapists and keeping me in the moment, especially Lucky who made this research possible.

Courtney L. Henry 


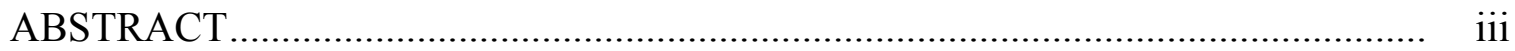

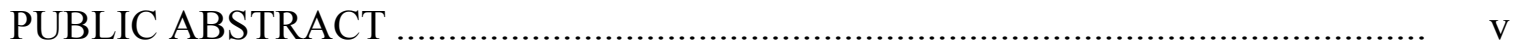

ACKNOWLEDGMENTS ……………………….......................................... vi

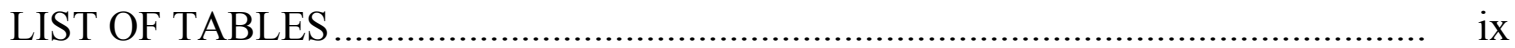

\section{CHAPTER}

I. INTRODUCTION .......................................................................... 1

II. REVIEW OF THE LITERATURE …………………………………..... 5

Animal-Assisted Therapy ..................................................................... 5

Mindfulness.................................................................................. 22

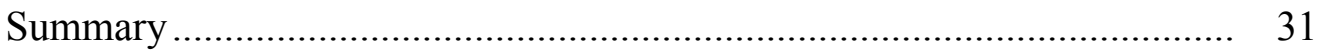

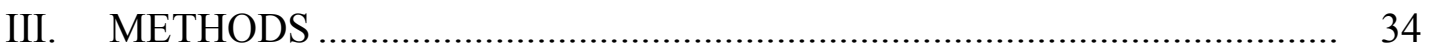

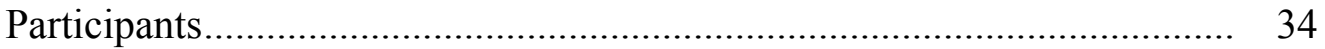

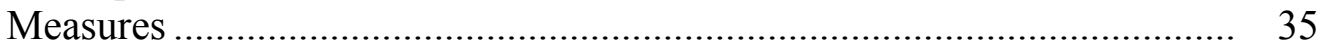

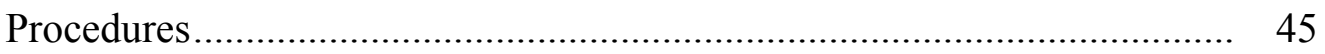

Modified MBSR Intervention .................................................................. 47

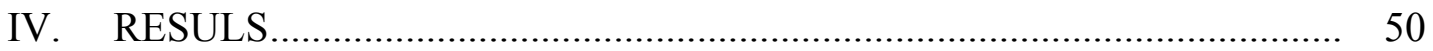

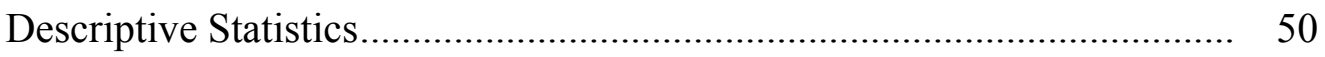

Development of Mindfulness Skills......................................................... 51

Reduction in Anxiety During Treatment ………………....................... 55

Reduction in Psychological Distress..................................................... 58

Client Satisfaction and Engagement ....................................................... 61

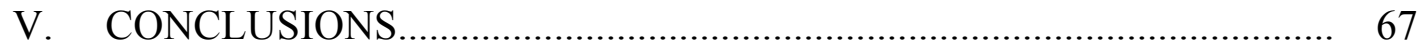

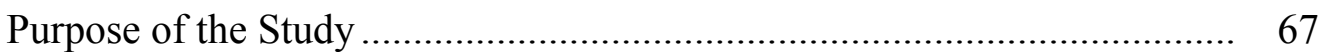

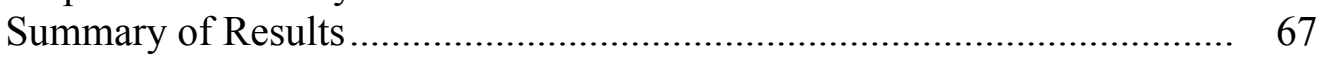

Addressing Gaps in the Literature ........................................................... 70

Limitations and Future Research ........................................................... 73

Recommendations for Clinical Practice..................................................... 76 


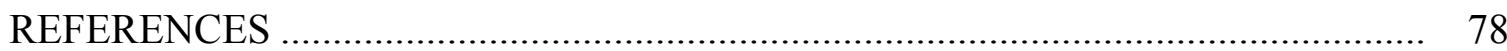

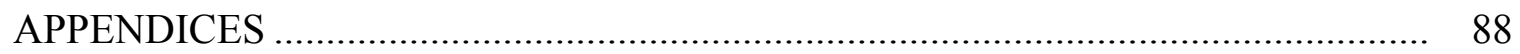

Appendix A: Intervention Protocol ….................................................. 89

Appendix B: Measures .......................................................................... 99

Appendix C: Descriptive Statistics and Correlation Tables .................... 110

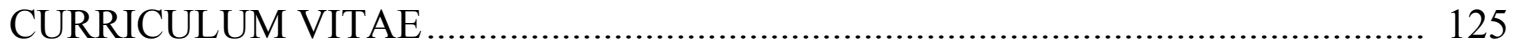




\section{LIST OF TABLES}

Table Page

1. Demographic Information........................................................................ 36

2. Information for Noncompleters ........................................................ 36

3. Comparison of Pretreatment Scores between Groups for the OQ-45, BDI-II, BAI, A-Trait, FFMQ, and PHLMS ................................................ 51

4. Comparison of Pre- and Posttreatment Trait Mindfulness ........................... 53

5. Comparison of State Mindfulness by Session ........................................... 54

6. Pairwise Comparisons of State Mindfulness Between Sessions..................... 54

7. Comparison of Change in State Anxiety by Session .................................. 56

8. Pairwise Comparison of State Anxiety Change Between Sessions ................. 56

9. Comparison of Change in Systolic Blood Pressure by Session ...................... 57

10. Comparison of Change in Diastolic Blood Pressure by Session ..................... 58

11. Comparison of Change in Heart Rate by Session ....................................... 59

12. Comparison of Pre- and Posttreatment Distress ........................................ 60

13. Comparison of Psychological Distress by Session .................................. 61

14. Pairwise Comparisons of Psychological Distress Between Sessions ............... 62

15. Comparison of Change in Mindfulness and Engagement by Session .............. 63

16. Pairwise Comparison of Mindfulness and Engagement Between Sessions ...... 63

17. Comparison of Change in Positive Anticipation and Practice by Session........ 64

18. Comparison of Client Satisfaction Between Groups ................................. 65

A1. Outline of Session Timing and Materials .............................................. 91

A2. Modified Mindfulness-Based Stress Reduction Treatment Components ......... 92 
C1. Descriptive Statistics for the BDI-II, BAI, STAI-Trait, FFMQ, and PHLMS .. 111

C2. Descriptive Statistics for the OQ-45 ...................................................... 112

C3. Descriptive Statistics for the A-State ......................................................... 113

C4. Descriptive Statistics for the TMS ........................................................ 114

C5. Descriptive Statistics for Session Engagement Questionnaire ....................... 115

C6. Descriptive Statistics for Client Satisfaction Questionnaire .......................... 118

C7. Descriptive Statistics for Blood Pressure and Heart Rate............................... 119

C8. Pearson Correlations Between Measures of Control (MBSR) Group .............. 122

C9. Pearson Correlations Between Measures of Experimental (MBSR+AAT)

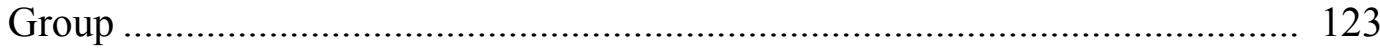

C10. Pearson Correlations Between Measures of Total Sample ............................. 124 


\section{CHAPTER I \\ INTRODUCTION}

Humans today spend less time interacting with animals than in previous generations (Katcher \& Beck, 1987). Current research indicates that this separation from nature produces less healthy lifestyles for people (Katcher \& Beck, 1987; Stilgoe, 2001); and further, research from a variety of fields has demonstrated the benefits of humananimal interaction for physical and mental health (Friedmann \& Thomas, 1995; Katcher \& Beck, 1983; E. O. Wilson, 1984). Over time, animal-assisted therapy (AAT) and animal-assisted activities (AAA) have become increasingly popular in a variety of healthcare settings, including hospitals, nursing homes, rehabilitation centers, residential care sites, and outpatient mental care facilities (Delta Society, 2010). Therefore, as the use of animals in therapy increases, it is necessary to empirically explore what therapy animals may contribute to human mental health.

Although animals have been involved in therapy activities for decades, there is relatively little empirical evidence to support the theorized mental and physical health benefits in an outpatient clinical population. Previous studies have focused mainly on elderly populations and have relied heavily on self-report data. Therefore, the challenge in the field is to investigate using more rigorous methodology (C. C. Wilson, 2006), particularly studying a more generalizable population in a controlled research design with multiple data sources. The current study addressed these gaps in the previous literature by using a randomized control trial to examine the use of AAT with a college population, collecting both self-report and physiological data. 
Based on the literature to date, there is some empirical evidence to support that human-animal interaction decreases blood pressure (Friedmann, Thomas, \& Eddy, 2000), lowers heart rate (DeSchriver \& Riddick, 1990), reduces anxiety (C. C. Wilson, 1991), lessens depression (Souter \& Miller, 2007), and enhances the social environment (Wells $\&$ Perrine, 2001). Theorists propose that these and other benefits may arise from the ability of positive human-animal interaction to affect humans through multiple pathways, including physical, psychological, and social (Friedmann \& Tsai, 2006). Moreover, recent studies indicate that the use of a companion animal in therapy may produce a wide array of similar benefits for various clinical populations (Chandler, 2005; C. Wilson \& Turner, 1998).

Given the reported benefits of human-animal interaction, it is possible that AAT might be a beneficial complementary treatment for people experiencing psychological distress, such as anxious and depressive symptoms. Prevalence rates of clinical depression and anxiety disorders have increased dramatically during the last half-century in the U.S. (Klerman \& Weissman, 1989; Compton, Conway, Stinson, \& Grant, 2006); and psychologists are constantly striving to improve intervention techniques and enhance treatment outcomes for people who suffer from these symptoms. Therefore, research is needed to investigate if adding AAT to established psychological interventions increases treatment efficacy.

Although there are many strategies available to decrease psychological distress, one technique frequently used to address anxiety and depressive symptoms is the teaching of mindfulness practices. Mindfulness is defined as an awareness of one's 
present internal and external experiences with acceptance and kindness (Kabat-Zinn, 1990). Mindfulness-based therapy is, therefore, any treatment that includes mindfulness practice as a primary component (Shapiro \& Carlson, 2009). Mindfulness-based stress reduction (MBSR; Kabat-Zinn, 1990) and mindfulness-based cognitive therapy (MBCT; Segal, Williams, \& Teasdale, 2002) are two such interventions that emphasize nonjudgmental awareness of the present moment to alleviate psychological distress. Additionally, several interventions, such as acceptance and commitment therapy (ACT) and dialectical behavior therapy (DBT), employ mindfulness techniques as one component of the intervention package.

There is a strong body of research evidence supporting the efficacy of mindfulness in treating a variety of clinical concerns (Baer, 2003; Bohlmeijer, Prenger, Taal, \& Cuijpers, 2010; Grossman, Niemann, Schmidt, \& Walach, 2004; Hofmann, Sawyer, Witt, \& Oh, 2010; Ledesma \& Kumano, 2008). MBSR was first used to treat chronic pain and has more recently been used to treat emotional pain, such as depression. In addition, some mindfulness-based interventions have been shown to be empirically supported for specific diagnoses; the Society of Clinical Psychology (American Psychological Association [APA], Division 12) recognizes acceptance and commitment therapy and dialectical behavior therapy as empirically supported treatments for depression and borderline personality disorder, respectively (APA, 2010). Because the teaching and practice of mindfulness is frequently used in treatment, it seems worthwhile to research ways in which the therapeutic efficacy of mindfulness might be increased.

Based on current research findings, it is hypothesized that AAT will increase the 
efficacy of mindfulness training at decreasing psychological distress. Additionally, animals provide a focus of attention and offer unconditional positive regard. Because of these innate qualities of animals, it is hypothesized that AAT will particularly complement the aims of mindfulness practice, including giving attention to the present moment and embracing a nonjudgmental attitude. This study will attempt to address a challenge put forth by C. C. Wilson (2006), and Fine and Mio (2006) to produce methodologically rigorous and empirically sound research for the field of AAT by examining the psychological and physiological effects of adding AAT to a modified MBSR program for clients with anxious and depressive symptoms. 


\section{CHAPTER II}

\section{REVIEW OF THE LITERATURE}

The present literature review will attempt to summarize the available information on the effects of AAT on psychological distress and physiological arousal. AAT will be defined; and a brief history of the development of the intervention and theoretical frameworks will be provided. Empirical evidence regarding the efficacy of AAT in decreasing psychological distress such as anxious and depressive symptoms, and reducing heart rate and blood pressure will be examined and gaps in the literature will be identified. Next, the use of mindfulness practice in the treatment of psychological distress and its effects on physiological arousal will be reviewed; and areas in need of more research will be acknowledged. Lastly, a brief summary of pertinent conclusions from the literature and implications for the present research proposal will be offered.

\section{Animal-Assisted Therapy}

Given the long record of human-animal interaction, it is interesting to note that never before in history have humans spent such little time physically engaging with the natural environment, including plants and animals, as they do now (Katcher \& Beck, 1987). With the advancement of technology and cultural change, people no longer need to rely on direct contact with animals to provide food or protection. Yet, animals are still in many homes; in 2006, a national survey found that there are approximately 72 million pet dogs and 82 million pet cats in the U.S. (American Veterinary Medical Association [AVMA], 2007). Researchers have found that avoidance of loneliness and motivation to 
remain physically active are the top two reasons given by pet owners for having animals (Staats, Wallace, \& Anderson, 2008); and nearly half (49.7\%) of pet owners consider their pets to be family members (AVMA, 2007). Research indicates that it may be attributes of companionship that maintain the human-animal bond (Serpell, 1996) and provide various physical and mental health benefits (Katcher \& Beck, 1983).

\section{A Brief History of Human-Animal Interaction}

Animals have always had a part in the human experience. What that role is, however, has changed dramatically throughout the ages. Early in history, animals were frequently used as a source of food. Cave drawings from 10,000 years ago depict people

chasing and spearing bison; and big game hunting expeditions became tradition in many cultures (Mithen, 1999). Yet in some of these same societies, animals served as deities and were symbols of various strengths. For example, ancient Egyptian gods and goddesses were represented in animal forms (Waldau \& Patton, 2006). Furthermore, people and animals found mutual benefits from interactions that did not end in death or idolization. It is theorized that wild dogs became domesticated after forming working relationships with humans, including protectors, hunting companions, and later, herders. Canines provided help and humans offered shelter and food (Omori \& Hasegawa, 2009). Early humans lived with these wild animals, learning about their abilities, their habitats, their family groups, and, in general, their places in the ecosystem. Often at the same time, people learned to kill animals, fear them, revere them, control them, need them, avoid them, and respect them. It is this complicated pattern of antithetical relationships that 
characterizes many of the interactions between animals and people.

Animal companionship has been observed to have socializing influences on people since the late $17^{\text {th }}$ century, when the age of enlightenment brought about changes in attitudes towards animals. People became less anthropocentric and, with the movement of people out of rural areas, more likely to keep pets (Salisbury, 2010; Serpell, 1996). At this same time, philosophers on the human condition wrote of the benefits of humananimal interaction on healthy child development. John Locke (1693) stated that animal care aided in the development of empathy and moral reasoning in young children; and, following the beliefs of Thomas Hobbes, other philosophers believed that children could learn to control their own innate savage tendencies through training animals (Myers, 1998). Using the same reasoning, animals were frequently permitted in mental institutions throughout Europe by the $19^{\text {th }}$ century. One example of the socializing use of animals occurred at the York Retreat in England, where the founder, William Tuke, provided small domesticated animals with which inmates could interact and develop empathetic feelings (McCulloch, 1983). Yet by the early $20^{\text {th }}$ century, domesticated animals could rarely be found in institutional care due to the advent of scientific medicine (Allderidge, 1991).

Meanwhile, the first scholarly papers on the potential psychological value of animals appeared also in the early $20^{\text {th }}$ century and were primarily focused on the ways in which children benefited from having pets beyond learning self-control. For example, Bossard (1944), a psychiatrist, hypothesized that pets increased empathy, self-esteem, and communication skills in children. However, these writings contained little theoretical 
support; and it was not until the contributions of Boris Levinson in the early 1970s that the experience of "pet therapy" received attention from researchers and practitioners (Serpell, 1996). Similar to Freud's conceptualization of the "id" as the basic animal-like instinct of the human psyche, Levinson believed that animals represent humans' irrational selves. According to Levinson (1972), people's increasing sense of alienation is due to a lack of connection with their own unconscious animal natures, like the unhealthy repression of beastly impulses into the subconscious (Serpell, 2000). To heal this disconnect, humans must establish positive relationships with real animals, providing companionship on the path to emotional well-being (Levinson, 1969). Thus, Levinson stated that because animals have played a key role in providing humans with membership in the natural world throughout evolution, the human-animal relationship has remained imperative to psychological health.

While emphasizing the importance of pets as companions, Levinson (1969) also wrote of the benefits of animals as "co-therapists" and cited the reasoning of contemporary psychotherapists who used dogs, cats, or other small animals in treatment. Although Levinson's work recognized psychological links between animals and health, his writing did little to provide empirical support for the benefit of animal companionship. In 1980, a landmark study by Friedmann, Katcher, Lynch, and Thomas (1980) sparked an examination of the therapeutic effects of pet ownership by the medical community (Serpell, 2000). The study investigated the effects of social isolation on the survival rates of people who had suffered heart attacks. Data on mood and social conditions were collected during recovery and survival after one year was recorded. As 
hypothesized, results concluded that certain types of social contact were important predictors of survival; however, it was also determined that pet-owners had significantly better chances of survival than nonowners (Friedmann et al., 1980).

Multiple physical health-related studies followed. Some focused on the measurement of physiological responses to animals. For example, Friedmann (1983) found that children's blood pressures were lower in the presence of a friendly dog while resting and reading compared to without a dog. Other studies attempted to examine the role of companion animals as social support, assuming a bio-psycho-social perspective. Garrity, Stallones, Marx, and Johnson (1989) examined pet ownership, emotional distress, social support, and illness symptoms in a sample of U.S. elderly; although results showed no correlation between illness and pet ownership, researchers did find that pet attachment may be related to decreased emotional distress. (For a review of similar research, see Garrity \& Stallones, 1998.) Increased research on human-animal interactions led to the development of more theories regarding the mechanisms underlying the benefits of companion animals.

Presently, there are a growing number of organizations throughout the world supporting research on human-animal interaction or the practice of AAT. For example, the International Organization of Human Animal Interaction Organizations (IOHAIO) has 35 current members, including associations for AAT, pet nutrition, animal welfare, and research (IOHAIO, 2013). Since the 1970s, organizations have developed in the United States that focus on providing animal-assisted services, as well as training service providers and developing service standards. Therapy Dogs International (TDI) was 
founded in 1976 and continues to be the largest therapy dog organization in the United States. In 2003, over 14,000 dogs and 10,500 handlers were approved by and registered with TDI to provide services (TDI, 2010). Another similar organization is the Delta Society, which promotes the therapeutic work of dogs and other domesticated and farm animals in a variety of institutional settings; Delta Society provides training, evaluation, and certification for animal-handler teams (Delta Society, 2010). The North American Riding for the Handicapped Association (NARHA, 2010), established in 1969, has evolved to incorporate mental health interventions through the use of equine-assisted physical therapy in its special interest sector, the Equine Facilitated Mental Health Association (EFMHA). Furthermore, there are over a dozen universities in the United States that currently have professional research, training, service centers, or programs in AAT or human-animal interaction. Although still not widely accepted in the medical or mental health fields, AAT has come a long way in establishing its potential value to mental health professionals and the clients they serve.

\section{Mechanisms of Therapeutic Benefits and Theoretical Frameworks}

Currently, the field of AAT lacks an agreed-upon theoretical framework to explain why animals may provide humans with therapeutic benefits. Given the variety of findings from research that explores the effects of human-animal interaction, it is not surprising that there has been a variety of potential mechanisms of change proposed by researchers and clinicians. According to Kruger and Serpell (2006), most of the theories found in literature pertain to the various intrinsic qualities specific to animals that 
contribute to therapy. From this view, the simple presence of an animal in therapy provides opportunities that may not arise in its absence, including reduction of anxiety, social mediation, and emotional attachment. Other theories support the idea that animals can be used as tools to affect changes in thoughts and behaviors related to self-efficacy and responsibility. Many equine-assisted therapy programs, as well as programs that include animal training or caretaking, are based on cognitive and social cognitive theories and emphasize the working relationship between human and animal (Kruger \& Serpell, 2006). The interaction is believed to facilitate the learning of various skills sets and provide immediate feedback about behaviors. Given the way in which a dog will be incorporated into therapy in the currently proposed study, only theoretical frameworks supporting the inherent attributes of animals as mechanisms of change will be further examined in this literature review. Cognitive theories that view the animal as a tool will not be addressed.

First, the presence of an animal is often reported to have antianxiolytic effects on people (see previously reviewed studies), decreasing arousal through various possible mechanisms. One theory used to explain the anxiety-reducing effect of animals is the biophilia hypothesis by E. O. Wilson (1984). This theory states that humans have an innate tendency to be attracted by and attend to other living things, including animals and plants. From an evolutionary standpoint, the chance of survival increases for humans as they pay attention to and learn about their environmental surroundings; therefore, humans may have an increased awareness of, and possibly interest in, animals because of their evolutionary interdependence. Furthermore, evidence suggests that various stimuli that 
divert and concentrate attention, including swimming fish, log fires, and kinetic sculptures, can produce a calming effect on people (Katcher, Friedmann, Beck, \& Lynch, 1983; Katcher, Segal, \& Beck, 1984). Therefore, animals in therapy can provide a focus for attention while also decreasing arousal. In other words, animals supply practitioners with "a tool that can simultaneously engage and relax the patient" (Kruger \& Serpell, 2006). It is important to note that the biophilia hypothesis is difficult to test in its general form, as its definition encompasses a variety of living things. Furthermore, attitudes and practices toward animals greatly differ by culture, making it difficult to examine biological influences without much cross-cultural research (A. M. Beck \& Katcher, 2003).

Another explanation for the anxiety-reducing effect of animals used in therapy is found in learning theory, as proposed by Brickel (1985). According to learning theory, pleasurable activities are self-reinforcing, increasing the likelihood that a person will engage in them again. Unpleasant stimuli, on the other hand, can result in avoidance, which is then negatively reinforced. Brickel suggested that animals provide a buffering effect against anxiety-inducing stimuli encountered in therapy, such as engaging in painful discussions with a therapist, by providing a distraction during continued exposure to therapy. Therapy is then paired with nonaversive consequences, resulting in the eventual extinction of anxiety. Examined from a different perspective, learning theory can provide support for the idea that the presence of an animal offers positive reinforcement for attending therapy to clients that enjoy interacting with animals. This repeated pairing of a positively reinforcing stimulus (the animal) with an aversive 
stimulus (such as an anxiety-provoking discussion) can also lead to the extinction of anxiety associated with therapy. However, learning theory still assumes that an animal provides a stimulus that uniquely attracts a person's attention.

Another way that researchers are exploring the calming effect of animals is through human physiology. Some studies have indicated that stress-related activity by the body is reduced when animals are present; and theorists are using these results to support subjective reports of decreased anxiety found in other studies. However, the results of physiological studies have been mixed (Friedmann \& Tsai, 2006) and there is still no agreed-upon hypothesis explaining why animals may be related to changes in physiology and a subsequent experience of decreased arousal in humans. Furthermore, it is possible that animals have a calming effect on humans due to a combination of mechanisms explained by multiple theories. Unfortunately, there is currently not enough evidence to pinpoint any one explanation; however, there does seem to be enough support to state that animals in certain contexts can reduce anxiety for certain people.

Second, animals have been observed to provide a form of social mediation between humans. It is suggested that animals may facilitate the building of rapport between client and therapist by providing a neutral subject on which to converse and may reduce anxiety during initial therapy sessions, expediting the process (Levinson, 1969). Fine (2006) described a case study in which a child with selective mutism was willing to initially speak only to and about a therapy dog; and she cited researchers and practitioners who have drawn similar conclusions based on their work. Furthermore, it has been noted that animals in psychotherapy can provide a catalyst for experiencing various emotions 
(Fine, 2006). Animals can act as mood regulators, easing sorrow through comforting interactions as well as inducing laughter through humorous behaviors. This regulatory aspect of AAT is theorized to provide a stabilizing effect, allowing clients to experience a fuller range of emotions in the presence an animal. Based in psychoanalytic theory, some practitioners describe clients that have been able to discuss emotionally difficult topics by projecting them onto an animal (Serpell, 2000). Moreover, animals can display affect through behaviors that may not be professionally appropriate for the human therapist, such as physical touch. In these ways, the animal may act as an extension of the therapist, facilitating the relationship-forming process.

It has also been theorized that animals may alter perceptions of social desirability, which may mediate social interactions between humans. Studies have demonstrated that college students perceive people with a dog as more relaxed, happier, friendlier, and less threatening than people without a dog (Rossbach \& Wilson, 1992; Wells \& Perrine, 2001). Other studies have shown that positive social interactions with strangers are significantly increased when people in wheelchairs are seen with service dogs as compared to without (Eddy, Hart, \& Boltz, 2001; Mader, Hart, \& Bergin, 1989). Overall, there is evidence that supports the ability of animals to mediate social interactions between people, which may enhance the therapeutic alliance between client and practitioner. As noted by Kruger and Serpell (2006), this "social lubricant" feature of AAT may be of particular value, as treatment outcomes, compliance, and retention may be highly correlated to the working alliance (Horvath \& Bedi, 2002; Howgego, Yellowlees, Owen, Meldrum, \& Dark, 2003). 
Last, a third way in which animals are theorized to provide therapeutic benefits to humans is by providing a nonjudgmental entity for emotional attachment. According to attachment theory as put forward by Bowlby and Harlow, humans have a need for loving social interaction that goes beyond feeding and basic physical care (van der Horst, LeRoy, \& van der Veer, 2008). Observable behaviors such as caring touch and close physical proximity, which happen between children and their attachment figures, also occur between humans and animals (Triebenbacher, 1998); and, as noted previously, many people report avoidance of loneliness as a primary reason for having a pet. These facts support the idea that humans can form strong attachments to animals and animals can fulfill, to some extent, humans' social-emotional needs. Although this concept may be useful in understanding benefits that occur from pet ownership, it does not necessarily help explain benefits seen in brief AAT, as the time available may not be enough to allow formation of strong attachment. However, it is theorized that animals in this context may act as a transitional object, which provides comfort during the beginning of therapy until the client and therapist have formed a sufficient relationship (Katcher, 2000; Levinson, 1984; Triebenbacher, 1998). It is possible that this transitional type of relationship may be preferable in the context of AAT (Fine, 2006), as it may not be ethical or therapeutically helpful to encourage a deep attachment to a therapy animal with whom the client will not have continued contact.

Additionally, the Rogerian concepts of "nonevaluative empathy" and "unconditional positive regard" are frequently cited in human-animal interaction literature as things that animals can provide to humans (Katcher, 1983). It is often noted 
that animals seem to respond with various behaviors to people's emotions, reinforcing the idea that animals are able to sense what a person is feeling. Furthermore, these interactions have a nonjudgmental quality to them; animals appear to provide unconditional love, providing a confidante for potentially embarrassing or distressing conversations. A. M. Beck and Katcher (1996) note that over 30\% of an interviewed sample of pet owners stated that they confide in their pets. The confidential and nonjudgmental nature of the human-pet relationship may be particularly important to adolescents who feel socially isolated and vulnerable to criticism while revealing personal information (A. M. Beck \& Katcher, 1996). Taken a step further, animals appear to be not only nonjudgmentally accepting of humans but also of themselves. A psychologist who used cats frequently in psychotherapy stated:

Regarding the non-evaluation orientation, I use the cats to illustrate and make more real this Being aspect.... [A cat is] more focused on being than on being something. I have found that this example often goes a long way toward helping patients reorient their thinking in less negatively self-valuing ways. (Geis, 1969)

An animal's general orientation of empathy, nonjudgment, and acceptance may, therefore, facilitate therapy by providing an atmosphere emphasized as necessary by Carl Rogers, as well as provide a constant example of these traits throughout therapy. This specific quality of animals may be particularly complementary to mindfulness-based interventions that promote awareness and acceptance of one's current experience.

\section{Empirical Evidence for Animals in Psychotherapy}

Despite lacking a clear understanding of its mechanisms, there is some empirical support for the efficacy of animals in psychotherapy. Given the broad way in which 
animals have been used in healthcare settings, there are multiple terms used to refer to similar practices, including AAT, AAA, pet-facilitated therapy, pet therapy, pet-assisted therapy, AAT, and pet visitation (Connor \& Miller, 2000). The Delta Society used the terms "animal-assisted therapy" and "animal-assisted activities," distinguishing between the two practices (Delta Society, 1996). AAA is defined as providing an opportunity for various benefits to arise which enhance quality of life and can be delivered by a variety of trained helpers, including volunteers. On the other hand, AAT is defined as a "goaldirected intervention in which an animal meets specific criteria as an integral part of the treatment process." Furthermore, AAT must be delivered by a trained professional and the process should be documented and evaluated. Both AAT and AAA have been used with a variety of animals, in a variety of settings, and with a variety of populations and diagnoses.

A literature search revealed 14 empirical studies in peer reviewed journals pertaining to the effects of AAT/AAA on depressive symptoms. Six of these studies involved an elderly population. There is currently one meta-analysis that addresses the effectiveness of AAT/AAA on reducing depressive symptoms; however, there is no published meta-analysis on the effectiveness of reducing symptoms of anxiety. Nine empirical studies in peer reviewed journals were found pertaining to the effects of AAT/ AAA on anxious symptoms, all of which involved samples residing at hospitals or nursing homes, or receiving medical treatment. Furthermore, nine empirical studies in peer reviewed journals were identified pertaining to the effects of AAT/AAA on blood pressure and/or heart rate. Four of these studies involve samples from hospitals or nursing 
homes. For the purpose of the present literature review, five relevant studies on the use of AAT and/or animal-assisted activity with dogs (as defined by Delta Society) in addressing psychological distress (anxious and depressive symptoms) and physiological arousal (blood pressure and heart rate) will be reviewed. Studies that examined AAT/ AAA with elderly or nursing home residents were not chosen for further review, as results from elderly populations may be less relevant to a college population. The studies chosen for further review include one meta-analysis, two studies using a college sample, one study using a sample currently in treatment for a diagnosed mental disorder, and one study using a controlled experimental study design.

AAT and psychological distress. Souter and Miller (2007) conducted a metaanalysis to determine the effectiveness of AAT/AAA for alleviating depressive symptoms in humans. Studies had to use AAT or AAA, use a self-report measure of depression, have random assignment, include a control group, and report sufficient information to calculate effect sizes. After conducting an extensive literature search, Souter and Miller identified five studies that met the specified inclusion criteria (Brickel, 1984; McVarish, 1994; Panzer-Koplow, 2000; Struckus, 1989; Wall, 1994). Four out of the five included studies involved participants from nursing homes engaging in dog visitation; and the majority of the studies included predominantly female Caucasian participants. A mean difference effect size of 0.61 for the sample of studies was found to be statistically significant $(z=2.05, p<0.05)$, supporting the hypothesis that AAT/AAA is effective at decreasing depressive symptoms (positive difference scores reflect lower depressive symptoms in the AAA/AAT group.) The mean effect size is of medium magnitude, 
suggesting that while clients in AAT/AAA are unlikely to experience a dramatic decrease in depression, they will likely notice a lessening of symptoms (Cohen, 1988). Souter and Miller noted several methodological limitations in the existing literature, including a lack of randomized control comparisons, increased potential for experimenter bias, and poor generalizability. They also stated that there was a lack of research incorporating physiological measures related to health, and there is very little research addressing specific questions regarding the mechanisms underlying and best practices of AAT/AAA. In a study not included in the meta-analysis, Folse, Minder, Aycock, and Santana (1994) investigated the effects of AAT on depression in a college population. Participants meeting inclusion criteria based on scores on the Beck Depression Inventory (BDI) were assigned to group psychotherapy with AAT, animal-interaction in a group setting, or no treatment. Two dogs were used exclusively for each treatment group. A significant difference was found between posttest BDI scores of the animal-interaction group and control group, but not for the psychotherapy with AAT group. The authors theorized that this unexpected result may have occurred because of the emotionally difficult nature of psychotherapy experienced in the first group but not in the animal-interaction group. They also noted a difference in temperament between the two therapy dogs, suggesting that the more outgoing personality of the dog in the animal-interaction group may have influenced the between-group difference. Overall, the results of this study suggested that AAA may be part of an effective brief treatment for decreasing self-reported depressive symptoms in college students; however, further research with more rigorous methodology is needed to replicate these results. 
Barker and Dawson (1998) examined the effect of AAT on reducing anxiety in hospitalized psychiatric patients with a variety of diagnoses. Participants attended both an AAT group session and a therapeutic recreation group session; changes in state anxiety were compared for the same patients between these two conditions. Although no significant differences in anxiety change scores were found between the two treatment conditions, within-group differences were found for both treatments between disorder types. Only patients with mood disorders had a significant decrease in anxiety after participating in therapeutic recreation, while patients with mood, psychotic, and other disorders had a significant decrease in anxiety after participating in AAT. This result supports the idea that AAT is effective at decreasing anxiety in a broader range of patients than therapeutic recreation. However, the authors note the need for further research to determine if decreases in anxiety are maintained, as this treatment involved only a single session of therapy.

AAT and physiological arousal. Somervill, Kruglikova, Robertson, Hanson, and MacLin (2008) investigated the effects of physical interaction with dogs and cats on blood pressure and heart rate in college students. Students completed a screening questionnaire that included demographic information and attitudes toward pets. Then, in a series of five-minute intervals, students either talked casually with an experimenter or held a dog or cat in their laps. Blood pressure and heart rate were taken at the beginning and end of each interval. No differences in physiological arousal were found between subjects holding a cat compared to a dog. Statistical analyses revealed that diastolic blood pressure was slightly but significantly reduced after each animal-holding interval. 
However, significant reductions in both blood pressure and heart rate were found after comparing data from the three baseline intervals, indicating that a gradual reduction in physiological arousal occurred during the session. No differences in blood pressure were found between males and females while holding an animal; however, a significant increase in heart rate was recorded in females. Overall, the data mildly support previous findings that physical contact with an animal reduces blood pressure in humans; however, the authors note a need for further research to determine if this effect was related to holding an animal or simply exposure to the paradigm.

Cole, Gawlinski, Steers, and Kotlerman (2007) examined the effects of AAA on blood pressure, neurotransmitter levels, and state anxiety in patients hospitalized with heart failure. Patients experienced a brief animal visitation, a brief human visitation, or usual care. Data was collected before, during, and soon after treatment. The AAA group had significantly greater decreases in systolic artery pressure during and after treatment compared to the usual care group and significantly greater decreases in epinephrine and norepinephrine levels during and after treatment compared to the human visitation group. Furthermore, the AAA group had the greatest decrease in state anxiety compared to both the human visitation and usual care groups. These findings suggest that AAA improves cardiopulmonary pressure, neurohormone levels, and reduces anxiety in hospitalized patients with heart failure. Other studies have examined the effects of AAT/AAA on human stress response indicators, such as blood pressure, heart rate, hormone levels, and skin conductance, without measuring perceived anxiety; these studies have had mixed results. (For a review of further research, see Friedmann \& Tsai, 2006.) 
Summary of AAT findings. In summary, evidence from the literature provides some limited support for the use of AAA/AAT to decrease anxious and depressive symptoms as well as reduction of blood pressure and heart rate in certain populations. However, it is important to note that most of these studies were performed with hospitalized and/or elderly populations, allowing limited generalizability. Furthermore, these studies contained relatively small sample sizes and no attempts at replication have been made. Therefore, gaps in the available literature include a need for studies with rigorous methodology, the use of standardized definitions of AAA/AAT, studies that incorporate both self-report and physiological data, as well as exploration of underlying mechanisms, specifically the possibility that a therapy animal increases client participation and engagement.

\section{Mindfulness}

Mindfulness refers to the act of giving attention to one's current experience in an accepting and nonjudgmental way; and it was originally developed to achieve freedom from human suffering, as practiced in Buddhism. According to Kabat-Zinn (1990, p. 20), "The basic idea is to create an island of being in the sea of constant doing in which our lives are usually immersed, a time in which we allow all the 'doing' to stop." For Shapiro, Carlson, Astin, and Freedman (2006), mindfulness practice can be described as a process involving the interaction of three main elements: intention, attention, and attitude. These components occur simultaneously, informing each other, during the moment-tomoment cyclical process of practicing mindfulness.

Intention refers to why one is practicing and serves as a reminder of one's 
personal vision and values. Reflecting on intention allows one to determine what values are guiding mindfulness practice and if those values should be pursued or reevaluated. While intention is always present, it is also dynamic; one's reasons for practicing mindfulness can change over time. In fact, studies show that intentions tend to shift across a continuum from self-regulation, to self-exploration, and eventually to selfliberation as one continues to practice mindfulness (Mackenzie, Carlson, Munoz, \& Speca, 2007; Shapiro, 1992). Shapiro also found that many participants started practicing mindfulness with the goal of controlling specific symptoms associated with stress, pain, anxiety, or depression. Thus, intention may provide the motivation to engage in mindfulness practice.

Attention, the second aspect of mindfulness, describes the act of observing one's moment-to-moment external and internal experiences. Mindfulness practice involves refining one's ability to discern various inward responses, such as thoughts, emotions, and physical reactions, as well as deeply examine one's surroundings for sustained periods of time. Attention emphasizes the importance of staying involved with the present moment and is suggested to be critical to the healing process in psychotherapy (Shapiro \& Carlson, 2009).

Attitude, the third aspect of mindfulness, depicts the quality of engagement in practice. The attitudinal foundations of mindfulness include non-judgment, nonattachment, patience, and gentleness (Kabat-Zinn, 1990). Siegel (2007) developed the acronym COAL, which stands for curiosity, openness, acceptance, and love. It is these qualities that characterize the lens through which one attends to experiences while 
practicing mindfulness. Attitude is a way to relate to an experience, not change the event itself. Therefore, the attitude of kindness fosters the ability to give up control over internal and external experiences and provides an accepting framework from which one can attend to both enjoyable and painful events.

Mindfulness practice involves developing through first-hand experience the ability to attend to the current moment with an open attitude, which is often a new and challenging activity for people. There are two forms of practice: formal and informal (Kabat-Zinn, 1990). Formal practice consists of engaging in activities that specifically promote mindfulness and typically include various forms of mediation. Some examples of formal practice include mindful breathing, sitting mediation, and yoga. On the other hand, informal practice incorporates the act of being mindful during daily experiences, such as taking a shower or folding laundry. Through informal mindfulness practice, routine acts can become a new experience, while providing time for mindfulness to become more of a habit and less of a chore.

\section{A Brief Overview of Mindfulness in Western Psychology}

It is interesting to note that the concept of mindfulness originated from a form of meditation developed in the Buddhist tradition in Asia over 2,600 years ago. Yet in only the past 30 years has mindfulness been gradually woven into the practice of Western psychology, still maintaining its original intention as a tool for enhanced wellbeing and freedom from suffering. The term "mindfulness-based therapy" refers to any therapy in which the teaching of mindfulness practices is an explicit and key component of the 
treatment protocol (Shapiro \& Carlson, 2009). A decade ago, there were few empirical studies available to research the effects of mindfulness-based therapies. By 2008, Shapiro and Carlson found that there were 44 studies on mindfulness-based therapies funded by the National Institute of Health that year alone. It is therefore apparent that the interest in empirically examining the effects of mindfulness practice has increased greatly over the past 10 years.

The teaching of mindfulness practices is a component in four widely-known and currently used treatments; these treatments include MBSR (Kabat-Zinn, 1990), MBCT (Segal et al., 2002), Dialectical Behavior Therapy (DBT; Linehan, 1993), and Acceptance and Commitment Therapy (ACT; Hayes, Strosahl, \& Wilson, 1999). Other newer and less researched mindfulness-based therapies include mindfulness-based relapse prevention (Marlatt \& Gordon, 1985; Marlatt \& Witkiewitz, 2005) to treat clients with addiction problems, Mindfulness-Based Art Therapy (Monti et al., 2006) developed for use in medical populations, and Mindfulness-Based Eating Awareness Training (Kristeller, Baer, \& Quillian-Wolever, 2006) to treat individuals with binge eating disorder or obesity. These newer therapies, along with MBCT, are all based on the MBSR curriculum and have been modified to address the needs of various clinical populations. In contrast, DBT and ACT stem from other therapeutic backgrounds and incorporate additional primary components. Consequently, they will not be further examined in this review. Thus, for the purpose of this proposal, only literature involving MBSR, MBCT, and treatments based on these two therapies will be reviewed. 


\section{Empirical Evidence for Mindfulness}

MBSR was the first formalized mindfulness-based therapy (Kabat-Zinn, 1990). It was originally developed in 1979 as an alternative treatment for medical patients experiencing pain or long-term illness, but it was quickly generalized to various populations experiencing stress or anxiety. MBSR consists of an 8-week group program designed to provide intensive training in both formal and informal mindfulness practices. The program includes didactic learning, practice of techniques, facilitated group discussion, homework assignments, and a full-day mediation retreat. MBSR serves as a basic model in both medical and mental health fields for the teaching of mindfulness practices to promote healing and improved health.

Extending from the traditions of MBSR and cognitive therapy, MBCT was created to treat depression and prevent relapse (Segal et al., 2002). MBCT uses most of the same mindfulness practices found in MBSR and is typically provided in a similar 8session group format. Didactic teaching focuses on depression instead of stress. MBCT also includes some elements of cognitive therapy, such as examining the connections between affect and automatic thoughts. However, it maintains its theoretical foundation in mindfulness and does not promote changing or replacing negative thoughts. Both MBSR and MBCT are frequently used to treat clients with mood disorders, such as depression and anxiety.

A literature search in March 2013 revealed 136 empirical studies in peer reviewed journals pertaining to the effects of mindfulness on depressive symptoms and 117 studies on anxiety symptoms. The search also identified five meta-analyses addressing the 
effectiveness of mindfulness-based therapy (excluding DBT and ACT) in reducing depression and anxiety (Baer, 2003; Bohlmeijer et al., 2010; Grossman et al., 2004; Hofmann et al., 2010; Ledesma \& Kumano, 2008). In addition, 14 empirical studies in peer reviewed journals were identified pertaining to the effects of mindfulness on blood pressure and/or heart rate; eleven of which involved nonclinical samples. For the purpose of the present literature review, two meta-analyses (Baer, 2003; Hofmann et al., 2010) that explore the use of mindfulness-based therapy in addressing psychological distress (anxious and depressive symptoms) will be examined. These meta-analyses were chosen for further review because they include studies on populations with mental illnesses, making the results more relevant than the other three which focus on populations with physical illnesses. Meta-analyses by Grossman and colleagues, Ledesma and Kumano, and Bohlmeijer and colleagues suggested that mindfulness-based therapy is moderately effective at reducing psychological distress in patients with physical illnesses.) Two studies examining physiological arousal (blood pressure and heart rate) will be reviewed; they were chosen because they included methodology and populations more similar to the current study than what was included in the other studies.

Mindfulness and psychological distress. Baer (2003) conducted a meta-analysis to determine the effectiveness of mindfulness-based therapy for reducing symptoms of anxiety and depression in psychiatric and medical populations. Studies had to use a mindfulness-based intervention and have either a comparison group or pre- and postintervention data. No criteria for population were made. After conducting a literature search, 21 studies met the criteria. All of the included studies used MBSR, MBCT, or a 
program of similar duration based on MBSR; and samples included participants diagnosed with various DSM Axis I disorders, including depression and anxiety, various medical disorders, including chronic pain, as well as nonclinical populations, including college students. Results of the meta-analysis found that mindfulness-based therapy is moderately effective $(d=0.59)$ at reducing anxiety and depressive symptoms in clinical samples at posttreatment. Furthermore, the mean effect size at follow-up, which ranged from 3 months to 3 years, was found to be 0.59 as well, indicating moderate efficacy. An effect size for comparison treatments was not able to be calculated due to the small number of studies that included a control group and a lack of information about the nature of the comparison treatment. It should be noted that this review included a relatively small number of studies in total, approximately only half of which were of randomized control design. However, the author notes that these effect sizes are probably conservative, as many studies did not report exact data and the results of the metaanalysis deliberately erred on the side of assuming less effect. Gaps in the research as noted by the author include a need for randomized control design, larger sample sizes, described treatment integrity, and reports of clinical significance.

Hofmann and colleagues (2010) conducted a meta-analysis to determine the effectiveness of mindfulness-based therapy for reducing symptoms of anxiety and depression in psychiatric and medical populations. Studies had to use a mindfulnessbased intervention but not DBT or ACT, include an adult sample of participants with diagnosable medical or mental health disorders, include a pre- and posttreatment measure of anxiety and/or mood symptoms, and provide sufficient data to calculate effect sizes. 
After conducting an extensive literature search, 39 studies with a total of 1,140 participants met the specified inclusion criteria. All of the included studies used MBSR, MBCT, or a program of similar duration based on MBSR; and samples included participants diagnosed with anxiety disorders, depression, pain disorders, cancer, and other psychiatric and medical problems. In the overall sample, effect size estimates indicate that mindfulness-based therapy was moderately effective in improving anxiety (Hedge's $g=0.63$ ) and mood symptoms (Hedge's $g=0.59$ ) from pre- to posttreatment. Furthermore, in participants with depression and anxiety disorders, effect size estimates of $g=0.95$ for mood symptoms and $g=0.97$ for anxiety were found, supporting the idea that mindfulness-based therapy is largely effective for these populations. These results were unrelated to treatment type, duration, or publication year. Hofmann and colleagues suggest that their study provides further evidence for the efficacy of mindfulness-based therapy and its generalizability to various clinical populations. However, it is important to note that there was no required methodology for the studies reviewed, limiting the interpretation of these findings. Studies including randomization as well as a clearly defined control group are needed.

Mindfulness and physiological arousal. Kingston, Dooley, Bates, Lawlor, and Malone (2007) conducted a study to investigate the effects of mindfulness training on pain tolerance, physiological activity, and mood. The sample consisted of undergraduate students $(n=42)$ who partook to receive course credit. Participants were randomly assigned to a six-session mindfulness training or a two-session guided visual imagery training. Measures of pain tolerance (cold pressor test), mood, blood pressure, heart rate, 
and mindfulness skills were obtained before and after intervention. Results found that pain tolerance increased significantly for the mindfulness group but not the guided visual imagery group; pain intensity decreased, $F(1,40)=4.183, p=0.047$; and submersion time increased, $F(1,40)=4.183, p=0.047$. Diastolic blood pressure decreased from preto post-intervention in both groups; however, this finding was not statistically significant, $F(1,40)=3.892, p=0.055$. Furthermore, mean mindfulness scores on the Kentucky Inventory of Mindfulness Skills (KIMS) increased from pre- to posttreatment; however, a significant difference was not found between groups. It is unclear why measured mindfulness increased in the control group as well as the mindfulness-based intervention group. Results of this study indicate that mindfulness practice may increase pain tolerance as well as decrease blood pressure; however, the authors note that further research is needed for replication and to determine the potential mechanisms associated with this change.

Carlson, Speca, Faris, and Patel (2007) examined the effects of MBSR in cancer patients on psychological and physiological health. Participants all had a diagnosis of breast or prostate cancer. Exclusion criteria included current mood, anxiety, and psychotic disorder as well as previous experience with MBSR. Measures of stress, mood, blood pressure, and heart rate (among others) were taken before and after treatment, as well as at 6- and 12-month follow-up. Results found that self-reported stress scores decreased from pre- to posttreatment $(d=0.28)$ and were maintained at 12-month follow up with a moderate effect size $(d=0.40)$. However, no significant changes in mood were found. Systolic blood pressure decreased from pre- to post-intervention, $t(44)=2.02, p<$ 
0.05 , but diastolic blood pressure and heart rate had no significant change over time. The results suggest that MBSR may reduce anxiety symptoms as well as decrease blood pressure in patients with cancer. The authors note the need for further research to investigate the effects of MBSR on other physiological correlates of health.

Summary of mindfulness findings. In summary, evidence from the literature indicates that mindfulness-based therapy, particularly MBSR and MBCT, is moderately effective at reducing current anxiety and depressive symptoms in a variety of populations. Furthermore, there is some support for the efficacy of mindfulness-based therapy to reduce blood pressure in participants. However, it is important to note that many of these studies were performed with populations diagnosed with physical illness, allowing only cautious generalizability to either nonclinical populations or populations diagnosed with mental illness. Furthermore, the majority of available studies use a prepost intervention design rather than a randomized control group design, limiting the power of statistical analysis. Moreover, there is a lack of replication of studies examining the physiological effects of mindfulness practice, as well as a need for studies that incorporate both self-report and physiological data. The present study was designed to contribute to the empirically literature and address some of the limitations previous identified.

\section{Summary}

In review, evidence from the literature indicates that AAA/AAT may decrease anxious and depressive symptoms as well as reduces blood pressure and heart rate in 
certain populations. Further, mindfulness-based therapy, particularly MBSR and MBCT, is moderately effective at reducing anxiety and depressive symptoms in a variety of populations; and there is some support for the efficacy of mindfulness-based therapy to reduce blood pressure in participants. Given these findings, it is theorized that AAT may be particularly complementary to brief mindfulness training, in the form of a modified MBSR program, for people experiencing anxiety and/or depressive symptoms. It is hypothesized that the anti-anxiolytic features of a therapy animal may facilitate the practice of mindfulness in an individual therapy setting. Furthermore, it is hypothesized that the therapy animal will provide a focus for attention to one's current experience and exemplify acceptance and "being," enabling the understanding and practice of the main aspects of mindfulness. Gaps in the available literature addressed by the current study include a need for studies examining a non-medical population with rigorous methodology that incorporate both self-report and physiological data.

The following research questions were addressed in this study.

1. Does AAT in conjunction with mindfulness training facilitate the development of mindfulness skills compared to mindfulness training alone when assessed by self-report?

2. Does the presence of a therapy dog result in a greater reduction in anxiety during treatment, as assessed by self-report and physiological responses, compared to mindfulness training alone?

3. Does AAT in conjunction with mindfulness training result in a greater reduction in psychological distress, as evidenced by self-report, compared to mindfulness 
training alone?

4. Does the presence of a therapy dog increase satisfaction and engagement with treatment compared to mindfulness training alone? 


\section{CHAPTER III}

\section{METHODS}

This chapter includes the methods used in the study, including descriptions of participants, measures, and the intervention procedure.

\section{Participants}

Participants were recruited through Utah State University (USU) Student Services, including but not limited to counseling and psychological services, by referral, flyer, and/or email distributed with approval by the appropriate student service director. Participants were eligible to enroll in the study if they met the following inclusion criteria: (a) the individual must be experiencing at least mild psychological distress, as indicated by a total score above 53 on the OQ- 45 , and (b) the individual must have a selfreported positive attitude toward dogs. Participants were excluded if they (a) were currently receiving psychotherapy; (b) were planning to change a psychotropic medication in the near future; (c) were not capable of participating in light exercise due to physical/medical complications; (d) had been diagnosed with mental retardation or a developmental disability; (e) were allergic to dogs or reported not liking dogs; or (f) reported being skilled in mindfulness. Participants were screened using self-report questionnaires to provide demographic information, as well as assess attitude toward pets, current experience of psychological distress, and mindfulness. Subjects that were eligible, consented to participate, and completed the evaluations were compensated for their time by receiving a $\$ 20$ gift card to iTunes or Amazon at the end of the last session. 
A power analysis was conducted using $\mathrm{G}^{*}$ Power software (Faul, Erdfelder, Lang, \& Buchner, 2007), with an alpha level of .05 and power of .80. Meta-analyses for the efficacy of AAT on depressive symptoms (Souter \& Miller, 2007) and the efficacy of mindfulness-based therapy on anxiety and/or depressive symptoms (Hofman et al., 2010) both found medium effect sizes. A power analysis using a medium effect size of .25 specified a sample of 20 participants to detect a moderate effect in a repeated measures ANOVA across six points in time.

\section{Measures}

\section{Demographic Information}

Demographic data were collected through a survey measure created for the study. The measure included questions about sex, marital status, age, education, ethnicity/race, physical or medical conditions, current psychotherapy status, current psychotropic medication status, previous experience with mindfulness, and fear of dogs. The information was used to assess inclusion and exclusion criteria, as well as provide data for statistical control of demographic variables. Demographic information for the participants is included in Table 1.

Eighty-two people were screened, and 33 (40\%) were eligible for participation and were contacted. Of those, 26 (78\% of those contacted) started the intervention, and $21(81 \%)$ completed. Information on noncompleters is included in Table 2. The MBSR group contained 11 subjects (52\%), and the MBSR + AAT group contained $10(48 \%)$. The sample included 6 males (29\%) and 15 females (71\%) who had an average age of 25 
Table 1

Demographic Information

\begin{tabular}{lccc}
\hline Variable & MBSR $(n)$ & MBSR + AAT $(n)$ & Total $(n)$ \\
\hline Gender & 4 & 2 & 6 \\
Male & 7 & 8 & 15 \\
Female & & & \\
Ethnicity & 1 & 8 & 1 \\
African American & 8 & & 16 \\
Caucasian & 1 & 1 & 1 \\
Latino & & & 1 \\
Multiracial & 1 & 1 & 1 \\
Native American & & & 1 \\
Other & & & \\
\hline
\end{tabular}

Table 2

Information for Noncompleters

\begin{tabular}{|c|c|c|c|c|c|}
\hline Variable & $\begin{array}{c}\text { Noncompleter } \\
1 \\
\end{array}$ & $\begin{array}{c}\text { Noncompleter } \\
2\end{array}$ & $\begin{array}{c}\text { Noncompleter } \\
3\end{array}$ & $\begin{array}{c}\text { Noncompleter } \\
4\end{array}$ & $\begin{array}{c}\text { Noncompleter } \\
5\end{array}$ \\
\hline Group & MBSR & MBSR & MBSR & $\mathrm{MBSR}+\mathrm{AAT}$ & $\mathrm{MBSR}+\mathrm{AAT}$ \\
\hline Sex & Male & Female & Female & Female & Female \\
\hline Ethnicity & Caucasian & Caucasian & Caucasian & Caucasian & Caucasian \\
\hline $\begin{array}{l}\text { Pretreatment } \\
\text { OQ-45 }\end{array}$ & 88 & 59 & 95 & 74 & 54 \\
\hline $\begin{array}{l}\text { Sessions } \\
\text { completed }\end{array}$ & 1 & 1,2 & 1,2 & 1,2 & $1,2,3$ \\
\hline
\end{tabular}

$(S D=8.79)$, ranging from 18 to 54 years old. Seventy-five percent of the sample was Caucasian; the remainder of the sample included 1 African American (5\%), 1 Latino (5\%), 1 Native American (5\%), 1 Multiracial (5\%), and 1 self-identified Zambian 
participant $(5 \%)$. The average level of education was 2.5 years $(S D=1.66)$ of college. Eighty-six percent of the sample was single or separated, and the remaining $14 \%$ were married. The average score on the PAS-M was $102(S D=13.74$, with scores ranging from 77 to 122 , indicating that all subjects reported a positive attitude toward animals. The average score on the OQ-45 at screening was $83(S D=19.56)$, with scores ranging from 54 to $120 ; 76 \%$ of subjects had a total score above the clinical cutoff of 63 . Six participants $(29 \%)$ reported concurrent use of psychotropic medication.

\section{Pet Attitude Scale—Modified}

The Pet Attitude Scale-Modified (PAS-M; Munsell, Canfield, Templer, Tangan, \& Arikawa, 2004; Templer, Salter, Dickey, Baldwin, \& Veleber, 1981) is an 18-item questionnaire that measures attitudes toward pets. Questions are rated on a 7-point Likerttype scale, with ratings from 1 (strongly disagree) to 7 (strongly agree). Total scores can range from 18 to 126 . Higher scores reflect greater positive attitudes toward pets.

Although factor analyses have identified three factors: (a) love and interaction; (b) pets in the home; and (c) joy of pet ownership, the total score is generally used. The PAS total score has adequate internal consistency $(\alpha=.93)$ and 2-week test-retest reliability was $r$ $=.92$ (Templer et al., 1981). The PAS-M was developed to address concerns over three PAS items that assumed pet ownership. For these items, the phrase "or would if I had one" was added. The PAS-M total score also has adequate internal consistency $(\alpha=.92)$ and findings indicate no significant difference in responses between the PAS and the PAS-M (Munsell et al., 2004). Higher scores on the PAS were correlated with greater reduction in the mean arterial pressure and systolic pressure when petting dogs (Hama, 
Yogo, \& Matsuyama, 1996) and increased distress in AAT staff when the therapy dog disappeared (Crowley-Robinson \& Blackshaw, 1998). The PAS was found to correlate positively with childhood animal bonding (Brown, 2000). The PAS-M total score was used in the present study to assess inclusion criteria. The internal consistency reliability for the current sample was .90. (See Appendix B for measures used in this study.)

\section{Five Facet Mindfulness Questionnaire}

The Five Facet Mindfulness Questionnaire (FFMQ; Baer, Smith, Hopkins, Krietemeyer, \& Toney, 2006) is a 39-item self-report questionnaire that measures traitlike general mindfulness. The instrument is based on a factor analytic study of five independently developed and psychometrically sound mindfulness measures. Five factors emerged that appear to represent elements of mindfulness as it is currently conceptualized, including observing, describing, acting with awareness, nonjudging of inner experience, and non-reactivity to inner experience. Questions are rated on a 5-point Likert-type scale, with ratings from 1 (never or very rarely true) to 5 (very often or always true). Facet scores for observing, describing, acting with awareness, and nonjudging can range from 8 to 40 ; the range of scores for nonreactivity is 7 to 35 . Only facet scores are used for interpretation, with higher scores reflecting a greater tendency to be mindful in daily life. The facet scales all have adequate to good internal consistency with alpha coefficients ranging from .75 to .91. Individual facets of the FFMQ correlate positively with openness to experience, emotional intelligence, and self-compassion, and negatively with alexithymia, dissociation, and psychological distress (Baer et al., 2006). In the present study, facet scores were used to compare pre- and post-intervention trait- 
like mindfulness. The internal consistency reliabilities for the FFMQ facets for the current sample were .83 (observe), .93 (describe), .84 (act with awareness), .84 (nonjudge), and .88 (nonreact).

\section{Philadelphia Mindfulness Scale}

The Philadelphia Mindfulness Scale (PHLMS; Cardaciotto, Herbert, Forman, Moitra, \& Farrow, 2008) is a 20-item questionnaire that measures two trait-like components of mindfulness: present-moment awareness and acceptance. Questions are rated on a 5-point Likert-type scale, with ratings from 1 (never) to 5 (very often). Subscale scores can range from 10 to 50. Only subscale scores are used for interpretation, with higher scores reflecting higher levels of awareness and greater acceptance. Adequate internal consistency was demonstrated with both clinical and nonclinical populations, with alpha coefficients ranging from .75 to .87 . The subscales are not correlated with each other $(r=-.02, p>.05)$, indicating that awareness and acceptance can be examined separately. Significant differences were found between clinical and nonclinical populations, with nonclinical populations scoring higher (Cardaciotto et al., 2008). Further research is needed to determine test retest reliability. In the current study, subscale scores were used to compare pre- and post-intervention trait-like mindfulness. The internal consistency reliabilities for the awareness and acceptance scales in the current sample were .83 and .89 , respectively.

\section{Toronto Mindfulness Scale}

The Toronto Mindfulness Scale (TMS; Lau et al., 2006) is a 13-item self-report 
questionnaire created to assess state-like mindfulness, and is designed for use immediately following a meditation exercise. Evidence supports two factors: curiosity and decentering. Curiosity includes items that reflect awareness of the present moment with a quality of curiosity. Decentering includes items that emphasize awareness of one's experience with some psychological distance and disidentification. Items are rated on a 5point Likert-type scale, with ratings from 0 (not at all) to 4 (very much). Subscale scores can range from 0 to 24 for curiosity and 0 to 28 for decentering. Higher scores reflect greater mindfulness. Reliability estimates of the subscales, which are analogous to alpha coefficients for internal consistency, were found to be adequate at .86 (curiosity) and .87 (decentering). The criterion validity was supported by finding higher scores from participants after completing an 8-week MBSR program. The decentering subscale shows incremental validity in the prediction of perceived stress and distress. Subscale scores were used to assess state-like mindfulness after completing a mindfulness exercise at each session in the present study. The internal consistency reliabilities for the curiosity and decentering scales for the current sample were .79 and .78 , respectively.

\section{Beck Anxiety Inventory}

The Beck Anxiety Inventory (BAI; A. T. Beck \& Steer, 1990) is a 21-item selfreport questionnaire used to measure common symptoms of anxiety, such as nervousness and difficulty breathing. Items are rated on a 4-point Likert-type scale, with ratings from 0 (not at all) to 3 (severely). The total scores can range from 0 to 63 , with higher scores corresponding to higher levels of anxiety. Thirteen items measure physiological symptoms, five assess cognitive aspects, and three represent both cognitive and physical 
symptoms. Cutoff scores listed in the BAI manual are: 0-7 = minimal; 8-15= mild; 16-25 $=$ moderate and 26-63 = severe. The BAI has good psychometric properties $(\mathrm{A} . \mathrm{T} . \mathrm{Beck}$, Epstein, Brown, \& Steer, 1988; Fydrich, Dowdall, \& Chambless, 1992), demonstrating excellent internal consistency in a population of psychiatric outpatients with alpha coefficients ranging from .92 to .94 . The BAI has moderate concurrent validity with the Hamilton Anxiety Rating Scale (Hamilton, 1959) in 367 outpatients with anxiety disorders $(r=.56$; A. T. Beck \& Steer, 1991). In the current study, the BAI was used to compare pretreatment and posttreatment anxiety. The internal consistency reliability for the current sample was .79.

\section{State-Trait Anxiety Inventory, Form Y}

The State-Trait Anxiety Inventory, Form Y (STAI; Spielberger, 1983; Spielberger, Gorsuch, \& Lushene, 1970) is a 40-item self-report questionnaire that measures the subject's anxiety in the moment (A-State) and how anxious the subject generally feels (A-Trait). The A-State scale has been shown to be sensitive to change in transitory anxiety, and the A-Trait scale has been demonstrated to reflect a more stable quality. Questions are rated on a 4-point Likert-type scale, with ratings from 1 (not at all/almost never) to 4 (very much so/almost always). Scale scores can range from 20 to 80; higher scores reflect increased experienced anxiety and anxious symptoms. Internal consistency for the STAI is adequate for both scales, generally above .90 . Test-retest reliability ranges from .73 to .86 for the A-Trait. Test-retest reliability for the A-State is much lower as expected, ranging from .16 to .54 . Construct validity of both scales has 
been supported through evaluation of items and correlations with other anxiety scales (Smeets, Merckelbach, \& Griez, 1996). In the current study, the STAI was used to compare pretreatment and posttreatment trait anxiety (A-Trait) as well as assess change in state anxiety within and across intervention sessions (A-State). The internal consistency reliabilities for the two scales in the current sample were .89 (A-Trait) and .94 (A-State).

\section{Beck Depression Inventory-II}

The Beck Depression Inventory-II (BDI-II; Beck, Steer, \& Brown, 1996; Beck, Ward, Mendelson, Mock, \& Erbarugh, 1961) is a 21-item self-report questionnaire that is designed to assess somatic, affective, and cognitive symptoms of depression. Items are rated on a 4-point Likert-type scale, with ratings from 0 (reflecting an absence of the symptom) to 3 (reflecting a high level of the symptom). Total scores can range from 0 to 63, with higher scores reflecting greater symptoms of depression. Cutoff scores listed in the BDI-II manual are: 0-13 = minimal; 14-19= mild; 20-28= moderate; and 29-63= severe. The BDI-II has strong psychometric properties, with alpha coefficients greater than .89 and a 1-week test-retest reliability of .93 (Dozois, Dobson, \& Ahnberg, 1998). Beck and colleagues (1996) reported convergent validity with the Beck Hopelessness Scale $(r=.71)$ and the Hamilton Psychiatric Rating Scale for Depression $(r=.68)$. The authors also report divergent validity with the Beck Anxiety Inventory $(r=.60)$. The BDI-II was used to compare pre- and posttreatment depression in the present study. The internal consistency reliability for the current sample was .92 . 


\section{Outcome Questionnaire-45}

The Outcome Questionnaire-45 (OQ-45; Lambert \& Finch, 1999) is a 45-item self-report questionnaire designed to monitor clients' week-to-week progress in therapy. The OQ-45 has a total score and three subscales scores. The total score reflects overall level of disturbance and the subscales identify specific areas of difficulty for the patient. The subscales reflect subjective distress, quality of interpersonal relationships, and adequacy of social and occupational functioning. Items are rated on a 5-point Likert-type scale, with ratings from 0 (never) to 4 (almost always). Total scores can range from 0 to 180, with higher scores reflecting higher levels of distress. The clinical cutoff score listed in the OQ-45 manual is 63 . The OQ-45 has strong psychometric properties with oneweek test-retest reliability ranging from .78 to .84 . The OQ- 45 is also sensitive to changes that occur over short periods of time as a result of psychological intervention (Lambert et al., 2003). The OQ-45 total score was used in the present study to assess preand posttreatment distress and week-to-week progress during the intervention. The internal consistency reliability for the current sample was .89 .

\section{Session Engagement Questionnaire}

This is a four-item measure created for use in the present study. Items assessed participants' completion of homework during the previous week, as well as interest and engagement in the intervention. Items included: (1) How many times did you practice mindfulness in the past week? (2) How engaged were you in this session? (3) How much are you looking forward to attending the next session? (4) How mindful were you during this session? The last three questions were rated on a 10-point Likert-type scale, with 
ratings from 1 (not at all) to 10 (extremely). The measure was given at the end of every session and was used to track the level of client participation throughout the intervention.

\section{Client Satisfaction Questionnaire}

This is a 12-item measure created for use in the present study. The measure includes questions about the utility and acceptability of the intervention. Items included the following. Did you enjoy participating in this training? How effective was the therapist in facilitating the training? How likely are you to use the skills you learned in the future? Would you recommend a training like this to friends or family? Please rate your overall impression of your interactions with the therapist. Has participating in this training helped you to deal more effectively with your problems? In an overall, general sense, how satisfied are you with the training you have received? If you were to seek help in the future, would you participate in a similar intervention? Two items asked specifically about the inclusion of the therapy dog; these included the following. Would you have completed this training if there was no therapy dog? Did the therapy dog make this experience more helpful and/or enjoyable? Two items were open-ended questions that included the following. What aspects of the training did you find most helpful and/or enjoyable? What would you change about this experience? Items were rated on a 4-point Likert-type scale with ratings from 1 to 4 . Higher scores indicate higher satisfaction. The measure was given at the end of the last session and was used to assess treatment feasibility and compare client satisfaction between the control and dog groups. 


\section{Blood Pressure Monitor}

A digital automatic blood pressure monitor, the Omron BP785, was used to assess blood pressure and heart rate. This machine is self-inflating and has a universal cuff to help ensure proper fit and accurate readings. Pressure is accurate within $+/-3 \mathrm{mmHg}$ or $2 \%$, and heart rate is accurate within $+/-5 \%$ of the reading. This machine has been validated according to internationally accepted protocols (Coleman, Steel, Freeman, de Greeff, \& Shennan, 2008). Blood pressure and heart rate were assessed at three points throughout each session; data was used to assess change in physiological measures of anxiety within and across intervention sessions.

\section{Procedures}

All procedures were conducted with the approval of the USU Institutional Review Board. Participants were recruited through USU Student Services by referral, flyer, or approved email distributed by the service director. Interested participants contacted the student researcher and then received an email with the informed consent and a link to the online screening through Survey Monkey. The informed consent included a description of the purpose and procedures of the study, including an overview of the intervention process as well as eligibility criteria. After agreeing to participate in the study, participants were asked to complete the online screening questionnaires. This initial survey included Demographic Information, the Pet Attitude Scale - Modified, the Outcome Questionnaire-45, the Five Facet Mindfulness Questionnaire, the Philadelphia Mindfulness Questionnaire, the Beck Anxiety Inventory, and the Beck Depression 
Inventory-II (administered in that order) to assess for eligibility and provide baseline data. If eligibility criteria were met, participants were notified that they were eligible to participate in the intervention and then asked to schedule their first session with the student researcher. Participants could choose to receive phone calls or emails to remind them of their appointments. Reminder notices were sent the day before the scheduled appointment. Participants were randomly assigned to a modified MBSR program with or without AAT based on a numbers chart.

At each scheduled session, the participant was greeted in the lobby of the USU Psychology Community Clinic and escorted to a therapy room. The intervention procedure was followed as outlined in the intervention protocol, dependent on which session was being conducted (see Appendix A). During the beginning of the first and the end of the last intervention sessions, participants completed the following assessments: Five Facet Mindfulness Questionnaire, Philadelphia Mindfulness Questionnaire, Beck Anxiety Inventory, Beck Depression Inventory-II, and the Trait portion of the State/Trait Anxiety Inventory (A-Trait). At the beginning and end of every session, participants completed the State portion of State/Trait Anxiety Inventory (A-State). At the end of every session, participants completed the Toronto Mindfulness Scale, the Outcome Questionnaire-45, and the Session Engagement Questionnaire. At the end of the last session, participants also completed the Client Satisfaction Questionnaire. The participant was instructed by the experimenter to complete all self-report assessments on a tablet through Survey Monkey at the appropriate times during the beginning and end of each session. 
Blood pressure and heart rate were taken by an automatic blood pressure monitor while the client was seated three times throughout each session: at start, pre-mindfulness, and post-mindfulness. The cuff was secured on the upper arm by the experimenter. The automatic monitor assessed blood pressure and heart rate three times back-to-back and the average was immediately recorded by the experimenter electronically on an electronic tablet. The averaging procedure was recommended by the manufacturer of the monitor and confirmed as an accurate way to assess blood pressure and heart rate in independent trials (Coleman et al., 2008). Prior to the first measurement, the client was sitting for approximately 5 minutes or more to stabilize resting heart rate and blood pressure. All data were imported into an SPSS data file at the conclusion of the study. Participants who completed all 6 intervention sessions received a gift card worth $\$ 20$ at the end of the final session as compensation. Table A1 in Appendix A provides an overview of how time and materials were used in each session.

\section{Modified MBSR Intervention}

The intervention protocol consisted of a modified version of MBSR with or without AAT. MBSR was modified to use in an individual therapy format; the length of treatment was shortened from eight group therapy sessions to six individual therapy sessions, and the full-day retreat was excluded. Furthermore, increased emphasis was placed on exercises involving focus on the body and physical movement, with the goal of making the concepts of mindfulness more easily accessible to beginner learners and young adults. Both intervention conditions included six individual 50-minute sessions 
that occurred across 6 to 9 weeks, with the intention of having weekly sessions. Each session consisted of 15 minutes of didactic learning followed by 15 minutes of experiential learning. The other 20 minutes were used to collect physiological and selfreport data. The first and last sessions lasted 75 minutes to provide time to complete further data collection. The goals of the treatment protocol were to: (a) educate about mindfulness, (b) teach mindfulness techniques during session, (c) increase proficiency of mindfulness skills, and (d) promote the use of mindfulness to enhance health and wellbeing. Appendix A provides a summary of the didactic and experiential components (Table A2) and the intervention protocol.

\section{Considerations for the AAT Condition}

Participants in the AAT condition could interact with the dog as much or as little as they liked throughout each session; however, they were encouraged to be actively engaged with the dog at various times in both the didactic and experiential learning portions of the intervention. One dog was used in the study to control for differences in canine temperament as well as breed preference of participants. The dog was certified by the American Kennel Club as a Canine Good Citizen, as recommended by Fine (2006), and Delta Society as a therapy dog. The dog did not work more than two sessions in a row, or four sessions in a day, and had a brief walk and bathroom break every 2 hours. The dog had access to water at all times. Also, the dog was fully vaccinated and had a veterinary exam before the start of the study. Participants were allowed to interact with the dog for as long as the dog remained engaged. If the dog was judged to be nervous or otherwise uncomfortable, the dog was allowed to sit alone or leave, if deemed necessary 
by the therapist; however, this action was never taken and the dog remained in close proximity to the participants in all sessions. Participants who disliked or were phobic of dogs did not participate in the study, as per inclusion criteria. It was stated that if a participant requested the removal of the dog at any point, the dog would be removed and the participant could choose to end the session early. No participants requested the removal of the dog or to end a session early. Guidelines for the practice of AAT were adapted from recommendations by Fine (2006) for the safe and humane use of therapy animals in psychotherapy; these guidelines promote the safety of both the animal and the client.

\section{Intervention Piloting and Adherence}

The intervention protocol was reviewed by two licensed psychologists and written feedback was provided. Changes were made to the AAT-modified procedure to increase client interaction with the dog. The revised intervention was then piloted once with and once without AAT; and final revisions were completed based on client and therapist feedback. Time was extended for the first and last sessions to allow for completion of questionnaires. The author performed all sessions of the intervention under the supervision of a licensed psychologist. All of the intervention sessions were video recorded to monitor treatment integrity. Two tapes (10\%) were selected at random and evaluated by a licensed psychologist for consistency with the specified objectives, didactic content, experiential exercises, and AAT modifications indicated by the treatment manual, as well as general therapeutic skill. Treatment fidelity was determined to be satisfactory and consistency was found between reviewed sessions. 


\section{CHAPTER IV}

\section{RESULTS}

This chapter includes descriptive statistics for all variables, including pre- and posttreatment means for control (MBSR) and experimental (MBSR + AAT) groups. The rest of the chapter is organized by research questions posed in Chapter II. Each research question is addressed by providing the question, descriptions of analyses, and results.

\section{Descriptive Statistics}

Prior to addressing the research questions, descriptive statistics for all variables were calculated. Results of descriptive statistics as well as correlation tables for all measures are included in Appendix C. Skewness and kurtosis statistics, histograms, P-P plot, and box-and-whisker plots were examined for pretreatment variables for the total sample; based on an examination of the data, all measures can be assessed as having a normal distribution with no outliers based on skewness and kurtosis statistics and visual scrutiny of the graphed data. There were no significant differences in pretreatment means between the control and experimental groups (see Table 3).

Six data points were missing due to random recording error in the online survey program, including two sets of physiological data, two sets of subscale total scores from the Toronto Mindfulness Scale, and two total scores from the Outcome Questionnaire-45. Because missing data were minimal and did not alter individual scores on any measure, no method was used to replace them. Investigation of descriptive statistics indicates that the data can support substantive analyses. An alpha level of .05 was used for all analyses. 
Table 3

Comparison of Pretreatment Scores between Groups for the OQ-45, BDI-II, BAI, A-Trait, FFMQ, and PHLMS

\begin{tabular}{lcrcc}
\hline Variable & $d f$ & \multicolumn{1}{c}{$t$} & $\begin{array}{c}p \text { value } \\
(2 \text {-tailed })\end{array}$ & Cohen's $d$ \\
\hline OQ-45 & 19 & -.88 & .39 & -.40 \\
BDI-II & 19 & -1.23 & .23 & -.56 \\
BAI & 19 & .14 & .89 & .06 \\
A-Trait & 19 & -1.01 & .33 & -.46 \\
FFMQ & & & & \\
$\quad 19$ & .41 & .68 & .19 \\
$\quad$ Observing & 19 & -1.04 & .31 & -.48 \\
$\quad$ Describing & 19 & .05 & .96 & .02 \\
$\quad$ Act Aware & 19 & 1.95 & .07 & .89 \\
$\quad$ Nonjudging & 19 & .23 & .82 & .11 \\
$\quad$ Nonreacting & & & & \\
PHLMS & 19 & -.18 & .86 & -.08 \\
$\quad$ Awareness & 19 & .40 & .69 & .18 \\
$\quad$ Acceptance & & &
\end{tabular}

\section{Development of Mindfulness Skills}

The first research question asked if AAT in conjunction with mindfulness training facilitated the development of mindfulness skills compared to mindfulness training alone when assessed by self-report. This question was addressed with mixed and repeated measure ANOVAs.

General, or trait-like, mindfulness was assessed with the Philadelphia Mindfulness Scale (PHLMS) and the Five Facet Mindfulness Questionnaire (FFMQ) at the first and last sessions. Seven 2 (MBSR + AAT, MBSR) x 2 (pretreatment, 
posttreatment) factorial mixed ANOVAs were performed on the following variables: PHLMS: Awareness, PHLMS: Acceptance, FFMQ: Observe, FFMQ: Describe, FFMQ: Act Aware, FFMQ: Nonjudge, and FFMQ: Nonreact. The results are presented in Table 4. There was a statistically significant difference found between pretreatment and posttreatment scores on all measures of trait mindfulness; however, there was no statistically significant difference found between the MBSR and MBSR + AAT groups. A medium effect size, ranging from .53 to .69 , was found for all measures of trait mindfulness from pre- to posttreatment. The results indicate that both groups increased in their general mindfulness from the beginning to the end of the intervention.

State mindfulness was assessed with the Toronto Mindfulness Scale (TMS) immediately after the mindfulness exercise for each session. Two 2 (MBSR + AAT, MBSR) x 6 (sessions 1-6) factorial repeated measures ANOVAs were performed on the following variables: TMS: Curiosity and TMS: Decentering. The results are presented in Table 5. For the subscale of Curiosity, Mauchly's test of sphericity was significant, indicating that the assumption of sphericity had been violated, $\chi^{2}(14)=31.72, p=.01$; therefore, degrees of freedom were corrected using Greenhouse-Geisser estimates of sphericity $(\varepsilon=0.59)$. For the subscale of decentering, Mauchly's test of sphericity was nonsignificant, $\chi^{2}(14)=20.06, p=.13$, indicating that the variances of differences between conditions can be assumed to be equal. There was a statistically significant effect of treatment sessions on state mindfulness; however, there was no statistically significant difference found between MBSR and MBSR + AAT groups. Results indicate that when plotted, the data best fit a linear trend, demonstrating a medium to large effect 
Table 4

Comparison of Pre- and Posttreatment Trait Mindfulness

\begin{tabular}{|c|c|c|c|c|c|}
\hline Variables & & $d f$ & $F$ value & $p$ value & $E S\left(\eta_{\mathrm{p}}^{2}\right)$ \\
\hline \multicolumn{6}{|l|}{ PHLMS } \\
\hline \multirow[t]{3}{*}{ Awareness: } & Awareness & 1 & $36.09^{*}$ & .00 & .66 \\
\hline & Awareness*group & 1 & .00 & .99 & .00 \\
\hline & Error & 19 & & & \\
\hline \multirow[t]{3}{*}{ Acceptance: } & Acceptance & 1 & $21.11^{*}$ & .00 & .53 \\
\hline & Acceptance* group & 1 & .58 & .46 & .03 \\
\hline & Error & 19 & & & \\
\hline \multicolumn{6}{|l|}{ FFMQ } \\
\hline \multirow[t]{3}{*}{ Observe: } & Observe & 1 & $24.76^{*}$ & .00 & .57 \\
\hline & Observe*group & 1 & .00 & .98 & .00 \\
\hline & Error & 19 & & & \\
\hline \multirow[t]{3}{*}{ Describe: } & Describe & 1 & $22.18^{*}$ & .00 & .54 \\
\hline & Describe*group & 1 & .14 & .71 & .01 \\
\hline & Error & 19 & & & \\
\hline \multirow[t]{3}{*}{ Act aware: } & Act aware & 1 & $42.67^{*}$ & .00 & .69 \\
\hline & Act aware*group & 1 & .20 & .66 & .01 \\
\hline & Error & 19 & & & \\
\hline \multirow[t]{3}{*}{ Nonjudge: } & Nonjudge & 1 & $28.49^{*}$ & .00 & .60 \\
\hline & Nonjudge* group & 1 & .05 & .83 & .00 \\
\hline & Error & 19 & & & \\
\hline \multirow[t]{3}{*}{ Nonreact: } & Nonreact & 1 & $24.52 *$ & .00 & .56 \\
\hline & Nonreact*group & 1 & .41 & .53 & .02 \\
\hline & Error & 19 & & & \\
\hline
\end{tabular}

$* p<.05$.

size $(.71$ and .84$)$ of treatment time on state mindfulness. To investigate the significant findings, pairwise comparisons of mean differences in state mindfulness scores of the total sample were calculated between session 1 and each session thereafter. The results are presented in Table 6. Statistically significant differences in mean scores on both subscales of the TMS were found between sessions 1 and 5, and 1 and 6 indicating that participants' state mindfulness increased significantly by the fifth session but not before. 
Table 5

Comparison of State Mindfulness by Session

\begin{tabular}{llrrrr}
\hline Variables & & $d f$ & $F$ value & $p$ value & $E S\left(\eta_{\mathrm{p}}{ }^{2}\right)$ \\
\hline TMS & & & & & \\
Curiosity: & Curiosity & 2.97 & 14.29 & .00 & .47 \\
Greenhouse-Geisser: & Curiosity*group & 2.97 & .28 & .84 & .02 \\
& Error & 47.53 & & & \\
& Curiosity & 1 & $39.03^{*}$ & .00 & .71 \\
& Curiosity*group & 1 & .02 & .90 & .00 \\
& Error & 16 & & & \\
Decentering: & Decentering & 1 & $83.31^{*}$ & .00 & .84 \\
& Decentering*group & 1 & 1.26 & .28 & .07 \\
& Error & 16 & & & \\
\hline
\end{tabular}

$* p<.05$.

Table 6

Pairwise Comparisons of State Mindfulness Between Sessions

\begin{tabular}{llccc}
\hline Variables & & Mean difference & SE & $p$ value \\
\hline \multicolumn{1}{l}{ TMS } & & & & \\
\multicolumn{1}{c}{$\begin{array}{c}\text { Curiosity } \\
\text { Session 1 }\end{array}$} & Session 2 & -2.72 & 1.35 & .91 \\
& Session 3 & -3.06 & 1.46 & .80 \\
& Session 4 & -3.89 & 1.56 & .37 \\
& Session 5 & $-5.00^{*}$ & 1.19 & .01 \\
& Session 6 & $-9.94^{*}$ & 1.79 & .00 \\
Decentering & & & & \\
Session 1 & Session 2 & -3.06 & 1.25 & .40 \\
& Session 3 & -3.78 & 1.27 & .14 \\
& Session 4 & -4.22 & 1.37 & .11 \\
& Session 5 & $-6.17^{*}$ & 1.56 & .02 \\
& Session 6 & $-11.78^{*}$ & 1.26 & .00 \\
\hline
\end{tabular}

$* p<.05$. 


\section{Reduction in Anxiety During Treatment}

The second research question asked if the presence of a therapy dog resulted in a greater reduction in anxiety during treatment, as assessed by self-report and physiological responses, compared to mindfulness training alone. This question was addressed with repeated measure ANOVAs.

State anxiety was assessed with the State subscale of the State/Trait Anxiety Inventory (A-State) at the beginning and end of each session. One 2 (MBSR + AAT, MBSR) x 2 (presession, postsession) x 6 (sessions 1-6) factorial repeated measures ANOVA was performed on the variable of A-State. The results are presented in Table 7. Mauchly's test of sphericity was nonsignificant, $\chi^{2}(14)=16.29, p=.30$, indicating that the variances of differences between conditions can be assumed to be equal. There was a statistically significant effect of treatment time within and between sessions on state anxiety; however, there was no statistically significant difference found between the MBSR and MBSR + AAT groups. Results indicate that when plotted, the data best fit a linear trend, demonstrating a small to medium effect size of between-session treatment time (.42) and within-session treatment time (.57) on state anxiety. To investigate significant findings, a pairwise comparison of mean differences between pre- and postsession state anxiety scores from the total sample was calculated between session 1 and each session thereafter. The results are presented in Table 8. Statistically significant differences in mean change in scores on the State subscale of the STAI were found between sessions 1 and 2,3,4, and 6, indicating that participants became significantly less anxious at each session from pre- to post-session, except for the fifth session. It is 
Table 7

Comparison of Change in State Anxiety by Session

\begin{tabular}{lrccc}
\hline Variable & $d f$ & $F$ value & $p$ value & $E S\left(\eta_{\mathrm{p}}{ }^{2}\right)$ \\
\hline A-state session & 1 & $13.91^{*}$ & .00 & .42 \\
A-state session*group & 1 & 1.14 & .30 & .06 \\
Error & 19 & & & \\
& & & & \\
A-state pre/post & 1 & $24.71^{*}$ & .00 & .57 \\
A-state pre/post*group & 1 & .70 & .42 & .04 \\
Error & 19 & & & \\
& & & & .37 \\
A-state session*pre/post & 1 & $11.33^{*}$ & .00 & .02 \\
Session*pre/post*group & 1 & .30 & .59 & \\
Error & 19 & & & \\
$* p<.05$. & & &
\end{tabular}

Table 8

Pairwise Comparison of State Anxiety Change Between Sessions

\begin{tabular}{lllll}
\hline Variables & & $\begin{array}{c}\text { Mean } \\
\text { difference }\end{array}$ & SE & $p$ value \\
\hline A-state difference & & & & \\
Session 1: & Session 2 & $7.41^{*}$ & 2.19 & .05 \\
& Session 3 & $8.46^{*}$ & 2.25 & .02 \\
& Session 4 & $8.63^{*}$ & 2.26 & .02 \\
& Session 5 & 7.76 & 2.52 & .09 \\
& Session 6 & $9.03^{*}$ & 2.00 & .00 \\
\hline$* p<.05$ & & & &
\end{tabular}

possible that self-reported state anxiety did not decrease significantly during session 5 because the mindfulness exercise practiced during this session was sitting meditation. This exercise involves tracking thoughts, which can be difficult for clients and not as relaxing, as some thoughts they notice might be emotionally painful. 
Physiological anxiety was assessed by the measurement of blood pressure and heart rate at three times during each session. Three $2(\mathrm{MBSR}+\mathrm{AAT}, \mathrm{MBSR}) \times 3$ (baseline, pre-exercise, post-exercise) x 6 (sessions 1-6) factorial repeated measures ANOVAs were performed on the following variables: systolic blood pressure, diastolic blood pressure, and heart rate. The results of data on systolic blood pressure are presented in Table 9. Mauchly's test of sphericity was nonsignificant across sessions, $\chi^{2}(14)=$ $22.30, p=.08$, and within sessions, $\chi^{2}(2)=5.12, p=.08$, indicating that the variances of differences between conditions can be assumed to be equal. There was a significant effect of time within session on systolic blood pressure that fit a linear trend, supported with a small effect size of .33 .

The results of data on diastolic blood pressure are presented in Table 10.

Mauchly's test of sphericity was nonsignificant across sessions, $\chi^{2}(14)=16.14, p=.31$, and within sessions, $\chi^{2}(2)=1.98, p=.37$, indicating that the variances of differences

\section{Table 9}

\section{Comparison of Change in Systolic Blood Pressure by Session}

\begin{tabular}{lrccc}
\hline Variable & $d f$ & $F$ value & $p$ value & $E S\left(\eta_{\mathrm{p}}{ }^{2}\right)$ \\
\hline Systolic session & 1 & .49 & .50 & .03 \\
Systolic session*group & 1 & 1.03 & .33 & .06 \\
Error & 17 & & & \\
Systolic base/pre/post & 1 & $8.38^{*}$ & .01 & .33 \\
Systolic base/pre/post*group & 1 & .00 & .98 & .00 \\
Error & 17 & & & \\
Session*base/pre/post & 1 & 1.29 & .27 & .07 \\
Session*base/pre/post*group & 1 & 1.72 & .21 & .09 \\
Error & 17 & & & \\
$* p<.05$. & & & &
\end{tabular}


Table 10

Comparison of Change in Diastolic Blood Pressure by Session

\begin{tabular}{lrccc}
\hline Variable & $d f$ & $F$ value & $p$ value & $E S\left(\eta_{\mathrm{p}}{ }^{2}\right)$ \\
\hline Diastolic session & 1 & .55 & .47 & .03 \\
Diastolic session*group & 1 & 1.13 & .30 & .06 \\
Error & 17 & & & \\
& & & & .04 \\
Diastolic base/pre/post & 1 & .61 & .44 & .02 \\
Diastolic base/pre/post*group & 1 & .33 & .57 & \\
Error & 17 & & & .00 \\
Session*base/pre/post & 1 & .00 & .99 & .11 \\
Session*base/pre/post*group & 1 & 2.02 & .17 & \\
Error & 17 & & & \\
$* p<.05$. & & & &
\end{tabular}

between conditions can be assumed to be equal. No statistically significant effects were found for diastolic blood pressure.

The results of heart rate are presented in Table 11. Mauchly's test of sphericity was nonsignificant across sessions, $\chi^{2}(14)=9.09, p=.83$, and within sessions, $\chi^{2}(2)=$ $2.79, p=.25$, indicating that the variances of differences between conditions can be assumed to be equal. There was a statistically significant effect of within session time on heart rate that fit a linear trend, supported with an effect size of .45. Taken together, results of the physiological data analyses indicate that systolic blood pressure and heart rate decreased significantly within individual sessions.

\section{Reduction in Psychological Distress}

The third research question asked if AAT in conjunction with mindfulness training resulted in a greater reduction in psychological distress, as evidenced by self- 
Table 11

Comparison of Change in Heart Rate by Session

\begin{tabular}{lrccc}
\hline Variable & $d f$ & $F$ value & $p$ value & $E S\left(\eta_{\mathrm{p}}{ }^{2}\right)$ \\
\hline Heart rate session & 1 & 1.26 & .28 & .07 \\
Heart rate session*group & 1 & 1.04 & .32 & .06 \\
Error & 17 & & & \\
Heart rate base/pre/post & 1 & $13.79 *$ & .00 & .45 \\
Heart rate base/pre/post*group & 1 & 3.01 & .10 & .15 \\
Error & 17 & & & \\
Session*base/pre/post & 1 & .08 & .78 & .01 \\
Session*base/pre/post*group & 1 & .35 & .56 & .02 \\
Error & 17 & & & \\
$* p<.05$. & & & &
\end{tabular}

report, compared to mindfulness training alone. This question was addressed with mixed and repeated measure ANOVAs.

Psychological distress, including symptoms of depression and anxiety, as well as trait-like anxiety, was assessed with the Beck Depression Inventory-II (BDI-II), the Beck Anxiety Inventory (BAI), and the trait subscale of the State/Trait Anxiety Inventory (ATrait), respectively, at the first and last sessions. Three 2 (MBSR + AAT, MBSR) x 2 (pretreatment, posttreatment) factorial mixed ANOVAs were performed on the following variables: BDI-II, BAI, and A-Trait. The results are presented in Table 12. There was a statistically significant difference found between pretreatment and posttreatment scores on all measures of psychological distress; however, there was no statistically significant difference found between the MBSR and MBSR + AAT groups. A small to medium effect size, ranging from .32 to .68 , was found for all measures of psychological distress from pre- to posttreatment. The results indicate that both groups experienced decreased psychological distress from the beginning to the end of the intervention. 
Table 12

Comparison of Pre- and Posttreatment Distress

\begin{tabular}{llrccc}
\hline Variables & & $d f$ & $F$ value & $p$ value & $E S\left(\eta_{\mathrm{p}}{ }^{2}\right)$ \\
\hline BDI-II: & Session & 1 & $40.42^{*}$ & .00 & .68 \\
& Session*group & 1 & 3.65 & .07 & .16 \\
& Error & 19 & & & \\
BAI: & Session & 1 & $9.01^{*}$ & .01 & .32 \\
& Session*group & 1 & .00 & .98 & .00 \\
& Error & 19 & & & \\
STAI-Trait: & Session & 1 & $39.77^{*}$ & .00 & .68 \\
& Session*group & 1 & .98 & .33 & .05 \\
& Error & 19 & & & \\
\hline$* p<05$ & & & & &
\end{tabular}

$* p<.05$.

Psychological distress was also assessed with the Outcome Questionnaire-45 (OQ-45) at each session. One 2 (MBSR + AAT, MBSR) x 6 (sessions 1-6) factorial repeated measures ANOVA was performed on the variable of OQ-45. The results are presented in Table 13. Mauchly's test of sphericity was significant, indicating that the assumption of sphericity had been violated, $\chi^{2}(14)=43.71, p=.00$; therefore, degrees of freedom were corrected using Greenhouse-Geisser estimates of sphericity $(\varepsilon=0.45)$. There was a statistically significant effect of treatment time on OQ-45 scores; however, there was no statistically significant difference found between the MBSR and MBSR + AAT groups. Results indicate that when plotted, the data best fit a linear trend, demonstrating a medium effect size of treatment time (.54) on psychological distress as measured by the OQ-45. Findings support a significant decrease in self-reported psychological distress for both control and experimental groups from the beginning of the intervention to the end. To investigate the significant findings, pairwise comparisons of 
Table 13

Comparison of Psychological Distress by Session

\begin{tabular}{llcccc}
\hline \multicolumn{1}{c}{ Variable } & & $d f$ & $F$ value & $p$ value & $E S\left(\eta_{\mathrm{p}}{ }^{2}\right)$ \\
\hline OQ-45 & Session & 2.23 & $20.08^{*}$ & .00 & .54 \\
Greenhouse-Geisser & Session*group & 2.23 & .58 & .58 & .03 \\
& Error & 37.90 & & & \\
OQ-45 & Session & 1 & $39.34^{*}$ & .00 & .70 \\
& Session*group & 1 & .87 & .36 & .05 \\
& Error & 17 & & & \\
\hline
\end{tabular}

$* p<.05$.

mean differences in OQ-45 scores of the total sample were calculated between session 1 and each session thereafter. The results are presented in Table 14. Statistically significant differences in mean scores of the OQ-45 were found between session 1 and each session thereafter, indicating that participants' psychological distress decreased significantly after the first session.

\section{Client Satisfaction and Engagement}

The fourth question asked if the presence of a therapy dog increased satisfaction and engagement with treatment compared to mindfulness training alone. This question was addressed with repeated measure ANOVAs and paired $t$ tests.

Client engagement during session, including mindfulness, engagement, and positive anticipation for the next session, as well as how many times mindfulness was practiced during the previous week were assessed with the Session Engagement Questionnaire (SEQ). Two 2 (MBSR + AAT, MBSR) x 6 (sessions 1-6) factorial repeated measure ANOVAs were performed on the following one-item variables: Session 
Table 14

Pairwise Comparisons of Psychological Distress Between Sessions

\begin{tabular}{llccc}
\hline Variables & & Mean difference & $S E$ & $p$ value \\
\hline OQ-45 & & & & \\
Session 1 & Session 2 & $10.63^{*}$ & 1.87 & .00 \\
& Session 3 & $17.12^{*}$ & 2.85 & .00 \\
& Session 4 & $17.70^{*}$ & 2.61 & .00 \\
& Session 5 & $21.87^{*}$ & 3.26 & .00 \\
& Session 6 & $29.74^{*}$ & 4.63 & .00 \\
\hline
\end{tabular}

$* p<.05$.

Mindfulness and Session Engagement. The results are presented in Table 15. For both ANOVAs, Mauchly's test of sphericity was nonsignificant, $\chi^{2}(14)=12.78, p=.55$; $\chi^{2}(14)=19.22, p=.16$, indicating that the variances of differences between conditions can be assumed to be equal. There was a statistically significant effect of treatment time on scores of the items of Mindfulness and Engagement; however, there was no statistically significant difference found between the MBSR and MBSR + AAT groups. Findings suggest that the data best fit a linear trend; and medium to large effect sizes of .68 (mindfulness) and .47 (engagement) were found. To further investigate significant results, pairwise comparisons of mean differences for both scores for the total sample were calculated between session 1 and each session thereafter. The results are presented in Table 16. Statistically significant differences in mean scores on both items were found between sessions 1 and 5, and 1 and 6. Additionally, a significant difference in mean scores of mindfulness was found between sessions 1 and 4 . These results indicate that participants' self-reported mindfulness and engagement increased significantly by the fourth and fifth sessions, respectively. 
Table 15

Comparison of Change in Mindfulness and Engagement by Session

\begin{tabular}{lrccc}
\hline Variable & $d f$ & $F$ value & $p$ value & $E S\left(\eta_{\mathrm{p}}^{2}\right)$ \\
\hline Mindfulness session & 1 & $39.48^{*}$ & .00 & .08 \\
Mindfulness session*group & 1 & .00 & .96 & .00 \\
Error & 19 & & & \\
Engagement session & 1 & $16.98^{*}$ & .00 & .47 \\
Engagement session*group & 1 & .44 & .51 & .02 \\
Error & 19 & & & \\
$* p<.05$. & & & &
\end{tabular}

Table 16

Pairwise Comparison of Mindfulness and Engagement Between Sessions

\begin{tabular}{|c|c|c|c|c|}
\hline \multicolumn{2}{|l|}{ Variables } & Mean difference & $S E$ & $p$ value \\
\hline \multicolumn{5}{|c|}{ Mindfulness difference } \\
\hline \multirow{5}{*}{ Session 1} & Session 2 & -1.45 & .49 & .13 \\
\hline & Session 3 & -1.26 & .42 & .11 \\
\hline & Session 4 & $-1.91^{*}$ & .40 & .00 \\
\hline & Session 5 & $-2.65^{*}$ & .52 & .00 \\
\hline & Session 6 & $-2.46^{*}$ & .45 & .00 \\
\hline \multicolumn{5}{|c|}{ Engagement difference } \\
\hline \multirow[t]{5}{*}{ Session 1} & Session 2 & -.66 & .28 & .41 \\
\hline & Session 3 & -.57 & .28 & .82 \\
\hline & Session 4 & -1.12 & .38 & .13 \\
\hline & Session 5 & $-1.24^{*}$ & .34 & .03 \\
\hline & Session 6 & $-1.39 *$ & .33 & .01 \\
\hline
\end{tabular}

$* p<.05$.

Two 2 (MBSR + AAT, MBSR) x 5 (sessions) factorial repeated measure

ANOVAs were performed on the following one-item variables: Positive Anticipation and Practice. The results are presented in Table 17. For both ANOVAs, Mauchly's test of sphericity was nonsignificant, $\chi^{2}(9)=12.79, p=.17 ; \chi^{2}(9)=12.92, p=.17$, indicating that the variances of differences between conditions can be assumed to be equal. There were no significant effects for positive anticipation or practice. 
Table 17

Comparison of Change in Positive Anticipation and Practice by Session

\begin{tabular}{lrccc}
\hline Variables & $d f$ & $F$ value & $p$ value & $E S\left(\eta_{\mathrm{p}}{ }^{2}\right)$ \\
\hline Anticipation session & 1 & .83 & .38 & .04 \\
Anticipation session*group & 1 & .00 & 1.00 & .00 \\
Error & 18 & & & \\
& & & & \\
Practice session & 1 & .07 & .80 & .00 \\
Practice session*group & 1 & 1.29 & .27 & .06 \\
Error & 19 & & & \\
$* p<.05$. & & &
\end{tabular}

Client satisfaction was assessed with the Client Satisfaction Questionnaire (CS). Seven independent samples $t$ tests were performed, one for each item of the survey, except the first item regarding enjoyment of the training which had no variance in response (mean $=4.0, S D=0)$ and could not be examined further (see descriptive statistics). The results are presented in Table 18. Levene's test for homogeneity of variances was significant for the items regarding efficacy of therapist, recommending the training, and likelihood of future participation; therefore a $t$ test not assuming homogeneous variances was calculated for those items. No statistically significant differences in scores on the CS items were found between the MBSR and MBSR + AAT groups; however, given the large negative effect sizes found, follow up studies with increased power might produce results that show significant differences between groups, specifically higher ratings by the MBSR + AAT group of therapist efficacy, recommending the training, and participating in future treatment. Additionally, the results of the two questions asked only of the MBSR + AAT group indicate that, although subjects agreed they would have participated without the dog, they strongly endorsed that 
Table 18

Comparison of Client Satisfaction Between Groups

\begin{tabular}{|c|c|c|c|c|c|c|}
\hline \multirow[b]{2}{*}{ Variables } & \multicolumn{2}{|c|}{ Levene's test } & \multicolumn{4}{|c|}{$t$ test } \\
\hline & $F$ value & $\begin{array}{c}p \\
\text { value }\end{array}$ & $t$ & $d f$ & $\begin{array}{c}p \text { value } \\
\text { (2-tailed) }\end{array}$ & Cohen's $d$ \\
\hline \multicolumn{7}{|l|}{ Effective therapist } \\
\hline Equal variances assumed & 13.29 & .00 & -1.42 & 19 & .17 & -.65 \\
\hline Equal variances not assumed & & & -1.49 & 10.00 & .17 & -.94 \\
\hline \multicolumn{7}{|l|}{ Use the skills } \\
\hline Equal variances assumed & 4.55 & .05 & -.98 & 19 & .34 & -.45 \\
\hline \multicolumn{7}{|l|}{ Recommend the training } \\
\hline Equal variances assumed & 10.40 & .00 & -1.42 & 19 & .17 & -.65 \\
\hline Equal variances not assumed & & & -1.45 & 16.99 & .17 & -.70 \\
\hline \multicolumn{7}{|l|}{ Interaction with therapist } \\
\hline Equal variances assumed & .02 & .89 & .07 & 19 & .95 & .03 \\
\hline \multicolumn{7}{|l|}{ Deal more effectively } \\
\hline Equal variances assumed & 3.29 & .09 & -.50 & 19 & .63 & -.23 \\
\hline \multicolumn{7}{|l|}{ Satisfied with training } \\
\hline Equal variances assumed & 1.26 & .28 & .59 & 19 & .56 & .27 \\
\hline \multicolumn{7}{|l|}{ Participate in future } \\
\hline Equal variances assumed & 5.14 & .04 & -1.66 & 19 & .11 & -.76 \\
\hline Equal variances not assumed & & & -1.67 & 18.76 & .11 & -.77 \\
\hline
\end{tabular}

the dog made the intervention more helpful and enjoyable (see Appendix C: Table C6 for descriptive statistics).

Examination of the open-ended questions indicates that clients were generally satisfied with their experience and had few suggestions for improving the intervention. Participants noted aspects of mindfulness, specific experiential exercises, the treatment process, positive qualities of the therapist, and the presence of the dog as most helpful and/or enjoyable. Suggestions for change included wanting to have been in the AAT 
group and increasing the amount of sessions. Fourteen of the 21 participants either did not respond to the question or said they would change nothing. 


\section{CHAPTER V CONCLUSIONS}

This chapter offers a review of the purpose of the study and a brief summary of the results. Next, the results of the current study are interpreted in relation to the previous literature on AAT and MBSR, as discussed in the literature review found in Chapter II. Additionally, limitations of the current study are examined and suggestions for future research are provided. Lastly, recommendations for clinical practice are offered.

\section{Purpose of the Study}

The current study attempted to examine the potential psychological and physiological effects AAT might add to a modified MBSR program. In addition to determining if AAT as an adjunctive therapy affects treatment results, the current study also aimed to address several noted gaps in the existing literature, including a lack of rigorous methodology, a lack of multiple sources of data, and a lack of a generalizable sample. The current study addressed these gaps by using a randomized control trial design, the measurement of both physiological and self-reported psychological change, and a college outpatient sample that sought treatment for a variety of clinical issues.

\section{Summary of Results}

In general, results of the study support the efficacy of the modified MBSR program; however, the interaction with a therapy dog had no significant psychological or physiological effects on the participants. All participants reported an increase in both 
state and general, or trait-like, mindfulness. Specifically, state mindfulness increased significantly by the fifth session. State anxiety was reduced for all participants both within each session and across all six sessions. Furthermore, systolic blood pressure and heart rate decreased significantly within sessions for all participants. Psychological distress, including anxiety and depressive symptoms, significantly decreased for all participants over the six-session intervention. Additionally, all participants rated engagement and satisfaction with the intervention as very high.

Results indicate that the intervention was efficacious at teaching the practice of mindfulness; participants learned how to place their attention in the present moment nonjudgmentally. Participants experienced a psychological and physiological decrease in anxiety during the sessions. They reported feeling more relaxed after practicing mindfulness. The intervention also effectively reduced psychological distress; participants reported experiencing fewer anxiety and depressive symptoms. Findings of linear trends between and within sessions indicate that participants experienced continual change during the intervention. Furthermore, the training was feasible and acceptable to the sample studied. Based on the data collected, the addition of AAT did not significantly enhance or hinder the desired treatment outcomes; however, results of effect sizes suggest that AAT increased some aspects of client satisfaction, particularly perception of therapist efficacy, recommendation of the intervention, and future participation in similar interventions.

Besides direct findings, it is important to consider some additional aspects of the treatment protocol when considering the results of the current study. Because this study 
was based on the premise that MBSR is an effective intervention, AAT was being added to a treatment that already demonstrated efficacy. Therefore, it became difficult to distinguish differences between treatment conditions based on the dependent variables examined. When considering the modified MBSR program for future use, results indicate that the number of intervention sessions provided was adequate to produce a significant change in the development of mindfulness skills and the reduction of psychological distress. Additionally, the data also suggest that less than five sessions may not be enough for the acquisition of mindfulness skills. All participants rated satisfaction with the intervention as very high, indicating that the modified mindfulness training, with or without AAT, is likely a feasible intervention in a moderately distressed college population.

In addition to examining the treatment protocol, it is important to note some aspects of the interactions between therapist, participant, and dog as part of the study results. Often times, the dog was talked about at the beginning of session. Participants asked questions about the dog, including his sex, age, breed, and favorite activities. The therapist reported being aware that she intentionally used the dog as an icebreaker to provide a topic of discussion. Furthermore, the student therapist reported that she enjoyed using the dog as a way to build rapport. Also, participants responded to the dog's behaviors and appearance, sometimes laughing at the dog's profuse tail wagging or commenting on his "smile." Throughout the training, the student therapist observed varying amounts and differing qualities of physical contact between the dog and participant. Many participants encouraged the dog to lie next to them on the couch, 
petting the dog throughout the session. One participant rarely touched the dog, noting that she did not want fur on her clothes. In general, interaction between the participant and the dog varied between participants; however, all participants spontaneously interacted verbally or physically with the dog.

\section{Addressing Gaps in the Literature}

The extant literature on AAT and MBSR has a number of significant limitations that were addressed in the present study, including poor methodology, few sources of data, vague terminology, and limited generalizability of findings. The following sections will discuss each of these concerns and integrate the results of the current study.

\section{Methodological Rigor}

The research surrounding human-animal interactions generally lacks rigorous methodology. In particular, many studies fail to include a control comparison group. The present study utilizes a randomized control design to tease apart the effects of the therapy

dog. Therefore, a more rigorous methodology allowed for a more in-depth investigation of AAT. Additionally, researchers frequently neglected to include adequate information regarding effect sizes, making comparisons between studies difficult. The current study provides this necessary information.

Existing literature in the field of mindfulness-based interventions suffers some of the same limitations as current research on AAT. In particular, there is a need for more research with clearly defined treatment protocols. The current study utilized an MBSR program that was modified to fit an individual therapy format and a shortened session 
sequence. Results support the efficacy of the modified design in both increasing mindfulness skills and decreasing psychological distress. Moreover, the individual therapy format might be more appealing to college students who resist the idea of group therapy but could benefit from mindfulness training.

\section{Multiple Data Sources}

The majority of previous research relied on client self-report and therapist observer-report for assessment of the effects of the therapy animal. As noted in other critiques of the available literature, there is a potential bias in self-report and the possibility to overestimate the impact of AAT. The current study attempts to reduce or eliminate observer bias by using both psychological and physiological data to assess anxiety throughout the intervention. Results of analyses of blood pressure and heart rate data supported the findings of participants' self-report data suggesting that, at least in this case, self-report data did not have a positive bias. Also, the additional collection of physiological data might help determine underlying mechanisms associated with previous reports of physical and mental health benefits by providing insight into how the human body responds to interaction with an animal.

Existing literature on mindfulness lacks physiological data in conjunction with psychological data. Similar to the field of AAT, studies often provide either physiological or psychological assessment but not both. Results of the current study support previous findings that mindfulness practice can reduce systolic blood pressure and heart rate. Additionally, these findings are supported by client self-report of experiencing fewer anxious symptoms. Furthermore, psychological distress was assessed by multiple 
measures, including participant self-report of anxiety and depressive symptoms, as well as trait-like anxiety and week-to-week distress. The examination of physiology to support client report of psychological constructs might lead to a better understanding of the mechanisms of mindfulness practice associated with positive mental health change.

\section{Specific Terminology}

In the field of human-animal interaction, some previous studies failed to differentiate between "animal-assisted activities" and "animal-assisted therapy," or researchers and clinicians have used the terms interchangeably or incorrectly. As stated in the literature review, both terms have distinct definitions, with AAT indicating an intentional use of the therapy animal in meeting treatment goals. Therefore, it is necessary to employ the correct term when providing research results because the terms denote different levels of planning, different goals, and, often, different lengths of exposure to the therapy animal. The current study employs the term "AAT" because interaction with the therapy dog was used to address specified goals of the intervention, namely the practice of mindfulness.

\section{Generalizability}

Another gap in our understanding of the effects of therapy animals on human health is to whom is AAT is applicable. The bulk of previous research on AAT has involved elderly populations and/or participants with undefined diagnoses. As AAT is increasingly used in a variety of populations, particularly college settings, it is necessary to understand the impact in younger samples with broadly but clearly defined mental 
health problems. The current study provides results that are generalizable to clients seeking services at university counseling centers. It is interesting to note that the majority of the current sample was recruited from the counseling and psychological services center, indicating that this intervention was acceptable and feasible to the typical student seeking mental health services.

Some of the current research on mindfulness-based interventions examines samples with physical illnesses, such as chronic pain (see meta-analysis by Hofmann et al., 2010). This literature does not allow for generalization to other populations, particularly samples with mental health problems. The current study provides evidence for the beneficial effects of mindfulness training in a college sample experiencing psychological distress. Results support the use of structured mindfulness training in university counseling centers to aid in the reduction of anxious and depressive symptoms. Because of the structured nature of the intervention protocol, it may be possible to train peer mentors or trainees to provide the training to distressed students, reducing the work load of licensed therapists while still providing effective services. Additionally, it should be noted that the current protocol involved only 30 minutes per session of direct intervention, indicating that significant improvement in certain domains of psychological health was seen in an abbreviated period of time compared to a traditional 50-minute individual therapy session.

\section{Limitations and Future Research}

This study had a variety of limitations that could be addressed in future research. 
First, issues surrounding methodology are discussed. The small sample size of the current study limits interpretation of effect sizes; therefore, future research must include larger sample sizes to increase power. Additionally, the current sample consists predominantly of Caucasian participants, limiting the generalizability of the findings to other ethnicities. Many universities have large populations of ethnic minority students, both domestic and international; therefore, it is necessary to expand the current study to include a more diverse ethnic sample. Furthermore, it should be noted that the averaging procedure used to assess blood pressure and heart rate could potentially cause elevated results due to the process of repeatedly inflating the cuff. Although the procedure used in the current study was recommended by two sources, future studies could be improved by comparing various procedures to determine any potential effects of repeatedly inflating the cuff.

The current study used one therapist and one therapy dog; although this design provided control for differences in therapist style and temperament of the dog, it does not allow for the results to be generalized across therapist/dog teams. Previous research has shown that dog temperament influences results, including the amount of interaction the client has with the dog as well as the social perception attributed to the therapist. Thus, future research must further explore the impact of therapist style and dog temperament by including multiple therapist/dog teams.

In the current study, client satisfaction with the intervention was high. However, interpretation of these results is limited because the questionnaire used was created specifically for this intervention and, therefore, not assessed to be psychometrically sound. Future research should include an established measure to assess client satisfaction. 
Potentially because of the ceiling effect, it was impossible to determine if a difference in satisfaction was present between the control and experimental groups. It is interesting to note that the study was advertised by promoting the incorporation of the therapy dog, as it was referenced in the imagery and language on the fliers and during verbal referrals by student service staff. It would be interesting to further assess the potential influence AAT might have on recruitment by comparing participant interest when exposed to standard advertisement and animal-influenced advertisement. In the current study, two participants in the control group asked if they could switch to the AAT group after discovering their random assignment in the first session, noting they wanted to interact with the therapy dog. Therefore, the therapy animal might act as a selling point for mental health services. Future research is needed to explore the type of client AAT might attract, including demographic information, personality characteristics, and symptom clusters.

The current study utilized a modified MBSR intervention; however, the acceptability of the control situation in the college population studied was previously unknown. Results support the feasibility of the intervention; yet it is unknown how the addition of AAT would impact the feasibility of a generally less acceptable intervention in the population. A future study could examine the impact of complementing a low-level noxious intervention with AAT to better explore how AAT affects treatment acceptability. Because of the stigma associated with mental health services, it would be interesting to examine the potential destigmatizing role of the therapy animal, as discussed in existing literature.

Although results of the current study do not support an advantage of adding AAT 
to mindfulness training, it is possible that critical variables were not assessed during the intervention. For example, level of client interaction with the dog was not measured. Specific mindfulness exercises encouraged direct contact with the therapy dog; however, physical contact between the participant and the dog in the experimental group varied greatly. Some participants invited the dog to lie next to them or on their laps on the couch, while others directed the dog to lie on the floor. Future research would benefit from observational data to explore the quantity and quality of client-dog interaction during sessions. Additionally, client-therapist rapport was not assessed in the current study. Given results of previous research indicating the influence of a companion or service animal on perception of owner/handler personality, it would be interesting to measure the client's perception of the therapist and the therapeutic bond.

In conclusion, this study supports the mental health benefits of the modified MBSR program used; however, AAT was not demonstrated to significantly enhance the intervention, as measured by the assessments used in the present study. Given the large effect sizes found, it is possible that AAT may have increased client satisfaction compared to MBSR alone. Future research should address how AAT might influence client interest in treatment and therapeutic alliance. Due to the currently somewhat indefinable nature of human-animal interaction, it is possible that the use of a qualitative or mixed research design would more effectively address the questions left unanswered.

\section{Recommendations for Clinical Practice}

Based on the existing literature and the findings of the current study, a brief list of 
recommendations for clinical practice involving MBSR and AAT are offered.

1. Mindfulness training is suggested as an effective intervention for college students experiencing a variety of clinical issues. A minimum of five sessions is necessary to achieve an increase in skills; however, sessions can be 30 minutes long. Because clients may not be "engaged" until the fourth session, building a system to help clients attend sessions during the learning phase may be particularly important.

2. It is recommended that multiple experiential exercises be used when teaching mindfulness skills, as clients indicated that they preferred different exercises and enjoyed the variety in experiences.

3. Therapy animals may offer incentive to some people to initiate and/or engage with mental health services. It is recommended that AAT be considered as a way to reduce stigma, draw clients, and attractively package treatment. This use might be particularly effective in a college setting where many students are not permitted to have companion animals.

4. It is recommended that clinicians be thoughtful of how the presence of a therapy animal affects their own attention and mood. An animal can be distracting or relaxing, influencing the therapist's interactions with the client.

5. Clinicians are encouraged to intentionally explore ways in which a therapy animal can increase therapeutic alliance. For example, animals can provide touch; offering physical contact through the therapy animal is generally a safer alternative, allowing the client to control the interaction more effectively. 


\section{REFERENCES}

Allderidge, P. H. (1991). A cat, surpassing in beauty, and other therapeutic animals. Psychiatric Bulletin, 15, 759-762.

American Psychological Association (APA). (2010). Society of clinical psychology, American Psychological Association, Division 12: Psychological treatments. Retrieved from http://www.div12.org/PsychologicalTreatments/treatments.html

American Veterinary Medical Association (AVMA). (2007). US pet ownership and demographics sourcebook. Schaumburg, IL: Author.

Baer, R. (2003). Mindfulness training as a clinical intervention: A conceptual and empirical review. Clinical Psychology: Science \& Practice, 10(2), 125-143. doi:10.1093/clipsy/bpg015

Baer, R. A., Smith, G. T., Hopkins, T., Krietemeyer, J., \& Toney, L. (2006). Using selfreport assessment methods to explore facets of mindfulness. Assessment, 13, 2745.

Barker, S. B., \& Dawson, K. S. (1998). The effects of animal-assisted therapy on anxiety ratings of hospitalized psychiatric patients. Psychiatric Services, 49, 797-801.

Beck, A. M., \& Katcher, A. (1996). Between pets and people: The importance of animal companionship. West Lafayette, IN: Purdue University Press.

Beck, A. M., \& Katcher, A. (2003). Future directions in human-animal bond research. American Behavioral Scientist, 47(1), 79-93.

Beck, A.T., Epstein, N., Brown, G., \& Steer, R.A. (1988). An inventory for measuring clinical anxiety: Psychometric properties. Journal of Consulting \& Clinical Psychology, 56, 893-897.

Beck, A.T., \& Steer, R.A. (1990). Manual for the Beck Anxiety Inventory. San Antonio, TX: Psychological Corporation.

Beck, A.T., \& Steer, R.A. (1991). Relationship between the Beck Anxiety Inventory and the Hamilton Anxiety Rating Scale with anxious outpatients. Journal of Anxiety Disorders, 5, 213-223.

Beck, A. T., Steer, R. A., \& Brown, O.K. (1996). Beck Depression Inventory manual (2 ${ }^{\text {nd }}$ ed.). San Antonio. TX: Psychological Corporation.

Beck, A. X, Ward, C. H., Mendelson, M., Mock, J., \& Erbaugh, J. (1961). An inventory for measuring depression. Archives of General Psychiatry, 4, 561-571. 
Bohlmeijer, E., Prenger, R., Taal, E., \& Cuijpers, P. (2010). The effects of mindfulnessbased stress reduction therapy on mental health of adults with a chronic medical disease: A meta-analysis. Journal of Psychosomatic Research, 68, 539-544. doi:10.1016/j.jpsychores.2009.10.005

Bossard, J. H. S. (1944). The mental hygiene of owning a dog. Mental Hygiene, 28, 408413.

Brown, J. M. (2000). Childhood attachment to a companion animal and social development of incarcerated male juvenile delinquents. Dissertation Abstracts International, 60, 5809.

Brickel, C. M. (1984). Depression in the nursing home: A pilot study using pet-facilitated psychotherapy. In R. K. Anderson, B. L. Hart, \& L. A. Hart (Eds.), The pet connection: Its influence on our health and quality of life (pp. 407-415). Minneapolis, MN: Center to Study Human-Animal Relationships \& Environments, University of Minnesota.

Brickel, C. M. (1985). Initiation and maintenance of the human-animal bond: Familial roles from a learning perspective. Marriage Family Review, 8(3/4), 31-48.

Cardaciotto, L., Herbert, J. D., Forman, E. M., Moitra, E., \& Farrow, V. (2008). The assessment of present-moment awareness and acceptance: The Philadelphia mindfulness scale. Assessment, 15, 204-223.

Carlson, L. E., Speca M., Faris, P., \& Patel, K. (2007). One year pre-post intervention follow-up of psychological, immune, endocrine and blood pressure outcomes of mindfulness-based stress reduction (MBSR) in breast and prostate cancer patients. Brain, Behavior, \& Immunity, 21, 1038-1049.

Chandler, C. (2005). Animal assisted therapy in counseling. New York, NY: Routledge.

Cohen, J. (1988). Statistical power analysis for the behavioral sciences ( $2^{\text {nd }}$ ed.). Hillsdale, NJ: Erlbaum.

Cole, K. M., Gawlinski, A., Steers, N., \& Kotlerman, J. (2007). Animal-assisted therapy in patients hospitalized with heart failure. American Journal of Critical Care, 16, 575-585.

Coleman, A., Steel, S., Freeman, P., de Greeff, A., \& Shennan, A. (2008). Validation of the Omron M7 (HEM-780-E) oscillometric blood pressure monitoring device according to the British Hypertension Society protocol. Blood Pressure Monitoring, 13, 49-54. 
Compton, W. M., Conway, K. P., Stinson, F. S., \& Grant, B. F. (2006). Changes in the prevalence of depression and comorbid substance use disorders in the United States between 1991-1992 and 2001-2002. American Journal of Psychiatry, 163, 2141-2147.

Connor, K., \& Miller, J. (2000). Animal-assisted therapy: An in-depth look. Dimensions of Critical Care Nursing, 19(3), 20-26.

Crowley-Robinson, P., \& Blackshaw, J. (1998). Nursing home staffs' empathy for a missing therapy dog, their attitudes to animal-assisted therapy programs and suitable dog breeds. Anthrozoös, 11, 101-104.

Delta Society. (1996). Standards of practice for animal-assisted activities and animalassisted therapy. Renton, WA: Delta Society.

Delta Society. (2010). About animal-assisted activities and animal-assisted therapy. Retrieved from http://www.deltasociety.org/aboutaaat.htm

DeSchriver, M. M., \& Riddick, C. C. (1990). Effects of watching aquariums on elders' stress. Anthrozoos, 4, 44-48.

Dozois, D. A., Dobson, K. S., \& Ahnberg, J. L. (1998). A psychometric evaluation of the Beck Depression Inventory-II. Psychological Assessment, 10(2), 83-89. doi:10.1037/1040-3590.10.2.83

Eddy, J., Hart, L. A., \& Boltz, R. P. (2001). The effects of service dogs on social acknowledgements of people in wheelchairs. Journal of Psychology, 122(1), 3945.

Faul, F., Erdfelder, E., Lang, A.-G., \& Buchner, A. (2007). G*Power 3: A flexible statistical power analysis program for the social, behavioral, and biomedical sciences. Behavior Research Methods, 39, 175-191.

Fine, A. (Ed.). (2006). Handbook on animal-assisted therapy: Theoretical foundations and guidelines for practice ( $2^{\text {nd }}$ ed). San Diego, CA: Academic.

Fine, A. H., \& Mio, J. S. (2006). The future of research, education, and clinical practice in the animal-human bond and animal-assisted therapy: Part C: The role of animal-assisted therapy in clinical practice: The importance of demonstrating empirically oriented psychotherapies. In A. Fine (Ed.), Handbook on animalassisted therapy: Theoretical foundations and guidelines for practice $\left(2^{\text {nd }} \mathrm{ed}\right.$., $\mathrm{pp}$. 513-523). San Diego, CA: Academic.

Folse, E. B., Minder, C. C., Aycock, M. J., \& Santana, R. T. (1994). Animal-assisted therapy and depression in adult college students. Anthrozoös, 7, 188-194. doi:10.2752/089279394787001880 
Friedmann, E. (1983). Social interaction and blood pressure: Influence of animal companions. Journal of Nervous \& Mental Disease, 171, 461-465.

Friedmann, E., Katcher, A. H., Lynch, J. J., \& Thomas, S. A. (1980). Animal companions and one-year survival of patients after discharge from a coronary care unit. Public Health Report, 95, 307-312.

Friedmann, E., \& Thomas, S. A. (1995). Pet ownership, social support, and one-year survival after acute myocardial infarction in the Cardiac Arrhythmia Suppression Trial (CAST). American Journal of Cardiology, 76, 1213-1217.

Friedmann, E., Thomas, S. A., \& Eddy, T. J. (2000). Companion animals and human health: Physical and cardiovascular influences. In A. L. Podberscek, E. S. Paul, \& J. A. Serpell (Eds.), Companion animals and us (pp. 125-142). Cambridge, UK: University Press.

Friedmann, E., \& Tsai, C-C. (2006). The animal-human bond: Health and wellness. In A. Fine (Ed.), Animal-assisted therapy: Theoretical foundations and practice guidelines ( ${ }^{\text {nd }}$ ed., pp. 95-117). San Diego, CA: Academic.

Fydrich, T., Dowdall. D., \& Chambless, D. L. (1992). Reliability and validity of the Beck Anxiety Inventory. Journal of Anxiety Disorders, 6, 55-61.

Garrity. T. F., \& Stallones, L. (1998). Effects of pet contact on human well-being: Review of recent research. In C. Wilson \& D. Turner (Eds.), Companion animals in human health (pp. 3-22). Thousand Oaks, CA: Sage.

Garrity, T., Stallones, L., Marx, M., \& Johnson, T. (1989). Pet ownership and attachment as supportive factors in the health of the elderly. Anthrozoös, 3(1), 35-44.

Geis, B. (1969). Pet-oriented child psychotherapy. Springfield, IL: Charles C. Thomas.

Grossman, P., Niemann, L., Schmidt, S., \& Walach, H. (2004). Mindfulness-based stress reduction and health benefits: A meta-analysis. Journal of Psychosomatic Research, 57(1), 35-43. doi:10.1016/S0022-3999(03)00573-7

Hama, H., Yogo, M., \& Matsuyama, Y. (1996). Effects of stroking horses on both humans' and horses' heart rate responses. Japanese Psychological Research, 38, 66-73.

Hamilton, M. (1959). The assessment of anxiety states by rating. British Journal of Medical Psychology, 32, 50-55.

Hayes, S. C., Strosahl, K., \& Wilson, K. G. (1999). Acceptance and commitment therapy: An experiential approach to behavior change. New York, NY: Guilford. 
Hofmann, S., Sawyer, A., Witt, A., \& Oh, D. (2010). The effect of mindfulness-based therapy on anxiety and depression: A meta-analytic review. Journal of Consulting \& Clinical Psychology, 78(2), 169-183. doi:10.1037/a0018555

Horvath, A., \& Bedi, R. (2002). The therapeutic alliance. In J. C. Norcross (Ed.), Psychotherapy relationships that work: Therapist contributions and responsiveness to patients (pp. 37-69). New York, NY: Oxford University Press.

Howgego, I., Yellowlees, P., Owen, C., Meldrum, L., \& Dark, F. (2003). The therapeutic alliance: The key to effective patient outcome? A descriptive review of the evidence in community mental health case management. Australian \& New Zealand Journal of Psychiatry, 37(2), 169-183.

International Association of Human Animal Interaction Organizations (IAHAIO). (2013). About us. Retrieved from http://www.iahaio.org/

Kabat-Zinn, J. (1990). Full catastrophe living: Using the wisdom of your body and mind to face stress, pain and illness. New York, NY: Delacourt.

Katcher, A. (1983). Man and the living environment: An excursion into cyclical time. In A. Katcher \& A. M. Beck (Eds.), New perspectives on our lives with companion animals (pp. 519-531). Philadelphia, PA: University of Pennsylvania Press.

Katcher, A. (2000). The future of education and research on the animal-human bond and animal-assisted therapy: Part B: Animal-assisted therapy and the study of humananimal relationships: Discipline or bondage? Context or transitional object? In A. H. Fine (Ed.), Handbook on animal-assisted therapy: Theoretical foundations and guidelines for practice (pp. 461-473). San Diego, CA: Academic.

Katcher, A., \& Beck, A. M. (Eds.). (1983). New perspectives on our lives with companion animals. Philadelphia, PA: University of Pennsylvania Press.

Katcher, A., \& Beck, A. (1987). Health and caring for living things. Anthrozoos, 1, 175183.

Katcher, A., Friedmann, E., Beck, A. M., \& Lynch, J. J. (1983). Looking, talking and blood pressure: The physiological consequences of interaction with the living environment. In A. Katcher \& A. M. Beck (Eds.), New perspectives on our lives with companion animals (pp. 351-359). Philadelphia, PA: University of Pennsylvania Press.

Katcher, A., Segal, H., \& Beck, A. (1984). Comparison of contemplation and hypnosis for the reduction of anxiety and discomfort during dental surgery. American Journal of Clinical Hypnosis, 27(1), 14-21. 
Kingston, T., Dooley, B., Bates, A., Lawlor, E., \& Malone, K. (2007). Mindfulness-based cognitive therapy for residual depressive symptoms. Psychology \& Psychotherapy-Theory Research and Practice, 80, 193-203.

Klerman, G. L., \& Weissman, M. M. (1989). Increasing rates of depression. Journal of the American Medical Association, 261, 2229-2234.

Kabat-Zinn, J. (1990). Full catastrophe living: Using the wisdom of your mind to face stress, pain and illness. New York, NY: Dell.

Kristeller, J. L., Baer, R. A., \& Quillian-Wolever, R. (2006). Mindfulness-based approaches to eating disorders. In R. A. Baer (Ed.), Mindfulness-based treatment approaches (pp. 75-91). Burlington, MA: Academic.

Kruger, K., \& Serpell, J. (2006). Animal-assisted interventions in mental health: Definitions and theoretical foundations. A. H. Fine (Ed.), Handbook on animalassisted therapy: Theoretical foundations and guidelines for practice $\left(2^{\text {nd }} \mathrm{ed}\right.$., $\mathrm{pp}$. 21-38). San Diego, CA: Academic.

Lambert, M. J., \& Finch, A. E. (1999). The outcome questionnaire. In M. E. Maruish (Ed.), The use of psychological testing for treatment planning and outcomes assessment ( $2^{\text {nd }}$ ed., pp. 831-869). Mahwah, NJ: Erlbaum.

Lambert. M. J., Whipple, J. L., Hawkins, E. J., Vermeersch, D. A., Nielsen, S. L., \& Smart, D. W. (2003). Is it time for clinicians to routinely track patient outcome? A meta-analysis. Clinical Psychology: Science \& Practice, 10, 288-301.

Lau, M. A., Bishop, S. R., Segal, Z. V., Buis, T., Anderson, N., Carlson, L., ... Carmody, J. (2006). The Toronto Mindfulness Scale: Development and validation. Journal of Clinical Psychology, 62, 1445-1467.

Ledesma, D., \& Kumano, H. (2008). Mindfulness-based stress reduction and cancer: A meta-analysis. Psycho-Oncology, 18, 571-579.

Levinson, B. (1969). Pet-oriented child psychotherapy. Springfield, IL: Charles C Thomas.

Levinson, B. (1972). Pets and human development. Springfield, IL: Charles C Thomas.

Levinson, B. (1984). Human/companion animal therapy. Journal of Contemporary Psychotherapy, 14(2), 131-144.

Levinson, B. (1984). Human/companion animal therapy. Journal of Contemporary Psychotherapy, 14(2), 131-144. 
Linehan, M. M. (1993). Skills training manual for treatment of borderline personality disorder. New York, NY: Guilford.

Locke, J. (1693). Some thoughts concerning education. Retrieved from http://playpen. meraka.csir.co.za/ acdc/education/Dr_Anvind_Gupa/Learners_Library_7_March _2007/Resources/books/John\%20Locke.pdf

Mackenzie, M., Carlson, L., Munoz, M., \& Speca, M. (2007). A qualitative study of selfperceived effects of mindfulness-based stress reduction (MBSR) in a psychosocial oncology setting. Stress and Health: Journal of the International Society for the Investigation of Stress, 23(1), 59-69. doi:10.1002/smi.1120

Mader, B., Hart, L., \& Bergin, B. (1989). Social acknowledgments for children with disabilities: Effects of service dogs. Child Development, 60, 1529-1534.

Marlatt, G. A., \& Gordon, J. R. (1985). Relapse prevention: Maintenance strategies in the treatment of addictive behaviors ( $2^{\text {nd }}$ ed.). New York, NY: Guilford.

Marlatt, G. A., \& Witkiewitz, K. (2005). Relapse prevention for alcohol and drug problems. In G. A. Marlatt \& D. M. Donovan (Eds.), Relapse prevention: Maintenance strategies in the treatment of addictive behaviors ( $2^{\text {nd }}$ ed., pp. 1-44). New York. NY: Guilford.

McCulloch, M. J. (1983). Animal-facilitated therapy: Overview and future direction. In A. H. Katcher \& A. M. Beck (Eds.), New perspectives on our lives with companion animals (pp. 410-426). Philadelphia, PA: University of Pennsylvania Press.

McVarish, C. (1994). The effects of pet facilitated therapy on depressed institutionalized inpatients (Doctoral dissertation). California School of Professional Psychology, Alameda, CA.

Mithen, S. (1999). The hunter-gatherer prehistory of human-animal interactions. Anthrozoös, 12(4), 195-204.

Monti, D. A., Peterson, C., Shakin Kunkel, E. J., Hauck, W. W., Pequignot, E., Rhodes, L., \& Brainard, G. C. (2006). A randomized, controlled trial of mindfulness-based art therapy (MBAT) for women with cancer. Psycho-Oncology, 15, 363-373.

Munsell, K. L., Canfield, M., Templer, D. I., Tangan, K., \& Arikawa, H. (2004). Modification of the Pet Attitude Scale. Society \& Animals, 12(2), 137-142.

Myers, O. E., Jr. (1998). Children and animals: Social development and our connections to other species. Boulder, CO: Westview. 
North American Riding for the Handicapped Association (NARHA). (2010). PATH International. Retrieved from http://narha.org/

Omori, R., \& Hasegawa, T. (2009). The dog with human: Its origins and benefits to modern people. Japanese Journal of Animal Psychology, 59(1), 3-14.

Panzer-Koplow, S. (2000). Effects of animal-assisted therapy on depression and morale among nursing home residents (Doctoral dissertation). Rutgers, The State University of New Jersey, New Brunswick, NJ.

Rossbach, K., \& Wilson, J. (1992). Does a dog's presence make a person appear more likable? Two studies. Anthrozoös, 5(1), 40-51.

Salisbury, J. E. (2010). The beast within: Animals in the middle ages ( $2^{\text {nd }}$ ed.). New York, NY: Routledge.

Segal, Z. V., Williams, J. M. G., \& Teasdale, J. D. (2002). Mindfulness-based cognitive therapy for depression: A new approach for preventing relapse. New York, NY: Guilford.

Serpell, J. A. (1996). In the company of animals: A study of human-animal relationships. New York, NY: Cambridge University Press.

Serpell, J. A. (2000). Creatures of the unconscious: Companion animals as mediators. In A. L. Podberscek, E. S. Paul, \& J. A. Serpell (Eds.), Companion animals and us, (pp. 108-121). Cambridge, UK: Cambridge University Press.

Shapiro, D. (1992). A preliminary study of long-term meditators: Goals, effects, religious orientation, cognitions. Journal of Transpersonal Psychology, 24(1), 23-39.

Shapiro, S., \& Carlson, L. (2009). The art and science of mindfulness: Integrating mindfulness into psychology and the helping professions. Washington, DC: American Psychological Association. doi:10.1037/11885-000.

Shapiro, S., Carlson, L., Astin, J., \& Freedman, B. (2006). Mechanisms of mindfulness. Journal of Clinical Psychology, 62, 373-386. doi:10.1002/jclp.20237.

Siegel, D. (2007). The mindful brain: Reflection and attunement in the cultivation of wellbeing. New York, NY: Norton.

Smeets, G., Merckelbach, H., \& Griez, E. (1996). Panic disorder and right-hemisphere reliance. Anxiety, Stress, \& Coping, 10, 245-255.

Somervill, J. W., Kruglikova, Y. A., Robertson, R. L., Hanson, L. M., \& MacLin, O. H. (2008). Physiological responses by college students to a dog and a cat: Implications for pet therapy. North American Journal of Psychology, 10, 519-528. 
Souter, M. A., \& Miller, M. D. (2007). Do animal assisted activities effectively treat depression: A meta-analysis? Anthrozoos, 20, 167-180.

Spielberger, C. D. (1983). Manual for the State-Trait Anxiety Inventory (Form Y). Palo Alto, CA: Consulting Psychologists Press.

Spielberger, C. D, Gorsuch, R. L., \& Lushene, R. E. (1970). Manual for the State-Trait Anxiety Inventory. Palo Alto, CA: Consulting Psychologists Press.

Staats, S., Wallace, H., \& Anderson, T. (2008). Reasons for companion animal guardianship (pet ownership) from two populations. Society \& Animals, 16, 279291.

Stilgoe, J. R. (2001). Gone barefoot lately? American Journal of Preventative Medicine, 20, 243-244.

Struckus, J. E. (1989). The use of pet-facilitated therapy in the treatment of depression in the elderly: A behavioral conceptualization of treatment effects (Doctoral dissertation). University of Massachusetts, Boston, MA.

Templer, D. I., Salter, C. A., Dickey, S., Baldwin, R., \& Veleber, D. M. (1981). The construction of the Pet Attitude Scale. The Psychological Record, 31, 343-348.

Therapy Dogs International (TDI). (2010). About us. Retrieved from http://tdi-dog.org/

Triebenbacher, S. L. (1998). The relationship between attachment to companion animals and self-esteem: A developmental perspective. In C. C. Wilson \& D. Turner (Eds.), Companion animals in human health (pp. 135-148). Thousand Oaks, CA: Sage.

van der Horst, F., LeRoy, H., \& van der Veer, R. (2008). When strangers meet: John Bowlby and Harry Harlow on attachment behavior. Integrative Psychological \& Behavioral Science, 42, 370-388.

Waldau, P., \& Patton, K. (2006). A communion of subjects: Animals in religion, science, and ethics. New York, NY: Columbia University Press.

Wall, M. J. (1994). The effects of companion animal visitation on mood state and level of speech activity of nursing home residents (Doctoral dissertation). California School of Professional Psychology, San Diego, CA.

Wells, M., \& Perrine, R. (2001). Pets go to college: The influence of pets on students' perceptions of faculty and their offices. Anthrozoös, 14, 161-168.

Wilson, C., \& Turner, D. (1998). Companion animals in human health. Thousand Oaks, CA: Sage. 
Wilson, C. C. (1991). The pet as an anxiolytic intervention. Journal of Nervous and Mental Disease, 179, 482-489.

Wilson, C. C. (2006). Education, and clinical practice in the animal-human bond and animal-assisted therapy: Part B: Human-animal interactions and health: Best evidence and where we go from here. In A. Fine (Ed.), Handbook on animalassisted therapy: Theoretical foundations and guidelines for practice $\left(2^{\text {nd }}\right.$ ed., pp. 499-512). San Diego, CA: Academic Press.

Wilson, E. O. (1984). Biophilia. Cambridge, MA: Harvard University Press. 


\section{APPENDICES}


Appendix A

Intervention Protocol 


\section{Protocol for a Modified Mindfulness-Based Stress Reduction Program for Use With or Without Animal-Assisted Therapy}

\section{Introduction}

The purpose of this intervention protocol is to guide the implementation of a modified mindfulness-based stress reduction program for the treatment of anxiety and depressive symptoms. It has been adapted from the traditional MBSR format to meet the demands of individual therapy and animal-assisted therapy. It is assumed that users of this protocol have a fundamental understanding of the basic theoretical and philosophical basis of MBSR. It is also assumed that they have received some basic training and supervision on the implementation of the treatment. Furthermore, if including AAT, it is assumed that both handler and animal have received training and certification, and that the user is adhering to best practice guidelines as stated by a recognized authority, such as Delta Society.

The main subject of this protocol is a session by session description of mindfulness training. The intervention is designed to be delivered in 6 fifty-minute sessions over the course of 6 weeks, or one session per week. The description of each session includes session objectives, didactic material, and an experiential exercise. Furthermore, specific instructions for implementation in conjunction with animal-assisted therapy are provided for each session. 
Table A1

Outline of Session Timing and Materials

\begin{tabular}{|c|c|c|c|}
\hline Sessions & $\begin{array}{c}\text { Time } \\
\text { (minutes) }\end{array}$ & Activity & Materials \\
\hline \multirow[t]{5}{*}{1} & 30 & $\begin{array}{l}\text { Consent } \\
\text { Physiological assessment (blood pressure, heart rate) } \\
\text { Self-report assessment (FFMQ, PMQ, BAI, BDI-II, } \\
\text { STAI) }\end{array}$ & $\begin{array}{l}\text { Laptop } \\
\text { Blood pressure monitor }\end{array}$ \\
\hline & 15 & Didactic content & \\
\hline & 5 & Physiological assessment (blood pressure, heart rate) & Blood pressure monitor \\
\hline & 15 & Experiential exercise & \\
\hline & 10 & $\begin{array}{l}\text { Physiological assessment (blood pressure, heart rate) } \\
\text { Self-report assessment (S/STAI, TMS, OQ-45, SE) }\end{array}$ & $\begin{array}{l}\text { Laptop } \\
\text { Blood pressure monitor }\end{array}$ \\
\hline \multirow[t]{5}{*}{$2-5$} & 5 & $\begin{array}{l}\text { Physiological assessment (blood pressure, heart rate) } \\
\text { Self-report assessment (S/STAI) }\end{array}$ & $\begin{array}{l}\text { Laptop } \\
\text { Blood pressure monitor }\end{array}$ \\
\hline & 15 & Didactic content & \\
\hline & 5 & Physiological assessment (blood pressure, heart rate) & Blood pressure monitor \\
\hline & 15 & Experiential exercise & Saltines (Session 3) \\
\hline & 10 & $\begin{array}{l}\text { Physiological assessment (blood pressure, heart rate) } \\
\text { Self-report assessment (S/STAI, TMS, OQ- } 45, \text { SE) }\end{array}$ & $\begin{array}{l}\text { Laptop } \\
\text { Blood pressure monitor }\end{array}$ \\
\hline \multirow[t]{5}{*}{6} & 5 & $\begin{array}{l}\text { Physiological assessment (blood pressure, heart rate) } \\
\text { Self-report assessment (S/STAI) }\end{array}$ & $\begin{array}{l}\text { Laptop } \\
\text { Blood pressure monitor }\end{array}$ \\
\hline & 15 & Didactic content & \\
\hline & 5 & Physiological assessment (blood pressure, heart rate) & Blood pressure monitor \\
\hline & 15 & Experiential exercise & \\
\hline & 35 & $\begin{array}{l}\text { Physiological assessment (blood pressure, heart rate) } \\
\text { Self-report assessment (TMS, OQ-45, FFMQ, PMQ, } \\
\text { BAI, BDI-II, STAI, SE, CS) } \\
\text { Compensation for participation }\end{array}$ & $\begin{array}{l}\text { Laptop } \\
\text { Blood pressure monitor }\end{array}$ \\
\hline
\end{tabular}


Table A2

Modified Mindfulness-Based Stress Reduction Treatment Components

\begin{tabular}{lll}
\hline Session & Modified MBSR with AAT & Modified MBSR without AAT \\
\hline 1 Didactic & Benefits of Living Mindfully & Benefits of Living Mindfully \\
Experiential & Breathing (Dog Present) & Breathing \\
2 Didactic & Introduction to Mindfulness & Introduction to Mindfulness \\
Experiential & Body Scan (Dog Present) & Body Scan \\
3 Didactic & Attention & Attention \\
Experiential & Mindfully Petting a Dog & Mindfully Eating a Raisin \\
4 Didactic & Attitude & Attitude \\
Experiential & Stretching (Dog Present) & Stretching \\
5 Didactic & Intention & Intention \\
Experiential & Sitting Meditation (Dog Present) & Sitting Meditation \\
6 Didactic & Review and Termination & Review and Termination \\
Experiential & Lovingkindness Meditation (Dog Present) & Lovingkindness Meditation \\
\hline
\end{tabular}




\section{Session 1 \\ Benefits of Living Mindfully}

\section{Session Structure}

The session will begin by obtaining consent and answering any questions the client may have regarding the research process. The client will complete FFMQ, PMQ, BAI, BDI-II, and STAI on the laptop. Blood pressure and heart rate will be assessed. The therapist will then teach the didactic content. Blood pressure and heart rate will be assessed. The therapist will then guide the experiential exercise. Blood pressure and heart rate will be assessed. The client will complete S/STAI, TMS, OQ-45, and SE on the laptop.

\section{Objectives}

The objectives of this session are to explore the idea of "mindlessness" and discuss the potential benefits of living mindfully.

\section{Didactic Content (15 minutes)}

Introduce therapist. Allow the client introduce him/herself and invite him/her to ask questions regarding the intervention process. Begin by asking the client to explore his/her current distress and how he/she would like to live differently. Introduce the concept of "mindlessness," how it can affect the way one experiences life, and ways in which the client is currently living mindlessly. Provide examples of thoughts, emotions, and behaviors that are mindless and then those that are mindful. Invite the client to consider how life would be different if he/she lived in the moment as opposed to the past or future.

\section{Experiential Exercise (15 minutes)}

Breathing: Invite the client to practice mindful breathing. Start by having the client center his/her mind by focusing on taking three natural breaths. Have the client sit in a comfortable position. Encourage the client to maintain his/her attention on the breath, noticing the body's sensations with each inhale and exhale. Guide the exercise for 5 minutes, spend 5 minutes discussing the client's experience with the exercise, and then do the exercise again for 5 minutes, providing less verbal guidance as time elapses.

\section{Animal-Assisted Therapy Modifications}

The dog is present in the room. Introduce the therapy dog and state the rules for interacting with the dog. Rules include: 1) Demonstrate kindness and respect. The client is directed to be friendly, not attempt to startle or harm the animal, and allow the animal personal space if desired. 2) Promote safety of yourself and others. The animal will be removed from the room at the request of the client or based on the judgment of the therapist.

Allow the client to interact freely with the therapy dog throughout the session. Encourage the dog to sit near the client during didactic learning and discussions, while also encouraging the client to pet the dog. Use the dog as a verbal example and physical model of mindfulness during didactic learning. At the start of the experiential exercise, have the client center his/her mind by petting the dog slowly three times while taking three natural breaths.

\section{Homework}

Encourage the client to practice the experiential exercise throughout the following week. If the client worked with the therapy dog, explain how the client can practice the exercise without a dog, following the protocol guidelines for this session. 


\section{Session 2 Introduction to Mindfulness}

\section{Session Structure}

The client will complete S-STAI on the laptop. Blood pressure and heart rate will be assessed. The therapist will then teach the didactic content. Blood pressure and heart rate will be assessed. The therapist will then guide the experiential exercise. Blood pressure and heart rate will be assessed. The client will complete S/STAI, TMS, OQ-45, and SE on the laptop.

\section{Objectives}

The objectives of this session are to introduce the client to mindfulness and its core elements of attention, attitude, and intention, including how each affects and builds off the other.

\section{Didactic Content (15 minutes)}

Begin by encouraging the client to further explore the differences between mindlessness and mindfulness, providing labels to the elements of attention, attitude, and intention as aspects of each are discussed. It may be helpful to note that attention is like "what," attitude is like "how," and intention is like "why." Discuss the process of being mindful and how each element evolves in relation to the other. Talk about both formal and informal ways to practice, providing examples of each and noting that mindfulness is a skill that takes time to develop.

\section{Experiential Exercise (15 minutes)}

Body Scan: Invite the client to lie in a comfortable position on his/her back on the couch. Start by having the client center his/her mind by focusing on taking three natural breaths. Have the client direct his/her attention to each part of the body, moving slowly from the toes upward. Verbally guide the client to move his/her mind through each region of the body, encouraging the client to feel each part of the body. Ask the client to breathe in to and out from each region then let go of that part as his/her attention is guided to the next. As the body scan is completed, allow the client to exist in silence and stillness before guiding him/her to gently reorient him/herself by moving hands and feet and opening eyes.

\section{Animal-Assisted Therapy Modifications}

The dog is present in the room. Encourage the dog and client to greet at the beginning of the session. The client is allowed to interact freely throughout the session with the therapy dog. Encourage the dog to sit near the client during didactic learning and discussions, while also encouraging the client to pet the dog. Use the dog as a verbal example and physical model of mindfulness during didactic learning. At the start of the experiential exercise, have the client center his/her mind by petting the dog slowly three times while taking three natural breaths.

\section{Homework}

Encourage the client to practice the experiential exercise throughout the following week. If the client worked with the therapy dog, explain how the client can practice the exercise without a dog, following the protocol guidelines for this session. 


\section{Session 3 \\ Attention}

\section{Session Structure}

The client will complete S-STAI on the laptop. Blood pressure and heart rate will be assessed. The therapist will then teach the didactic content. Blood pressure and heart rate will be assessed. The therapist will then guide the experiential exercise. Blood pressure and heart rate will be assessed. The client will complete S/STAI, TMS, OQ-45, and SE on the laptop.

\section{Objectives}

The objectives of this session are to focus on the core element of attention to the present moment, including one's internal and external experiences.

\section{Didactic Content (15 minutes)}

Begin by encouraging the client to recount various events of the previous day, noting if there were times he/she was or was not "in the moment." Help the client differentiate between these experiences by providing examples of situations that typically lead to one or the other, such as mindlessly eating or mindfully playing a sport. Have the client explore the reasons for engaging in that manner as well as the pros and cons to both types of experiences. Invite the client to think about how practicing attention may impact his/her life.

\section{Experiential Exercise (15 minutes)}

Mindfully Eating a Raisin: Invite the client to hold a few raisins in his/her hand. Start by having the client center his/her mind by focusing on taking three natural breaths. Encourage the client to explore the raisin with all five senses and verbally describe his/her observations throughout the exercise. Guide the client to first look at the raisin in the hand and while holding it up to the light, then touch the raisin with varying pressure, then smell the raisin, and then listen to the raisin by holding it up to the ear and rolling it between two fingers. Have the client slowly place the raisin between his/her lips. Instruct the client to notice his/her reactions at that moment and while slowly moving the raisin into the mouth, chewing, and swallowing. At the end, discuss the client's experience of giving attention to the raisin and the process of eating, noting any challenges or insights the client experienced.

\section{Animal-Assisted Therapy Modifications}

The dog is present in the room. Encourage the dog and client to greet at the beginning of the session. The client is allowed to interact freely throughout the session with the therapy dog. Encourage the dog to sit near the client during didactic learning and discussions, while also encouraging the client to pet the dog. Use the dog as a verbal example and physical model of mindfulness during didactic learning. At the start of the experiential exercise, have the client center his/her mind by petting the dog slowly three times while taking three natural breaths. Replace the exercise "Mindfully Eating a Raisin" with "Mindfully Petting a Dog."

Mindfully Petting a Dog: Invite the client to sit next to the dog. Encourage the client to interact with the dog with all five senses and verbally describe his/her observations throughout the exercise. Guide the client to first look at the dog, then touch (not pet) the dog, then smell the dog, and then listen to the dog. Have the client slowly place his/her hand just above the dog's fur. Instruct the client to notice his/her reactions at that moment and while slowly petting the dog. At the end, discuss the client's experience of giving attention to the dog and the process of petting, noting any challenges or insights the client experienced.

\section{Homework}

Encourage the client to practice the experiential exercise throughout the following week. If the client worked with the therapy dog, explain how the client can practice the exercise without a dog, following the protocol guidelines for this session. 


\section{Session 4 \\ Attitude}

\section{Session Structure}

The client will complete S-STAI on the laptop. Blood pressure and heart rate will be assessed. The therapist will then teach the didactic content. Blood pressure and heart rate will be assessed. The therapist will then guide the experiential exercise. Blood pressure and heart rate will be assessed. The client will complete S/STAI, TMS, OQ-45, and SQ on the laptop.

\section{Objectives}

The objectives of this session are to focus on the core element of attitude, including non-judgment and kindness.

\section{Didactic Content (15 minutes)}

Begin by asking the client to examine two situations that have similar physical experiences but different emotional or cognitive experiences (examples could include pain from exercise or from surgery, or heart rate increase from excitation or from fear). Discuss the differences between the two situations, noting that we often judge things as good or bad and then choose to experience or avoid based on those reactions. Invite the client to again think about the "negative" experience, imagining him/herself being open and welcoming to that specific aspect. Discuss the potential benefits of approaching experiences and the self with kindness, acceptance, and curiosity.

\section{Experiential Exercise (15 minutes)}

Stretching: Invite the client to "play" by engaging in basic standing stretches, encouraging attention to the present moment and a nonjudgmental attitude. Start by having the client center his/her mind by focusing on taking three natural breaths. While the client is standing, have him/her stretch each arm up one at a time, then up together, then out to the sides. Direct the client to drop his/her hands to the hips and then twist from the torso, trying to turn as much as possible, and then relax all muscles but the necessary ones to stay turned. Do the same for the opposite side. Have the client stand straight again and do gentle head rolls then shoulder rolls in both directions. Finally, have the client reach both arms up then sweep them down to the ground while bending at the waist. Throughout the exercise, encourage the client to simply notice sensations, thoughts, and feelings without judgment.

\section{Animal-Assisted Therapy Modifications}

The dog is present in the room. Encourage the dog and client to greet at the beginning of the session. The client is allowed to interact freely throughout the session with the therapy dog. Encourage the dog to sit near the client during didactic learning and discussions, while also encouraging the client to pet the dog. Use the dog as a verbal example and physical model of mindfulness during didactic learning. At the start of the experiential exercise, have the client center his/her mind by petting the dog slowly three times while taking three natural breaths. While stretching, prompt the client to reach out or down toward the dog, touching the dog if possible.

\section{Homework}

Encourage the client to practice the experiential exercise throughout the following week. If the client worked with the therapy dog, explain how the client can practice the exercise without a dog, following the protocol guidelines for this session. 


\section{Session 5 Intention}

\section{Session Structure}

The client will complete S-STAI on the laptop. Blood pressure and heart rate will be assessed. The therapist will then teach the didactic content. Blood pressure and heart rate will be assessed. The therapist will then guide the experiential exercise. Blood pressure and heart rate will be assessed. The client will complete S/STAI, TMS, OQ-45, and SE on the laptop.

\section{Objectives}

The objectives of this session are to focus on the core element of intention, including one's personal values.

\section{Didactic Content (15 minutes)}

Begin by discussing with the client what motivates him/her to do various daily activities, noting why these motivations exist. Then, work with the client to create a list of reasons to practice mindfulness. Discuss the idea that the intention behind mindfulness practice often changes over time and note any judgment of specific intentions. Talk about how intention interacts with the other elements of mindfulness, attitude, and attention, allowing the client to explore these interconnections.

\section{Experiential Exercise (15 minutes)}

Sitting Meditation: Invite the client to sit in a comfortable position. Start by having the client center his/her mind by focusing on taking three natural breaths. Direct the client to sit still and observe the breath as it flows in and out, noting if attention has moved elsewhere and gently redirecting it back to the breath. Emphasize the need to engage in the exercise with patience and kindness to the self. Encourage the client to resist the impulse to shift position in reaction to bodily discomfort and instead direct his/her attention to the sensations and mentally welcome them. If the client's attention is relatively stable on the breath, encourage $\mathrm{him} / \mathrm{h}$ er to shift attention to the process of thinking. Note that the goal is not to make one's mind blank but to be aware of various thoughts and feelings as they arise and how one handles them.

\section{Animal-Assisted Therapy Modifications}

The dog is present in the room. Encourage the dog and client to greet at the beginning of the session. The client is allowed to interact freely throughout the session with the therapy dog. Encourage the dog to sit near the client during didactic learning and discussions, while also encouraging the client to pet the dog. Use the dog as a verbal example and physical model of mindfulness during didactic learning. At the start of the experiential exercise, have the client center his/her mind by petting the dog slowly three times while taking three natural breaths.

\section{Homework}

Encourage the client to practice the experiential exercise throughout the following week. If the client worked with the therapy dog, explain how the client can practice the exercise without a dog, following the protocol guidelines for this session. 


\section{Session 6 Review and Termination}

\section{Session Structure}

The client will complete S-STAI on the laptop. Blood pressure and heart rate will be assessed. The therapist will then teach the didactic content. Blood pressure and heart rate will be assessed. The therapist will then guide the experiential exercise. Blood pressure and heart rate will be assessed. The client will complete STAI, TMS, OQ-45, FFMQ, PMQ, BAI, BDI-II, SE, and CS on the laptop. The therapist will provide compensation for participation.

\section{Objectives}

The objectives of this session are to review previous learned information and skills, as well as provide closure before completion of the intervention.

\section{Didactic Content (15 minutes)}

Begin by reviewing the previously learned information, including the three main elements of mindfulness and how they interact within the process. Also review each exercise included in the intervention and discuss the use of informal practice. Work with the client to determine how he/she can continue to practice mindfulness in his/her daily life if desired. Conclude by thanking the client for his/her willingness to engage in the experience and offering the client a chance to respond to the experience.

\section{Experiential Exercise (15 minutes)}

Lovingkindness meditation: Invite the client to sit comfortably and begin by stabilizing and calming the mind through mindful breathing. Start by having the client center his/her mind by focusing on taking three natural breaths. Then guide the client to consciously invoke feelings of love and kindness towards him/herself, perhaps by silently saying statements such as "May I be filled with compassion; may I feel kindness toward myself." Next the client will invoke these feelings towards someone he/she cares about, while visualizing the person receiving the well-wishes. After thinking of multiple people, the client will then focus his/her energy on someone for whom he/she may have a more difficult time feeling compassion. After this practice, guide the client to return to the breath and notice the experience of feeling generous and loving towards others.

\section{Animal-Assisted Therapy Modifications}

The dog is present in the room. Encourage the dog and client to greet at the beginning of the session. The client is allowed to interact freely throughout the session with the therapy dog. Encourage the dog to sit near the client during didactic learning and discussions, while also encouraging the client to pet the dog. Use the dog as a verbal example and physical model of mindfulness during didactic learning. At the start of the experiential exercise, have the client center his/her mind by petting the dog slowly three times while taking three natural breaths. Provide an opportunity for the client to say goodbye to the dog before the end of the final session.

\section{Homework}

Encourage the client to practice the experiential exercise throughout the following week. If the client worked with the therapy dog, explain how the client can practice the exercise without a dog, following the protocol guidelines for this session. 
Appendix B

Measures 
1. What is your sex?

\section{Background Information}

$1=$ female

$2=$ male

2. What is your marital status?

$1=$ single

$2=$ married

$3=$ divorced

$4=$ separated

$5=$ remarried

$6=$ widowed

$7=$ cohabitating

3. What is your birthdate? (month/day/year)

4. How many years of post-high school education have you completed?

5. What is your ethnicity/race?

$1=$ African American

2=Asian American

$3=$ Caucasian

4=Hispanic/Latino

$5=$ Native American

$6=$ Multiethnic/Multiracial

$7=$ Other

6. Are you currently in therapy or receiving any psychological services? If yes, what? $1=$ no

$2=$ yes

7. Are you currently taking any medications? If yes, what?

$1=$ no

$2=$ yes

8. Are you allergic to dogs?

$1=$ no

$2=$ yes

9. Do you have any physical or medical complications that would limit your ability to participate in light exercise?

$1=$ no

$2=$ yes 
10. Have you had any previous training in mindfulness? If yes, what?

$1=$ no

$2=$ yes

11. How often do you practice mindfulness?

$0=$ never or rarely

$1=$ occasionally

$2=$ weekly

$3=$ daily

12. Rate your level of expertise in mindfulness on a scale of 1 to 10 , with 1 meaning no expertise and 10 meaning complete expertise.

13. Are you experiencing symptoms of distress, anxiety, and/or depression that you would like to learn skills to decrease? If yes, please rate the level of negative impact that your distress has on your life on a 1 to 10 scale (1 means no negative impact, 10 means extreme negative impact).

$0=$ no

$1-10$ (select one number) 


\section{$\underline{\text { Pet Attitude Scale - Modified }}$}

Please answer each of the following questions as honestly as you can, in terms of how you feel right now. This questionnaire is anonymous and no one will ever know which answers are yours. So, don't worry about how you think others might answer these questions. There isn't any right or wrong answers. All that matters is that you express your true thoughts on the subject. Please answer by circling one of the following seven numbers for each question.

$\begin{array}{ccccccc}1 & 2 & 3 & 4 & 5 & 6 & 7 \\ \text { Strongly } & \text { Moderately } & \text { Slightly } & \text { Unsure } & \text { Slightly } & \text { Moderately } & \text { Strongly } \\ \text { Disagree } & \text { Disagree } & \text { Disagree } & & \text { Agree } & \text { Agree } & \text { Agree }\end{array}$

1. I really like seeing pets enjoy their food.

2. My pet means more to me than any of my friends (or would if I had one).

3. I would like to have a pet in my home.

4. Having pets is a waste of money.

5. House pets add happiness to my life (or would if I had one).

6. I feel that pets should always be kept outside.

7. I spend time every day playing with my pet (or would if I had one).

8. I have occasionally communicated with my pet and understood what it was trying to express (or would if I had one).

9. The world would be a better place if people would stop spending so much time caring for their pets and started caring more for other human beings instead.

10. I like to feed animals out of my hand.

11. I love pets.

12. Animals belong in the wild or in zoos, but not in the home.

13. If you keep pets in the house you can expect a lot of damage to furniture.

14. I like house pets.

15. Pets are fun but it's not worth the trouble of owning one.

16. I frequently talk to my pets (or would if I had one).

17. I hate animals.

18. You should treat your house pets with as much respect as you would a human member of your family.

*Reverse score items 4, 6, 9, 12, 13, 15, and 17. 


\section{Five Facet Mindfulness Questionnaire}

Please rate each of the following statements using the scale provided. Enter the number in the blank that best describes your own opinion of what is generally true for you.

$\begin{array}{ccccc}1 & 2 & 3 & 4 & 5 \\ \text { never or very } & \text { rarely } & \text { sometimes } & \text { often } & \text { very often or } \\ \text { rarely true } & \text { true } & \text { true } & \text { true } & \text { always true }\end{array}$

1. When I'm walking, I deliberately notice the sensations of my body moving.

2. I'm good at finding words to describe my feelings.

3. I criticize myself for having irrational or inappropriate emotions.

4. I perceive my feelings and emotions without having to react to them.

5. When I do things, my mind wanders off and I'm easily distracted.

6. When I take a shower or bath, I stay alert to the sensations of water on my body.

7. I can easily put my beliefs, opinions, and expectations into words.

8. I don't pay attention to what I'm doing because I'm daydreaming, worrying, or otherwise distracted.

9. I watch my feelings without getting lost in them.

10. I tell myself I shouldn't be feeling the way I'm feeling.

11. I notice how foods and drinks affect my thoughts, bodily sensations, and emotions.

12 It's hard for me to find the words to describe what I'm thinking.

13 I am easily distracted.

14 I believe some of my thoughts are abnormal or bad and I shouldn't think that way.

15 I pay attention to sensations, such as the wind in my hair or sun on my face.

16. I have trouble thinking of the right words to express how I feel about things.

17. I make judgments about whether my thoughts are good or bad.

18. I find it difficult to stay focused on what's happening in the present.

19. When I have distressing thoughts or images, I "step back" and am aware of the thought or image without getting taken over by it.

20. I pay attention to sounds, such as clocks ticking, birds chirping, or cars passing.

21. In difficult situations, I can pause without immediately reacting.

22. When I have a sensation in my body, it's difficult for me to describe it because I can't find the right words.

23. It seems I am "running on automatic" without much awareness of what I'm doing.

24. When I have distressing thoughts or images, I feel calm soon after.

25. I tell myself that I shouldn't be thinking the way I'm thinking.

26. I notice the smells and aromas of things. 
27. Even when I'm feeling terribly upset, I can find a way to put it into words.

28. I rush through activities without being really attentive to them.

29. When I have distressing thoughts or images I am able just to notice them without reacting.

30. I think some of my emotions are bad or inappropriate and I shouldn't feel them.

31. I notice visual elements in art or nature, such as colors, shapes, textures, or patterns of light and shadow.

32. My natural tendency is to put my experiences into words.

33. When I have distressing thoughts or images, I just notice them and let them go.

34. I do jobs or tasks automatically without being aware of what I'm doing.

35. When I have distressing thoughts or images, I judge myself as good or bad, depending what the thought/image is about.

36. I pay attention to how my emotions affect my thoughts and behavior.

37. I can usually describe how I feel at the moment in considerable detail.

38. I find myself doing things without paying attention.

39. I disapprove of myself when I have irrational ideas.

\section{Scoring Information:}

\section{Observe items:}

$1,6,11,15,20,26,31,36$

Describe items:

2, 7, 12R, 16R, 22R, 27, 32, 37

Act with Awareness items:

5R, 8R, 13R, 18R, 23R, 28R, 34R, 38R

Nonjudge items:

3R, 10R, 14R, 17R, 25R, 30R, 35R, 39R

Nonreact items:

$4,9,19,21,24,29,33$ 


\section{Philadelphia Mindfulness Questionnaire}

For each of the statements located below, please indicate your response by entering the number in the blank next to each statement. Use the scale shown below:

$\begin{array}{ccccc}1 & 2 & 3 & 4 & 5 \\ \text { Never } & \text { Rarely } & \text { Sometimes } & \text { Often } & \text { Very Often }\end{array}$

1. I am aware of what thoughts are passing through my mind.*

2. I try to distract myself when I feel unpleasant emotions. $\dagger$

3. When talking with other people, I am aware of their facial and body expressions.*

4. There are aspects of myself I don't want to think about. $\uparrow$

5. When I shower, I am aware of how the water is running over my body.*

6. I try to stay busy to keep thoughts or feelings from coming to mind. $\dagger$

7. When I am startled, I notice what is going on inside my body.*

8. I wish I could control my emotions more easily.†

9. When I walk outside, I am aware of smells or how the air feels against my face.*

10. I tell myself that I shouldn't have certain thoughts. $\dagger$

11. When someone asks how I am feeling, I can identify my emotions easily.*

12. There are things I try not to think about. $\dagger$

13. I am aware of thoughts I'm having when my mood changes.*

14. I tell myself that I shouldn’t feel sad.†

15. I notice changes inside my body, like my heart beating faster or my muscles getting tense.*

16. If there is something I don't want to think about, I'll try many things to get it out of my mind. $\dagger$

17. Whenever my emotions change, I am conscious of them immediately.*

18. I try to put my problems out of my mind. $\dagger$

19. When talking with other people, I am aware of the emotions I am experiencing.*

20. When I have a bad memory, I try to distract myself to make it go away. $\dagger$

*Awareness subscale item. Higher scores indicate higher levels of awareness.

$\dagger$ Acceptance subscale item. Items 2, 4, 6, 8, 10, 12, 14, 16, 18, 20 are reversed scored. Higher scores indicate greater acceptance. 


\section{Toronto Mindfulness Scale}

We are interested in what you just experienced. Below is a list of things that people sometimes experience. Please read each statement. Next to each statement are five choices: "not at all," "a little," "moderately," "quite a bit," and "very much." Please indicate the extent to which you agree with each statement. In other words, how well does the statement describe what you just experienced, just now?

$0=$ Not at all

$1=$ A little

$2=$ Moderately

$3=$ Quite a bit

$4=$ Very much

1. I experienced myself as separate from my changing thoughts and feelings.

2. I was more concerned with being open to my experiences than controlling or changing them.

3. I was curious about what I might learn about myself by taking notice of how I react to certain thoughts, feelings, or sensations.

4. I experienced my thoughts more as events in my mind than as a necessarily accurate reflection of the way things 'really' are.

5. I was curious to see what my mind was up to from moment to moment.

6. I was curious about each of the thoughts and feelings that I was having.

7. I was receptive to observing unpleasant thoughts and feelings without interfering with them.

8. I was more invested in just watching my experiences as they arose, than in figuring out what they could mean.

9. I approached each experience by trying to accept it, no matter whether it was pleasant or unpleasant.

10. I remained curious about the nature of each experience as it arose.

11. I was aware of my thoughts and feelings without over-identifying with them.

12. I was curious about my reactions to things.

13. I was curious about what I might learn about myself by just taking notice of what my attention gets drawn to.

Curiosity score: $3,5,6,10,12,13$

Decentering score: $1,2,4,7,8,9,11$ 


\section{Session Engagement}

1. How many times did you practice mindfulness in the past week?

Please answer the following questions on a scale of 1 to 10 , with 1 indicating "not at all" and 10 indicating "extremely."

2. How engaged were you in this session?

3. How much are you looking forward to attending the next session?

4. How mindful were you during this session? 


\section{Client Satisfaction}

Please provide us with feedback about the training you completed. We are interested in your honest opinions. Please answer all of the questions. Thank you; we appreciate your help!

1. Did you enjoy participating in this training?

$4=$ Yes, very much

$3=$ Yes, somewhat

$2=$ Yes, a little

$1=$ Not at all

2. How effective was the therapist in facilitating the training?

$4=$ Very effective

$3=$ Somewhat effective

$2=$ A little effective

$1=$ Not at all effective

3. How likely are you to use the skills you learned in the future?

$4=$ Very likely

$3=$ Somewhat likely

$2=$ A little likely

$1=$ Not at all likely

4. Would you recommend a training like this to friends or family?

$4=$ Yes, definitely

$3=$ Yes, I think so

$2=$ No, I don't think so

$1=$ No, definitely not

5. Please rate your overall impression of your interactions with the therapist.

$4=$ Excellent

$3=$ Good

$2=$ Fair

$1=$ Poor

6. Has participating in this training helped you to deal more effectively with your problems?

$4=$ Yes, it helped a great deal

$3=$ Yes, it helped somewhat

$2=$ No, it really didn't help

$1=$ No, it seemed to make things worse 
7. In an overall, general sense, how satisfied are you with the training you have received?

$4=$ Very satisfied

$3=$ Mostly satisfied

$2=$ Indifferent or mildly dissatisfied

$1=$ Quite dissatisfied

8. If you were to seek help in the future, would you participate in a similar intervention?

$4=$ Yes, definitely

$3=$ Yes, I think so

$2=$ No, I don't think so

$1=$ No, definitely not

9. What aspects of the training did you find most helpful and/or enjoyable?

10. What would you change about this experience?

Comments and Suggestions:

Please answer the following questions if you worked with a therapy dog.

11. Would you have completed this training if there was no therapy dog?

$4=$ Yes, definitely

$3=$ Yes, I think so

$2=$ No, I don't think so

$1=$ No, definitely not

12. Did the therapy dog make this experience more helpful and/or enjoyable?

$4=$ Yes, definitely

$3=$ Yes, I think so

$2=$ No, I don't think so

$1=$ No, definitely not 
Appendix C

Descriptive Statistics and Correlation Tables 
Table C1

Descriptive Statistics for the BDI-II, BAI, STAI-Trait, FFMQ, and PHLMS

\begin{tabular}{|c|c|c|c|c|c|c|c|c|c|}
\hline \multirow[b]{2}{*}{ Variable } & & \multicolumn{4}{|c|}{ Pretreatment } & \multicolumn{4}{|c|}{ Posttreatment } \\
\hline & & Mean & $S D$ & Kurtosis & Skewness & Mean & $S D$ & Kurtosis & Skewness \\
\hline \multirow[t]{3}{*}{ BDI-II } & MBSR & 18.45 & 9.21 & 3.29 & 1.32 & 10.82 & 10.05 & .04 & .89 \\
\hline & $\mathrm{MBSR}+\mathrm{AAT}$ & 24.20 & 12.12 & -1.21 & -.42 & 10.00 & 6.78 & -.52 & .70 \\
\hline & Total & 21.19 & 10.84 & -.77 & .35 & 10.43 & 8.45 & .22 & .88 \\
\hline \multirow[t]{3}{*}{ BAI } & MBSR & 18.45 & 5.73 & .68 & -.43 & 12.27 & 8.19 & -1.64 & .09 \\
\hline & $\mathrm{MBSR}+\mathrm{AAT}$ & 18.00 & 9.104 & -.37 & .63 & 11.70 & 3.56 & -1.66 & .03 \\
\hline & Total & 18.24 & 7.33 & -.01 & .34 & 12.00 & 6.27 & -.68 & .20 \\
\hline \multirow[t]{3}{*}{ STAI-trait } & MBSR & 53.82 & 9.75 & .41 & .68 & 42.82 & 13.21 & .50 & .68 \\
\hline & $\mathrm{MBSR}+\mathrm{AAT}$ & 57.80 & 8.26 & .60 & -.68 & 42.70 & 8.70 & -.37 & .73 \\
\hline & Total & 55.71 & 9.07 & -.47 & .06 & 42.76 & 11.01 & .54 & .68 \\
\hline \multicolumn{10}{|l|}{ FFMQ } \\
\hline \multirow[t]{3}{*}{ Observing } & MBSR & 22.36 & 5.41 & .27 & .43 & 28.00 & 6.08 & -1.63 & -.16 \\
\hline & $\mathrm{MBSR}+\mathrm{AAT}$ & 21.30 & 6.36 & -.42 & -.43 & 27.00 & 7.36 & .47 & -.23 \\
\hline & Total & 21.86 & 5.76 & -.18 & -.12 & 27.52 & 6.57 & -.40 & -.23 \\
\hline \multirow[t]{3}{*}{ Describing } & MBSR & 20.18 & 7.13 & -1.37 & .51 & 26.64 & 6.17 & -1.43 & -.38 \\
\hline & $\mathrm{MBSR}+\mathrm{AAT}$ & 23.30 & 6.60 & -.44 & .79 & 28.80 & 5.20 & -1.28 & -.46 \\
\hline & Total & 21.67 & 6.90 & -.87 & .47 & 27.67 & 5.70 & -1.12 & -.46 \\
\hline \multirow[t]{3}{*}{ Act aware } & MBSR & 22.91 & 3.21 & -.51 & -.97 & 31.18 & 8.52 & -.93 & .05 \\
\hline & $\mathrm{MBSR}+\mathrm{AAT}$ & 22.80 & 6.18 & -.26 & -.84 & 32.30 & 6.65 & 1.41 & -1.30 \\
\hline & Total & 22.86 & 4.73 & .51 & -.90 & 31.71 & 7.52 & -.55 & -.39 \\
\hline \multirow[t]{3}{*}{ Nonjudging } & MBSR & 22.00 & 5.62 & -.52 & .33 & 30.27 & 4.78 & -.46 & .37 \\
\hline & $\mathrm{MBSR}+\mathrm{AAT}$ & 18.20 & 2.61 & -.03 & -.83 & 27.20 & 8.30 & -1.66 & -.30 \\
\hline & Total & 20.19 & 4.76 & .84 & .87 & 28.81 & 6.70 & -.45 & -.53 \\
\hline \multirow[t]{3}{*}{ Nonreacting } & MBSR & 17.73 & 4.34 & -1.00 & .20 & 23.18 & 4.64 & -.27 & -.49 \\
\hline & $\mathrm{MBSR}+\mathrm{AAT}$ & 17.30 & 4.32 & 1.53 & -1.01 & 21.50 & 5.74 & 2.42 & .89 \\
\hline & Total & 17.52 & 4.23 & -.07 & -.34 & 22.38 & 5.13 & .39 & .22 \\
\hline \multicolumn{10}{|l|}{ PHLMS } \\
\hline \multirow[t]{3}{*}{ Awareness } & MBSR & 28.18 & 6.01 & 1.23 & -.19 & 35.09 & 8.44 & -.06 & -.69 \\
\hline & $\mathrm{MBSR}+\mathrm{AAT}$ & 28.70 & 7.56 & -.61 & -.14 & 35.60 & 6.33 & .40 & -.87 \\
\hline & Total & 28.43 & 6.62 & -.20 & -.12 & 35.33 & 7.33 & .02 & -.73 \\
\hline \multirow[t]{3}{*}{ Acceptance } & MBSR & 23.82 & 7.86 & -1.86 & .13 & 33.73 & 7.23 & .72 & .69 \\
\hline & $\mathrm{MBSR}+\mathrm{AAT}$ & 22.50 & 7.18 & -.84 & -.24 & 29.60 & 5.99 & -.86 & .30 \\
\hline & Total & 23.19 & 7.39 & -1.32 & .01 & 31.76 & 6.83 & .44 & .62 \\
\hline
\end{tabular}


Table C2

Descriptive Statistics for the OQ-45

\begin{tabular}{|c|c|c|c|c|c|}
\hline \multicolumn{2}{|l|}{ Variable } & Mean & $S D$ & Kurtosis & Skewness \\
\hline \multicolumn{6}{|l|}{ OQ-45 } \\
\hline \multirow[t]{3}{*}{ Session 1} & MBSR & 80.82 & 16.42 & -.60 & .54 \\
\hline & $\mathrm{MBSR}+\mathrm{AAT}$ & 87.50 & 18.36 & .85 & -.67 \\
\hline & Total & 84.00 & 17.27 & -.59 & -.05 \\
\hline \multirow[t]{3}{*}{ Session 2} & MBSR & 71.18 & 21.84 & -1.26 & .21 \\
\hline & $\mathrm{MBSR}+\mathrm{AAT}$ & 75.20 & 16.78 & -1.57 & -.15 \\
\hline & Total & 73.10 & 19.22 & -1.23 & .01 \\
\hline \multirow[t]{3}{*}{ Session 3} & MBSR & 66.80 & 26.08 & -.46 & .81 \\
\hline & $\mathrm{MBSR}+\mathrm{AAT}$ & 68.00 & 20.16 & 1.07 & -.18 \\
\hline & Total & 67.37 & 22.83 & -.30 & .47 \\
\hline \multirow[t]{3}{*}{ Session 4} & MBSR & 64.64 & 25.04 & .07 & .68 \\
\hline & $\mathrm{MBSR}+\mathrm{AAT}$ & 66.40 & 18.67 & -.55 & -.01 \\
\hline & Total & 65.48 & 21.71 & -.18 & .43 \\
\hline \multirow[t]{3}{*}{ Session 5} & MBSR & 59.91 & 24.29 & -.09 & -.06 \\
\hline & $\mathrm{MBSR}+\mathrm{AAT}$ & 60.60 & 13.62 & 3.31 & 1.52 \\
\hline & Total & 60.24 & 19.46 & .73 & .13 \\
\hline \multirow[t]{3}{*}{ Session 6} & MBSR & 52.27 & 24.37 & -1.18 & -.32 \\
\hline & $\mathrm{MBSR}+\mathrm{AAT}$ & 53.80 & 18.35 & 1.69 & 1.10 \\
\hline & Total & 53.00 & 21.19 & -.43 & .03 \\
\hline
\end{tabular}


Table C3

Descriptive Statistics for the A-State

\begin{tabular}{|c|c|c|c|c|c|c|c|c|c|}
\hline \multirow[b]{2}{*}{ STAI-state } & & \multicolumn{4}{|c|}{ Presession } & \multicolumn{4}{|c|}{ Postsession } \\
\hline & & Mean & $S D$ & Kurtosis & Skewness & Mean & $S D$ & Kurtosis & Skewness \\
\hline \multirow[t]{3}{*}{ Session 1} & MBSR & 50.64 & 13.25 & -.35 & .58 & 38.36 & 8.81 & -.45 & .91 \\
\hline & $\mathrm{MBSR}+\mathrm{AAT}$ & 53.70 & 11.25 & -1.34 & -.62 & 41.60 & 8.87 & -1.86 & .26 \\
\hline & Total & 52.10 & 12.13 & -1.02 & .07 & 39.90 & 8.77 & -1.30 & .53 \\
\hline \multirow[t]{3}{*}{ Session 2} & MBSR & 44.64 & 13.12 & 1.72 & 1.62 & 37.27 & 9.31 & .68 & .80 \\
\hline & $\mathrm{MBSR}+\mathrm{AAT}$ & 43.30 & 6.83 & -1.08 & .64 & 41.10 & 5.71 & -1.10 & -.45 \\
\hline & Total & 44.00 & 10.37 & 2.69 & 1.68 & 39.10 & 7.86 & -.06 & .20 \\
\hline \multirow[t]{3}{*}{ Session 3} & MBSR & 39.91 & 8.89 & .60 & .14 & 37.45 & 8.61 & -.18 & .33 \\
\hline & $\mathrm{MBSR}+\mathrm{AAT}$ & 44.10 & 10.75 & -1.16 & .34 & 39.10 & 8.86 & .67 & 1.07 \\
\hline & Total & 41.90 & 9.81 & -.39 & .35 & 38.24 & 8.55 & .03 & .64 \\
\hline \multirow[t]{3}{*}{ Session 4} & MBSR & 41.09 & 10.95 & .24 & .46 & 37.18 & 7.04 & .55 & .04 \\
\hline & $\mathrm{MBSR}+\mathrm{AAT}$ & 39.10 & 9.37 & -1.72 & -.57 & 35.90 & 5.72 & -1.38 & -.11 \\
\hline & Total & 40.14 & 10.03 & -.29 & .14 & 36.57 & 6.32 & -.11 & .06 \\
\hline \multirow[t]{3}{*}{ Session 5} & MBSR & 43.27 & 12.69 & -.13 & .44 & 36.91 & 10.46 & -.12 & .83 \\
\hline & $\mathrm{MBSR}+\mathrm{AAT}$ & 38.80 & 8.46 & .71 & .66 & 36.30 & 5.38 & -.77 & .56 \\
\hline & Total & 41.14 & 10.86 & .33 & .69 & 36.62 & 8.24 & .71 & .90 \\
\hline \multirow[t]{3}{*}{ Session 6} & MBSR & 40.18 & 13.31 & -1.46 & .02 & 35.36 & 7.06 & -.38 & -.36 \\
\hline & $\mathrm{MBSR}+\mathrm{AAT}$ & 39.10 & 12.07 & -.86 & .11 & 37.60 & 6.90 & 2.90 & .84 \\
\hline & Total & 39.67 & 12.43 & -1.23 & .07 & 36.43 & 6.90 & .87 & .14 \\
\hline
\end{tabular}


Table C4

Descriptive Statistics for the TMS

\begin{tabular}{|c|c|c|c|c|c|c|c|c|c|}
\hline \multirow[b]{2}{*}{ TMS } & & \multicolumn{4}{|c|}{ Curiosity scale } & \multicolumn{4}{|c|}{ Decentering scale } \\
\hline & & Mean & $S D$ & Kurtosis & Skewness & Mean & $S D$ & Kurtosis & Skewness \\
\hline \multirow[t]{3}{*}{ Session 1} & MBSR & 14.00 & 4.36 & .41 & -1.21 & 15.09 & 5.67 & .12 & .33 \\
\hline & $\mathrm{MBSR}+\mathrm{AAT}$ & 14.09 & 5.65 & .76 & -1.25 & 13.90 & 4.86 & -.44 & -.87 \\
\hline & Total & 14.43 & 4.91 & .19 & -1.07 & 14.52 & 5.20 & .00 & -.03 \\
\hline \multirow[t]{3}{*}{ Session 2} & MBSR & 16.64 & 2.58 & 3.47 & 1.43 & 18.82 & 3.40 & .44 & -.82 \\
\hline & $\mathrm{MBSR}+\mathrm{AAT}$ & 16.50 & 5.64 & -.43 & -.43 & 17.00 & 4.16 & -1.67 & .10 \\
\hline & Total & 16.57 & 4.20 & .87 & -.30 & 17.95 & 3.80 & -1.15 & -.36 \\
\hline \multirow[t]{3}{*}{ Session 3} & MBSR & 17.00 & 2.06 & .74 & -.77 & 18.56 & 3.36 & -1.07 & -.06 \\
\hline & $\mathrm{MBSR}+\mathrm{AAT}$ & 17.33 & 4.77 & .71 & -.76 & 17.44 & 4.61 & -.83 & .09 \\
\hline & Total & 17.17 & 3.57 & 1.70 & -.70 & 18.00 & 3.96 & -.76 & -.10 \\
\hline \multirow[t]{3}{*}{ Session 4} & MBSR & 16.73 & 3.74 & 2.05 & -1.17 & 19.18 & 5.12 & 2.35 & -1.46 \\
\hline & $\mathrm{MBSR}+\mathrm{AAT}$ & 19.10 & 4.12 & -.82 & -.41 & 19.10 & 4.53 & -.08 & -.88 \\
\hline & Total & 17.86 & 4.02 & .41 & -.52 & 19.14 & 4.73 & .88 & -1.14 \\
\hline \multirow[t]{3}{*}{ Session 5} & MBSR & 18.36 & 2.54 & -.17 & .53 & 20.27 & 4.43 & -.99 & -.29 \\
\hline & $\mathrm{MBSR}+\mathrm{AAT}$ & 20.20 & 2.78 & -1.47 & -.10 & 21.80 & 3.65 & -1.08 & .15 \\
\hline & Total & 19.24 & 2.76 & -1.07 & .23 & 21.00 & 4.05 & -.71 & -.25 \\
\hline \multirow[t]{3}{*}{ Session 6} & MBSR & 24.36 & 2.84 & 1.62 & -.80 & 27.18 & 3.40 & 3.66 & -1.01 \\
\hline & $\mathrm{MBSR}+\mathrm{AAT}$ & 24.10 & 5.90 & 1.64 & -1.24 & 26.20 & 6.29 & 2.02 & -1.22 \\
\hline & Total & 24.24 & 4.44 & 2.79 & -1.31 & 26.71 & 4.88 & 3.14 & -1.38 \\
\hline
\end{tabular}




\section{Table C5}

Descriptive Statistics for Session Engagement Questionnaire

\begin{tabular}{|c|c|c|c|c|c|}
\hline Session & & Mean & $S D$ & Kurtosis & Skewness \\
\hline \multicolumn{6}{|l|}{ Session 1} \\
\hline \multirow[t]{3}{*}{ Mindful } & MBSR & 5.36 & 2.38 & -1.44 & .18 \\
\hline & $\mathrm{MBSR}+\mathrm{AAT}$ & 6.10 & 1.73 & -1.89 & -.19 \\
\hline & Total & 5.71 & 2.08 & -1.32 & -.10 \\
\hline \multirow[t]{3}{*}{ Engaged } & MBSR & 7.45 & 1.57 & -.38 & -.18 \\
\hline & $\mathrm{MBSR}+\mathrm{AAT}$ & 7.40 & 1.43 & .34 & .25 \\
\hline & Total & 7.43 & 1.47 & -.39 & -.00 \\
\hline \multirow[t]{3}{*}{ Looking forward } & MBSR & 9.00 & 1.10 & -1.11 & -.56 \\
\hline & $\mathrm{MBSR}+\mathrm{AAT}$ & 9.30 & .82 & -1.04 & .71 \\
\hline & Total & 9.14 & .96 & -.77 & -.68 \\
\hline \multicolumn{6}{|l|}{ Session 2} \\
\hline \multirow[t]{3}{*}{ Mindful } & MBSR & 7.45 & 1.13 & 1.66 & .90 \\
\hline & $\mathrm{MBSR}+\mathrm{AAT}$ & 6.90 & 1.79 & 1.38 & -1.26 \\
\hline & Total & 7.19 & 1.47 & 2.47 & -.99 \\
\hline \multirow[t]{3}{*}{ Engaged } & MBSR & 8.18 & 1.54 & .70 & -.97 \\
\hline & $\mathrm{MBSR}+\mathrm{AAT}$ & 8.00 & 2.16 & 2.58 & -1.49 \\
\hline & Total & 8.10 & 1.81 & 1.94 & -1.32 \\
\hline \multirow[t]{3}{*}{ Looking forward } & MBSR & 9.18 & .87 & -1.62 & -.41 \\
\hline & $\mathrm{MBSR}+\mathrm{AAT}$ & 9.20 & 1.03 & .95 & -1.24 \\
\hline & Total & 9.19 & .93 & -.29 & -.83 \\
\hline \multirow[t]{3}{*}{ Times practiced } & MBSR & 6.00 & 4.34 & .55 & 1.01 \\
\hline & $\mathrm{MBSR}+\mathrm{AAT}$ & 5.10 & 2.96 & .01 & .71 \\
\hline & Total & 5.57 & 3.68 & .84 & 1.04 \\
\hline \multicolumn{6}{|l|}{ Session 3} \\
\hline \multirow[t]{3}{*}{ Mindful } & MBSR & 7.18 & 1.17 & -.29 & -.42 \\
\hline & $\mathrm{MBSR}+\mathrm{AAT}$ & 6.80 & 1.55 & -1.91 & -.04 \\
\hline & Total & 7.00 & 1.34 & -1.26 & -.28 \\
\hline \multirow[t]{4}{*}{ Engaged } & MBSR & 8.00 & 1.18 & -.61 & .00 \\
\hline & $\mathrm{MBSR}+\mathrm{AAT}$ & 8.00 & 1.41 & -1.39 & -.30 \\
\hline & Total & 8.00 & 1.27 & -1.08 & -.16 \\
\hline & & & & \multicolumn{2}{|c|}{ (table continues) } \\
\hline
\end{tabular}




\begin{tabular}{|c|c|c|c|c|c|}
\hline Session & & Mean & $S D$ & Kurtosis & Skewness \\
\hline \multirow[t]{3}{*}{ Looking forward } & MBSR & 9.00 & 1.16 & .08 & -1.08 \\
\hline & $\mathrm{MBSR}+\mathrm{AAT}$ & 9.30 & 1.25 & 6.34 & -2.41 \\
\hline & Total & 9.15 & 1.18 & 1.88 & -1.59 \\
\hline \multirow[t]{3}{*}{ Times practiced } & MBSR & 5.91 & 3.81 & -1.01 & .52 \\
\hline & $\mathrm{MBSR}+\mathrm{AAT}$ & 5.10 & 4.07 & 3.72 & 1.79 \\
\hline & Total & 5.52 & 3.86 & .44 & 1.05 \\
\hline \multicolumn{6}{|l|}{ Session 4} \\
\hline \multirow[t]{3}{*}{ Mindful } & MBSR & 7.27 & 1.49 & 1.21 & -.79 \\
\hline & $\mathrm{MBSR}+\mathrm{AAT}$ & 8.00 & 1.05 & -.45 & .71 \\
\hline & Total & 7.62 & 1.32 & 1.58 & -.64 \\
\hline \multirow[t]{3}{*}{ Engaged } & MBSR & 8.00 & 1.84 & 6.08 & -2.22 \\
\hline & $\mathrm{MBSR}+\mathrm{AAT}$ & 9.10 & .99 & .91 & -1.09 \\
\hline & Total & 8.52 & 1.57 & 7.26 & -2.28 \\
\hline \multirow[t]{3}{*}{ Looking forward } & MBSR & 8.91 & 1.14 & -1.63 & -.29 \\
\hline & $\mathrm{MBSR}+\mathrm{AAT}$ & 9.40 & .97 & 4.19 & -1.96 \\
\hline & Total & 9.14 & 1.06 & -.60 & -.86 \\
\hline \multirow[t]{3}{*}{ Times practiced } & MBSR & 5.18 & 3.40 & 4.64 & 1.86 \\
\hline & $\mathrm{MBSR}+\mathrm{AAT}$ & 7.10 & 5.34 & .81 & 1.38 \\
\hline & Total & 6.10 & 4.43 & 2.07 & 1.64 \\
\hline \multicolumn{6}{|l|}{ Session 5} \\
\hline \multirow[t]{3}{*}{ Mindful } & MBSR & 8.36 & 1.21 & .13 & -.45 \\
\hline & $\mathrm{MBSR}+\mathrm{AAT}$ & 8.40 & .84 & .37 & .39 \\
\hline & Total & 8.38 & 1.02 & .25 & -.26 \\
\hline \multirow[t]{3}{*}{ Engaged } & MBSR & 8.64 & 1.12 & -1.23 & -.16 \\
\hline & $\mathrm{MBSR}+\mathrm{AAT}$ & 8.70 & .95 & -.35 & -.23 \\
\hline & Total & 8.67 & 1.02 & -.96 & -.19 \\
\hline \multirow[t]{3}{*}{ Looking forward } & MBSR & 9.45 & .82 & -.25 & -1.15 \\
\hline & $\mathrm{MBSR}+\mathrm{AAT}$ & 9.50 & .97 & 5.36 & -2.27 \\
\hline & Total & 9.48 & .87 & 2.12 & -1.67 \\
\hline \multirow[t]{4}{*}{ Times practiced } & MBSR & 7.18 & 5.17 & -1.52 & .68 \\
\hline & $\mathrm{MBSR}+\mathrm{AAT}$ & 5.00 & 3.56 & -1.26 & .24 \\
\hline & Total & 6.14 & 4.51 & -.55 & .78 \\
\hline & & & & \multicolumn{2}{|c|}{ (table continues) } \\
\hline
\end{tabular}




\begin{tabular}{llcccc}
\hline Session & & Mean & SD & Kurtosis & Skewness \\
\hline Session 6 & & & & & \\
\multicolumn{1}{c}{ Mindful } & MBSR & 8.09 & 1.04 & -.26 & -.86 \\
& MBSR + AAT & 8.30 & 1.49 & -1.62 & -.14 \\
& Total & 8.19 & 1.25 & -.98 & -.23 \\
Engaged & MBSR & 8.73 & 1.35 & .01 & -.89 \\
& MBSR + AAT & 8.90 & 1.37 & -1.47 & -.75 \\
\multirow{2}{*}{ Times practiced } & Total & 8.81 & 1.33 & -.81 & -.75 \\
& MBSR & 4.64 & 2.38 & 1.24 & 1.24 \\
& MBSR + AAT & 6.30 & 4.11 & -1.67 & -.09 \\
& Total & 5.43 & 3.34 & -.92 & .53 \\
\hline
\end{tabular}




\section{Table C6}

\section{Descriptive Statistics for Client Satisfaction Questionnaire}

\begin{tabular}{|c|c|c|c|c|c|}
\hline Variable & & Mean & $S D$ & Kurtosis & Skewness \\
\hline \multirow[t]{3}{*}{ Enjoy the training } & MBSR & 4.00 & .00 & & \\
\hline & $\mathrm{MBSR}+\mathrm{AAT}$ & 4.00 & .00 & & \\
\hline & Total & 4.00 & .00 & & \\
\hline \multirow[t]{3}{*}{ Effective therapist } & MBSR & 3.82 & .41 & 2.04 & -1.92 \\
\hline & $\mathrm{MBSR}+\mathrm{AAT}$ & 4.00 & .00 & & \\
\hline & Total & 3.90 & .30 & 7.56 & -2.98 \\
\hline \multirow[t]{3}{*}{ Use the skills } & MBSR & 3.73 & .47 & -.76 & -1.19 \\
\hline & $\mathrm{MBSR}+\mathrm{AAT}$ & 3.90 & .32 & 10.00 & -3.16 \\
\hline & Total & 3.81 & .40 & .98 & -1.70 \\
\hline \multirow[t]{3}{*}{ Recommend the training } & MBSR & 3.64 & .51 & -1.96 & -.66 \\
\hline & $\mathrm{MBSR}+\mathrm{AAT}$ & 3.90 & .32 & 10.00 & -3.16 \\
\hline & Total & 3.76 & .44 & -.28 & -1.33 \\
\hline \multirow[t]{3}{*}{ Interaction with therapist } & MBSR & 3.91 & .30 & 11.00 & -3.32 \\
\hline & $\mathrm{MBSR}+\mathrm{AAT}$ & 3.90 & .32 & 10.00 & -3.16 \\
\hline & Total & 3.90 & .30 & 7.56 & -2.98 \\
\hline \multirow[t]{3}{*}{ Deal more effectively } & MBSR & 3.27 & .47 & -.76 & 1.19 \\
\hline & $\mathrm{MBSR}+\mathrm{AAT}$ & 3.40 & .70 & -.15 & -.78 \\
\hline & Total & 3.33 & .58 & -.54 & -.13 \\
\hline \multirow[t]{3}{*}{ Satisfied with training } & MBSR & 3.73 & .47 & -.76 & -1.19 \\
\hline & $\mathrm{MBSR}+\mathrm{AAT}$ & 3.60 & .52 & -2.28 & -.48 \\
\hline & Total & 3.67 & .48 & -1.58 & -.76 \\
\hline \multirow[t]{3}{*}{ Participate in future } & MBSR & 3.45 & .52 & -2.44 & .21 \\
\hline & $\mathrm{MBSR}+\mathrm{AAT}$ & 3.80 & .42 & 1.41 & -1.78 \\
\hline & Total & 3.62 & .50 & -1.91 & -.53 \\
\hline Complete with no dog & $\mathrm{MBSR}+\mathrm{AAT}$ & 3.50 & .53 & -2.57 & .00 \\
\hline Dog make it more helpful/enjoyable & $\mathrm{MBSR}+\mathrm{AAT}$ & 3.80 & .42 & 1.41 & -1.78 \\
\hline
\end{tabular}




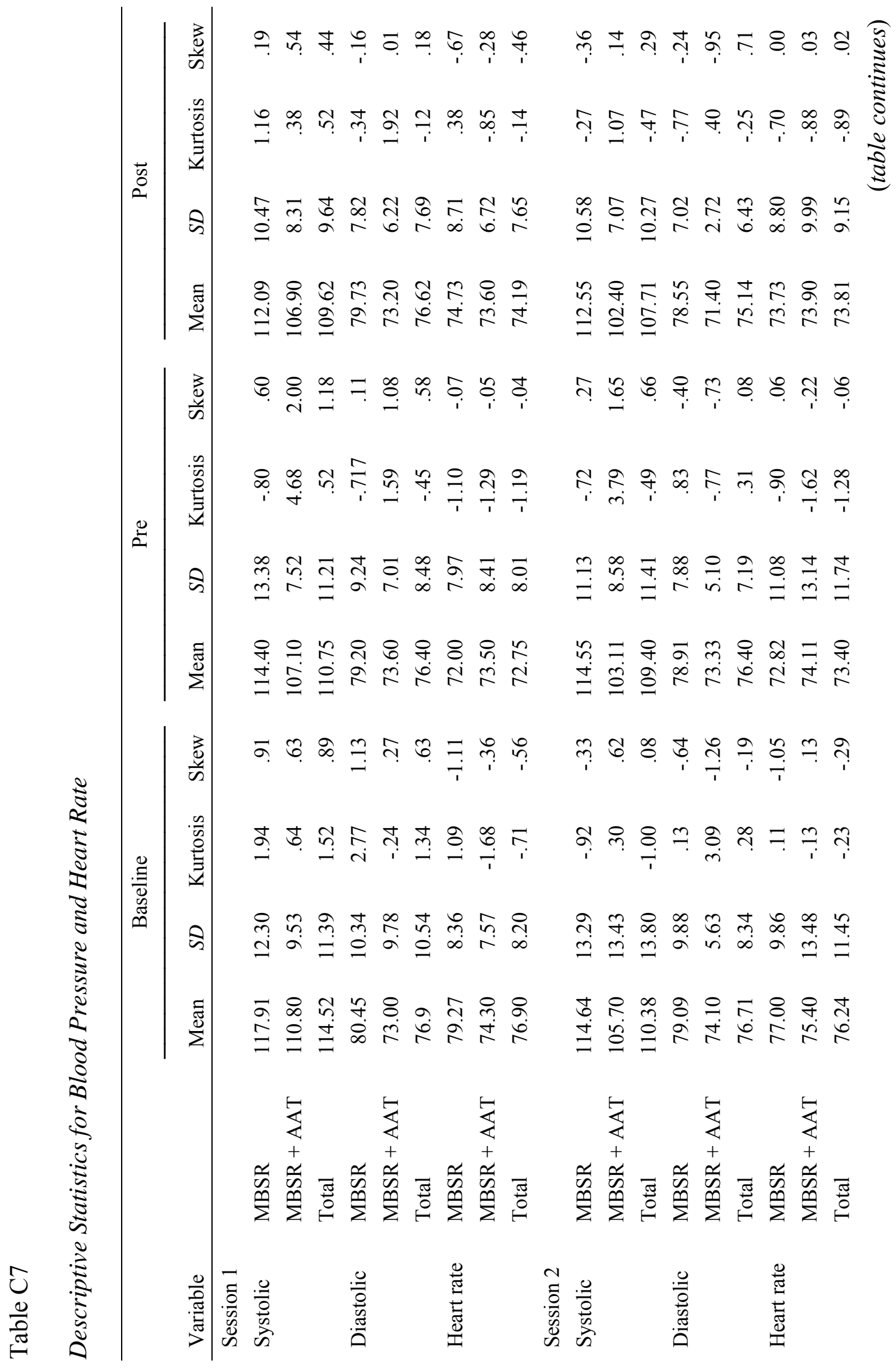




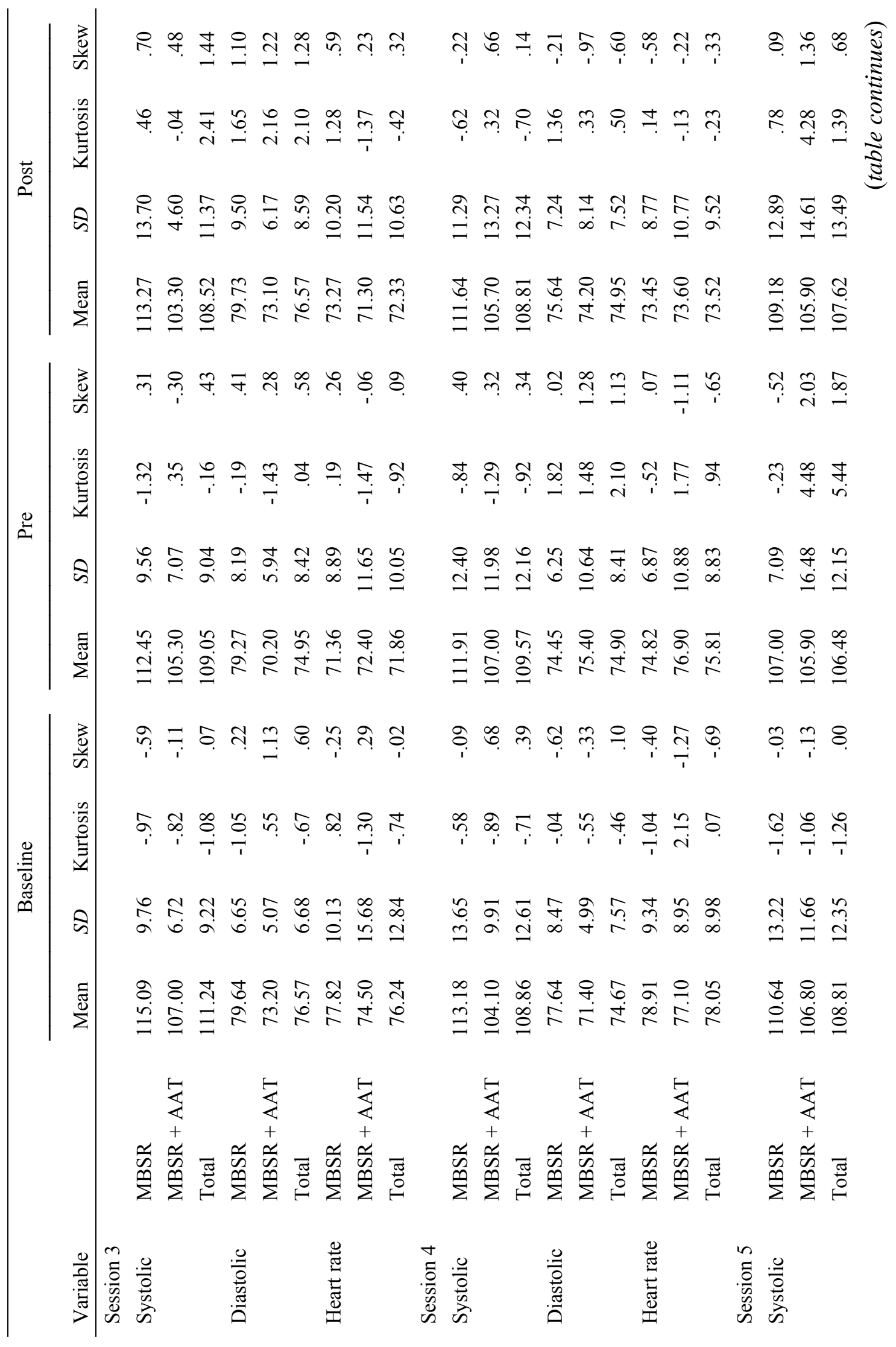




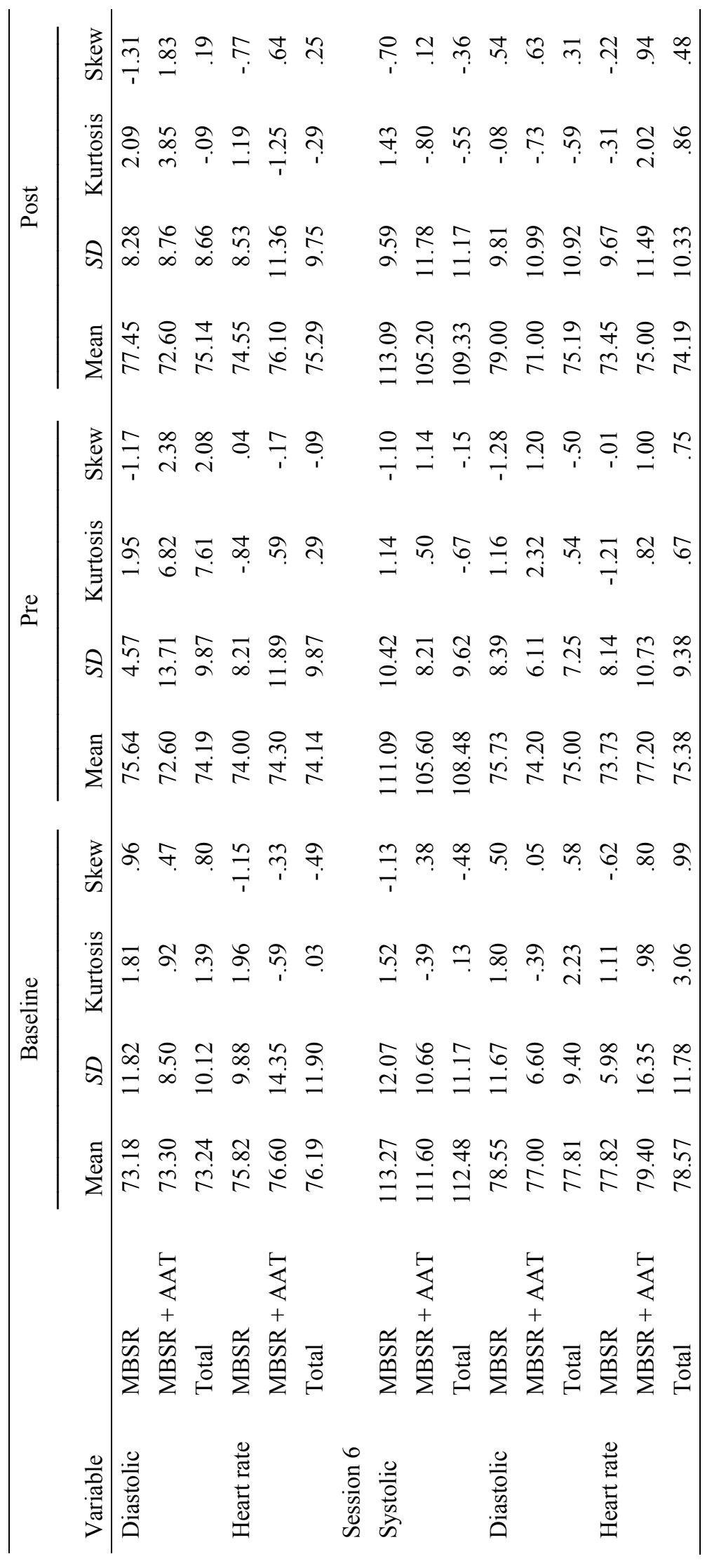




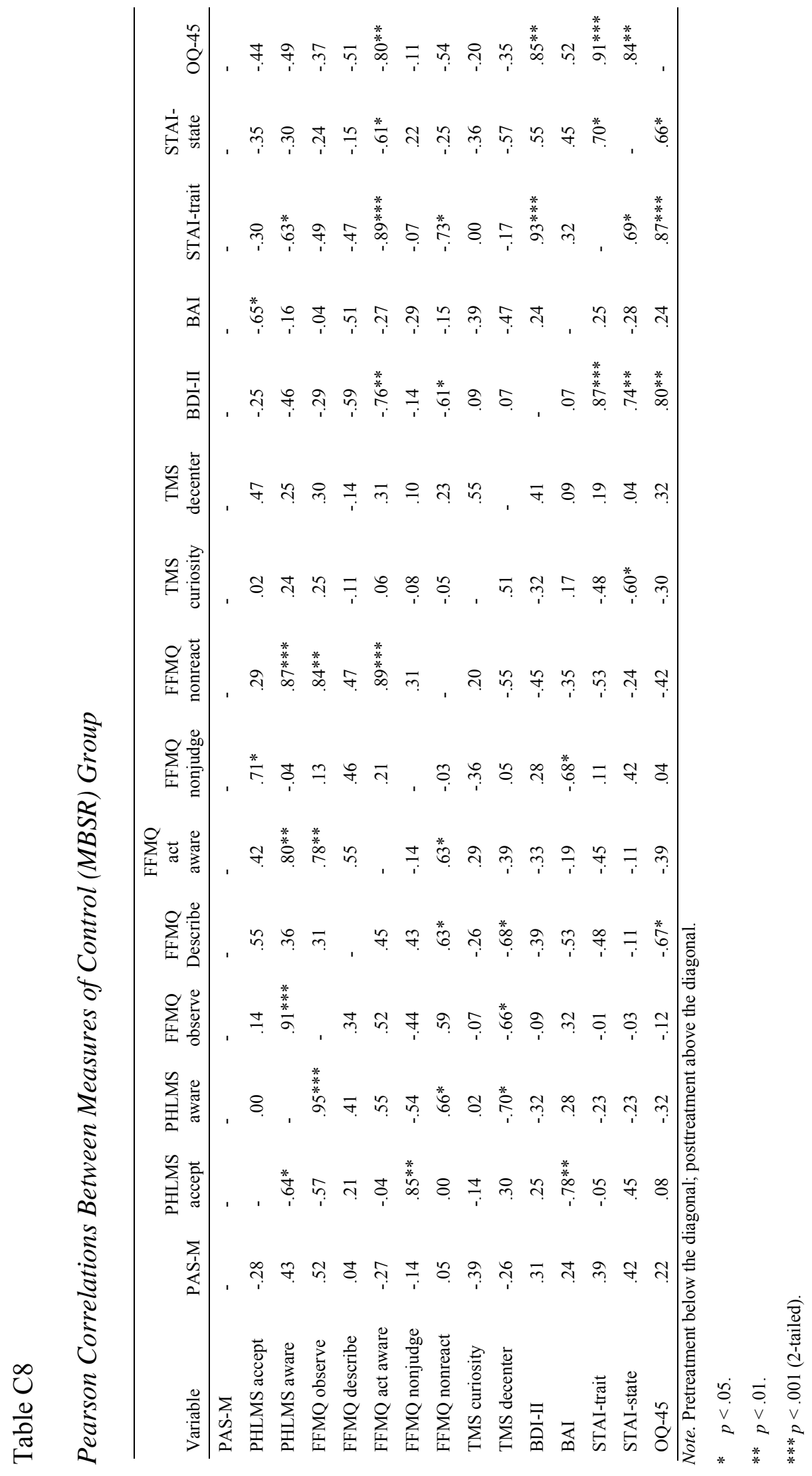




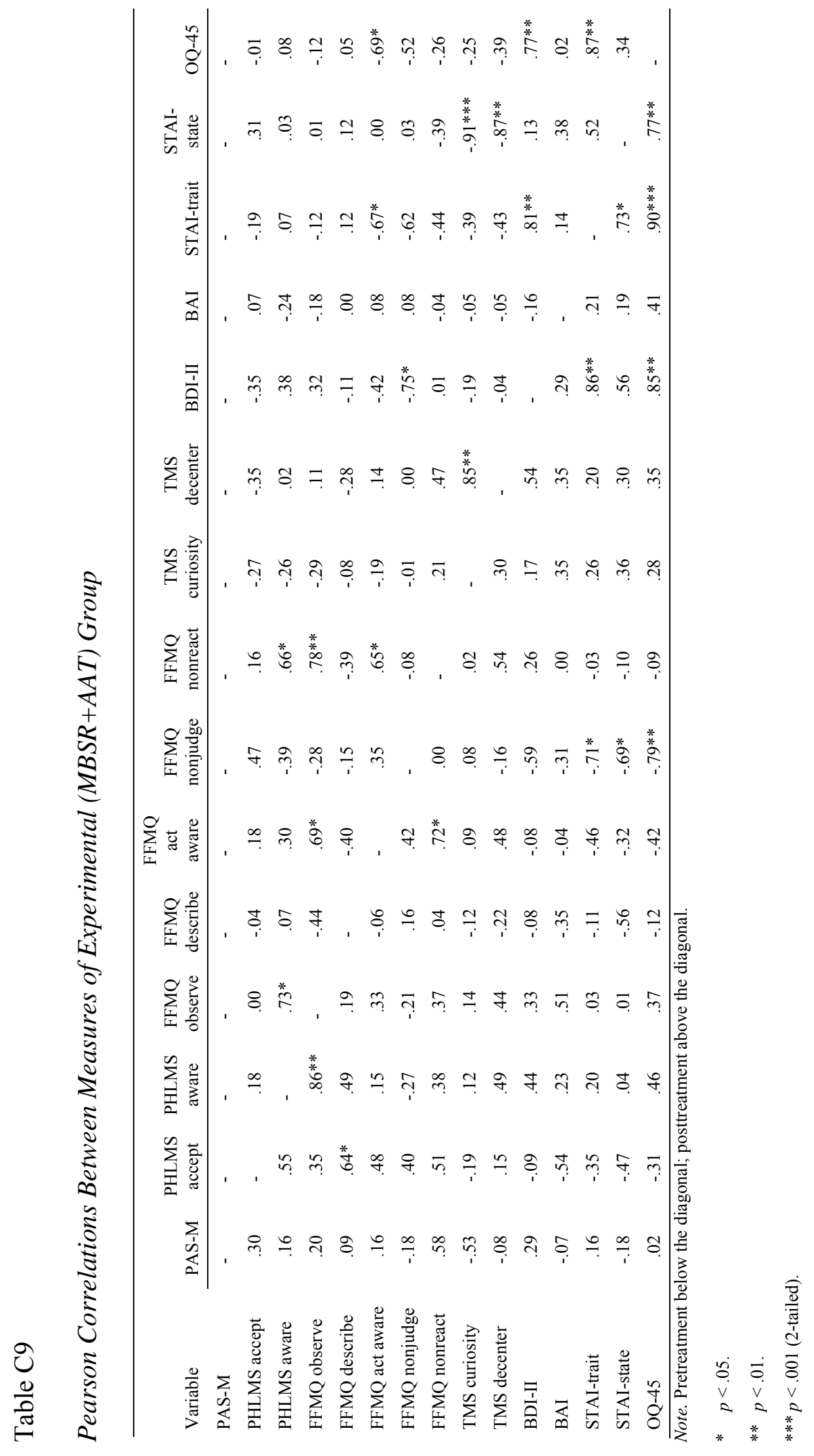




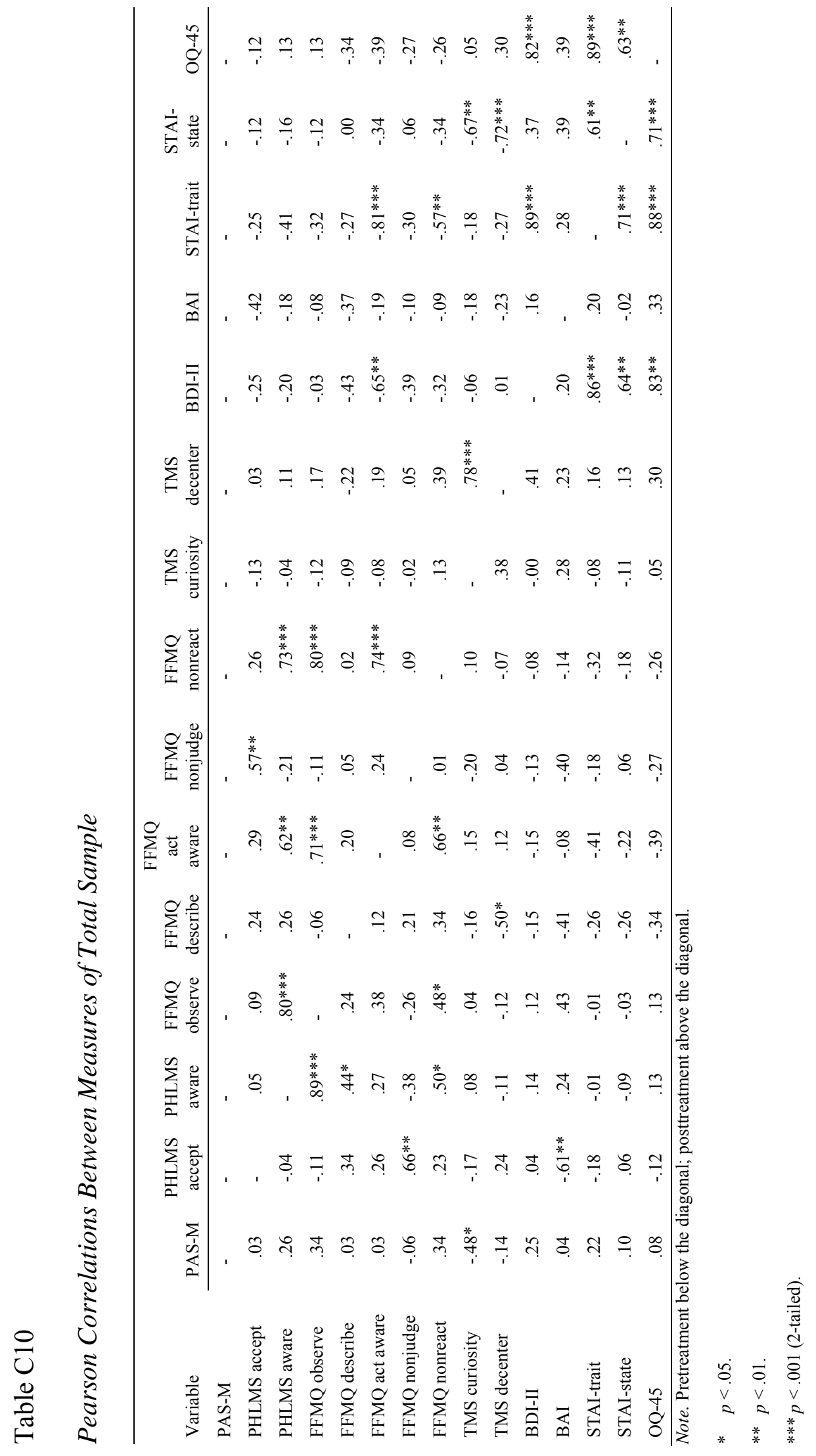




\section{CURRICULUM VITAE}

\section{COURTNEY L. HENRY}

532 East 200 South

Logan, UT 84321

(856) 313-7793

courtney.henry@aggiemail.usu.edu

\section{EDUCATION}

Ph.D. Combined Clinical/Counseling/School Psychology (APA accredited)

2013 Utah State University, Logan, UT

Dissertation: The psychological and physiological effects of using a therapy dog in mindfulness training. Chair: Susan Crowley, Ph.D.

M.S. Human Development

2006 University of Rochester, Rochester, NY

Thesis: Emotional security theory: Children's dimensional responses to interparental conflict. Chair: Paul Stein, Ph.D.

B.A. Brain and Cognitive Sciences; Psychology

2003 University of Rochester, Rochester, NY

\section{WORK EXPERIENCE}

Psychology Intern (08/12 - current; 40 hours weekly)

APA Accredited Internship, USU Counseling and Psychological Services Center, Logan, UT

$\underline{\text { Clinical }}$

- Provided individual and couples psychotherapy to college students.

- Provided brief consult counseling, intake assessment, and crisis intervention services.

- Co-led weekly process group entitled Understanding Self and Others Group for students with a variety of issues including anxiety, depression, and relationship issues.

- Developed and co-led a weekly support group entitled Pet Loss Support Group for students and community members grieving the loss of an animal.

- Co-led a semester-long psychoeducational group entitled Skills Training Group, teaching students a variety of skills from Dialectical Behavior Therapy.

$\underline{\text { Assessment }}$

- Conducted full-battery learning disability, ADHD, and neuropsychological evaluations.

- Scored and interpreted various intelligence, academic achievement, cognitive, personality, and symptom assessment instruments.

- Wrote comprehensive evaluation reports and provided recommendations.

$\underline{\text { Supervision }}$

- Supervised a doctoral student during her practicum for one semester. 
- Supervised an undergraduate Reach Peer trained to teach various skills for two semesters.

\section{Outreach and Consultation}

- Provided psychological consultation services to the Access and Diversity Center, including weekly animal-assisted visitation for staff and students.

- Created and conducted a workshop for students entitled Take a Walk on the Wild Side: The Mental Health Benefits of Nature.

- Developed and presented a workshop to counseling center staff entitled Developmental Considerations for Clients with Autism.

- Participated in other outreach, including:

- Provided an interview about animal-assisted therapy and counseling services for the campus newspaper.

- Provided an interview about mindfulness and animal-assisted therapy for the campus television channel.

- Provided interpretation of anxiety and depression measures and consultation to students attending USU CAPS anxiety and depression screening days.

- Provided animal-assisted visitation and consultation on stress management and self-care to students attending the USU Stress Bust during final exam weeks.

- Presented off-campus about stress management to parents of children with Autism.

- Presented about counseling services at student orientation for International Student Services.

Training

- Participated in weekly and bi-weekly training seminars, including : Assessment, Supervision of Supervision, Consultation and Outreach, Specialty Seminar, Case Presentation, Group Therapy, and Multicultural Seminar, as well as other professional development activities.

- Received weekly individual supervisions, including both clinical and professional development.

- Attended staff meetings and administrative meetings.

- Participated in agency and self-sponsored continuing education workshops.

Graduate Assistant Student Therapist (08/11 - 05/12; 20 hours weekly)

USU Counseling and Psychological Services, Logan, Utah

Supervisor: Dave Bush, Ph.D.

- Provided consults, intakes, individual, and group therapy to students.

- Provided animal-assisted therapy to individual, group, and outreach clients.

- Attended regular staff meetings and professional development seminars.

- Supervised an undergraduate Reach Peer trained to teach various skills for two semesters.

- Provided outreach services, including:

- Provided animal-assisted visitation for staff and students during a suicide debriefing.

- Led workshops about sleep hygiene for college students, faculty, and staff.

- Provided interpretation of anxiety measures and consultation to students attending the USU CAPS anxiety screening day.

- Presented information about counseling services to residential assistants.

Student Therapist $(05 / 10-08 / 12)$

USU Community Psychology Clinic, Logan, Utah

Supervisors: Susan Crowley, Ph.D.; Gretchen Gimpel-Peacock, Ph.D.; Scott DeBerard, Ph.D.; Carolyn Barcus, Ed.D. 
- Provided individual therapy, couples therapy, and parent training to community members.

- Provided animal-assisted therapy in individual sessions.

- Maintained clinical notes and scheduling.

Student Group Co-therapist (06/10 - 06/12)

USU Community Psychology Clinic, Logan, Utah

Supervisors: Carolyn Barcus, Ed.D.; Susan Crowley, Ph.D.

- Co-led an on-going process group for adult women survivors of severe childhood sexual abuse.

Applied Behavior Analysis Support Staff (08/06 - 08/08; 40 hours weekly)

Burlington County Special Services School District, Mount Holly, NJ

Supervisor: Adell Valasek, M.SpEd., BCBA.

- Developed and implemented instructional programs, behavior modification plans, and prompting strategies within school and home settings for children diagnosed with Autism Spectrum Disorder.

- Trained in discrete trial, applied verbal behavior, and daily living skill services, as well as Nonviolent Crisis Intervention.

- Attended clinic and individualized education plan meetings.

- Collected and recorded behavioral data.

- Maintained ethical and collaborative relationships with families and school district employees, including school psychologists, occupational and speech therapists, social workers, and teachers.

\section{CLINICAL PRACTICA}

\section{Practicum Student Therapist $(05 / 11-05 / 12)$}

Practicum in School/Child Clinical Psychology

Up to 3, Logan, Utah

Supervisor: Gretchen Gimpel Peacock, Ph.D.

- Completed behavioral evaluations and provided in-home services for children ages 0 to 3 years and their families.

- Conducted behavioral interventions and provided parent training.

- Maintained scheduling and clinical notes in accordance with government regulations.

Practicum Student Therapist $(05 / 11$ - 08/11)

Advanced Practicum in Counseling/Clinical Psychology

USU Counseling and Psychological Services, Logan, Utah

Supervisors: Dave Bush, Ph.D.; AJ Grovert, M.S.

- Provided intakes and individual therapy to undergraduate and graduate students.

- Provided animal-assisted therapy and visitation to students, faculty, and staff.

- Attended staffing and administrative meetings.

- Provided outreach services, including workshops about sleep hygiene.

Practicum Student Therapist (08/10 - 05/11)

Practicum in Counseling/Clinical Psychology

USU Counseling and Psychological Services, Logan, Utah

Supervisors: Thomas Berry, Ph.D.; AJ Grovert, M.S. 
- Provided intakes and individual therapy to undergraduate and graduate students.

- Maintained scheduling and clinical notes in Titanium.

- Provided outreach services to college students, faculty, and staff, including:

- Led workshops about sleep hygiene.

- Provided interpretation of anxiety and depression measures and consultation to students attending the USU CAPS anxiety and depression screening days.

- Provided animal-assisted visitation and consultation on stress management and self-care to students attending the USU Stress Bust during fall and spring final exam weeks.

- Co-led a workshop on self-care and informal mindfulness practice at USU Brigham City campus.

Practicum Student Therapist $(08 / 09$ - 05/10)

Integrative Practicum with Adults, Adolescents, and Children

USU Community Psychology Clinic, Logan, Utah

Supervisors: Susan Crowley, Ph.D.; Kyle Hancock, Ph.D.

- Performed intakes and individual therapy for children, adolescents, and adults.

- Maintained clinical notes and scheduling.

- Conducted and interpreted assessments for learning disorders and ADHD.

- Wrote evaluation reports and suggested student accommodations for the USU Disability Resource Center.

\section{TEACHING EXPERIENCE}

Instructor (Spring 09 - Summer 11; 20 hours weekly)

Department of Psychology, Utah State University, Logan, UT

Supervisor: Gretchen Gimpel-Peacock, Ph.D.

Psy 4950: Undergraduate Apprenticeship (Fall 10, Spring 11)

Psy 3120: Abuse and Neglect (Fall 09)

Psy 1100: Developmental Psychology: Infancy and Childhood - Broadcast (Summers 09 \& 11)

Psy 1100: Developmental Psychology: Infancy and Childhood (Springs 09 \& 10)

- Developed curriculum, lectured, and managed Blackboard and online resources.

- Created examinations and assessments, graded papers, and provided written feedback.

- Held office hours, responded to students' questions and concerns, and worked cooperatively with teaching assistants, faculty, and various community organizations.

Teaching Assistant (Fall 08 - Spring 11; 10 hours weekly)

Department of Psychology Utah State University, Logan, UT

Supervisors: Frank Ascione, Ph.D.; Susan Crowley, Ph.D.; Jessica Gundy, M.S.; Spencer

Richards, B.A.

Psy 7350: Integrative Practicum with Adults, Adolescents, and Children (Fall 10, Spring 11)

Psy 2800: Psychological Statistics (Summer 10)

Psy 2100: Developmental Psychology: Adolescence (Spring 09)

Psy 1100: Developmental Psychology: Infancy and Childhood (Fall 08)

Psy 3120: Abuse and Neglect (Fall 08)

- Held office hours, responded to students' questions and concerns, created and led review sessions, and guest lectured.

- Proctored and scored exams as well as graded papers.

- Evaluated the administration of intellectual and academic achievement assessments for 
doctoral psychology students.

\section{Guest Lecturer}

Department of Psychology, Utah State University, Logan, UT

- Lectured on Autism Spectrum Disorder for Psy 1010: Introductory Psychology (11/08)

- Lectured on neurobiology for Psy 1010: Introductory Psychology (09/08)

\section{RESEARCH EXPERIENCE}

Research Co-Investigator (07/10 - 09/11)

Department of Psychology, Utah State University, Logan, UT

- Developed a study with Clint Field, Ph.D. and Jennifer Yardley, M.S. to assess the efficacy of mindfulness-based interventions for clinically diagnosed children and adolescents. Performed a literature review and manuscript writing.

Research Assistant (05/04 - 08/06; 40 hours weekly) Mount Hope Family Center, University of Rochester, Rochester, NY Supervisor: Patrick Davies, Ph.D.

- Collected data for Project FUTURE, a longitudinal NIMH-funded study to examine the effects of domestic violence on child development, led by investigators Patrick Davies, Ph.D. and Dante Cicchetti, Ph.D.

- Developed protocols, recruited and screened subjects, and acted as experimenter, working with at-risk toddlers and their mothers.

- Collected physiological data, including cortisol levels and vagal tones.

- Administered child mental scales, adult interviews, and tasks to assess attachment and temperament.

- Successfully completed the Ethical Principles in Research Program.

Project Coordinator (05/03 - 05/04; 40 hours weekly) Department of Psychology, University of Rochester, Rochester, NY Supervisor: Patrick Davies, Ph.D.

- Managed the Me and My Family Project, a multisite research project to longitudinally study the effects of interparental discord on the social and emotional adjustment of children.

- Coordinated with staff and faculty from Notre Dame and organized the laboratory.

- Interviewed, supervised, and evaluated undergraduate research assistants.

- Maintained large databases in Access and Excel, as well as analyzed data in SPSS.

Undergraduate Research Assistant (08/00 - 05/03; 20 hours weekly) Department of Psychology, University of Rochester, Rochester, NY Supervisor: Patrick Davies, Ph.D.

- Served in the role of experimenter, interviewing and working with community families in a laboratory setting, as well as monitored subject payment and operated video camera equipment.

- Successfully completed the Human Subjects Protection Program.

- Completed self-report data coding and entry, requiring theoretical insight.

- Aided study coordinator with logistics of implementation for the Me and My Family Project. 


\section{PRESENTATIONS}

Nash, T., Kissel, S., MacFarlane, I. M., \& Henry, C. L. (2012, November). Utah State University's CAPS: A three year look at client satisfaction. Poster presented at the annual Utah University \& College Counseling Centers Conference, Park City, UT.

Henry, C. L., \& Crowley, S. L. (2011, October). The Use of Animal-Assisted Therapy in College Counseling Centers. Poster presented at the annual Utah University \& College Counseling Centers Conference, Park City, UT.

Henry, C. L., \& Crowley, S. L. (2011, August). The psychological and physiological effects of incorporating a therapy dog into mindfulness training: A case study. Poster presented at the annual conference of the International Society for Anthrozoology, Indianapolis, IN.

Henry, C. L., Yardley, J., \& Field, C. (2010, November). A review of empirical support for the use of mindfulness-based interventions with clinically diagnosed child and adolescent populations. Poster presented at the annual convention for the Association for Behavioral and Cognitive Therapies, San Francisco, CA.

Sturge-Apple, M., Davies, P., Cummings, E., \& Henry, C. (2005, April). Interdependencies among marital conflict, parental warmth and child internalizing symptoms: An examination of reciprocal effects. Poster presented at the Society for Research in Child Development Biennial Meeting, Atlanta, GA.

Sturge-Apple, M., Davies, P., Cummings, E., \& Henry, C. (2005, April). Marital withdrawal, parental emotional unavailability, and child adjustment difficulties: A process model. Poster presented at the Society for Research in Child Development Biennial Meeting, Atlanta, GA.

\section{ATTENDED WORKSHOPS AND TRAININGS}

01/13 USU Counseling and Psychological Services, Logan, UT

Breaking the Silence: Healing the Shame of Male Survivors of Sexual Abuse and Assault Jim Struve, LCSW, Half-day workshop

04/12 USU Counseling and Psychological Services, Logan, UT

The How, What, and Why of Happiness

Sonya Lyubomirsky, Ph.D.; Full-day workshop

10/11 Faces Conferences: The Arts of Mindfulness \& Counseling, Seattle, WA Mindfulness Skills \& Dialectical Behavior Therapy

Marsha Linehan, Ph.D.; Half-day workshop

Wedding Contemplative Psychology \& Western Psychology: Healing \& Transformation Jack Kornfield, Ph.D.; Half-day workshop

04/11 USU Counseling and Psychological Services, Logan, UT

Addictive Behaviors

Jason Kilmer, Ph.D.; Full-day workshop 
11/10 Association for Behavioral and Cognitive Therapies, San Francisco, CA Integrating DBT Skills into Clinical Practices: Emotion Regulation Marsha Linehan, Ph.D. and Kathryn Korslund, Ph.D.; Full-day training Case Conceptualization in ACT Daniel Moran, Ph.D. and Patricia Bach, Ph.D.; Half-day workshop Mindfulness and Values Work to Foster Behavioral Activation: An Acceptance and Commitment Therapy Perspective

Kelly Wilson, Ph.D. and Emily Sandoz, Ph.D.; Half-day workshop

11/10 Jefferson-Myrna Brind Center of Integrative Medicine, Philadelphia, PA Mindfulness-Based Stress Reduction Workshop for Professionals

Diane Reibel, Ph.D. and Donald McCown, M.S.S., M.A.M.S.; Full-day workshop

04/10 USU Counseling and Psychological Services, Logan, UT An Integrated Approach to Complex Psychological Trauma John Briere, Ph.D.; Full-day workshop

11/09 Utah State University, Logan, UT GLBT Allies on Campus Training Maure Smith; Half-day training

04/09 Utah Psychological Association \& Avalon Hills Residential Eating Disorder Program, Logan, UT Acceptance and Commitment Therapy Steven Hayes, Ph.D.; Two-day workshop

10/08 USU Department of Psychology, Logan, UT

ACT-based Multicultural Competence

Michael Twohig, Ph.D. and Melanie Domenech Rodriguez, Ph.D.; Half-day workshop

\section{HONORS AND AWARDS}

04/11 Elwin C. Nielsen Scholarship, \$700, Utah State University

11/10 Psychology Department Travel Award, \$300, Utah State University

11/10,10/11 Graduate Student Senate Travel Award, \$300, Utah State University

08/08 - 05/09 Vice Presidential Research Fellowship, Utah State University

08/01 - 05/03 Dean's List awards, University of Rochester

08/99 - 05/03 Rush Rhees Scholarship, University of Rochester

\section{PROFESSIONAL AFFILIATIONS}

American Psychological Association, Student Affiliate

Association for Behavioral and Cognitive Therapies, Student Affiliate

International Society for Anthrozoology, Student Affiliate

Psi Chi, National Honor Society in Psychology, University of Rochester

Society of Counseling Psychology, Student Affiliate

Utah Psychological Association, Student Affiliate 